MIT-CTP-3219, JLAB-THY-02-06

\title{
Moments of Nucleon Light Cone Quark Distributions Calculated in Full Lattice QCD
}

\author{
LHPC and SESAM Collaborations
}

D. Dolgov, R. Brower, S. Capitani*, P. Dreher, J. W. Negele, A. Pochinsky, D. B. Renner

Center for Theoretical Physics, Laboratory for Nuclear Science, Massachusetts Institute of Technology,

77 Massachusetts Avenue, Cambridge, Massachusetts 02139

\author{
N. Eicker, Th. Lippert, K. Schilling \\ Department of Physics, University of Wuppertal, D-42097 Wuppertal, Germany \\ R. G. Edwards \\ Jefferson Lab, 12000 Jefferson Avenue, MS 12H2, Newport News, Virginia 23606 \\ U. M. Heller \\ CSIT, Florida State University, Tallahassee, Florida 32306
}

(January 28, 2002)

\begin{abstract}
Moments of the quark density, helicity, and transversity distributions are calculated in unquenched lattice QCD. Calculations of proton matrix elements of operators corresponding to these moments through the operator product expansion have been performed on $16^{3} \times 32$ lattices for Wilson fermions at $\beta=5.6$ using configurations from the SESAM collaboration and at $\beta=5.5$ using configurations from SCRI. One-loop perturbative renormalization corrections are included. At quark masses accessible in present calculations, there is no statistically significant difference between quenched and full QCD results, indicating that the contributions of quark-antiquark excitations from the Dirac Sea are small. Close agreement between calculations with cooled configurations containing essentially only instantons and the full gluon configurations indicates that quark zero modes associated with instantons play
\end{abstract}

*present address DESY/Zeuthen 
a dominant role. Naive linear extrapolation of the full QCD calculation to the physical pion mass yields results inconsistent with experiment. Extrapolation to the chiral limit including the physics of the pion cloud can resolve this discrepancy and the requirements for a definitive chiral extrapolation are described.

11.15.Ha, 12.38.Gc, 13.60.Hb, 14.20.Dh

Typeset using REVTEX 


\section{INTRODUCTION}

The quest to understand the matter of which our universe is composed will remain fundamentally incomplete until we understand how the quark and gluon structure of the nucleon arises from QCD. The nucleon has many remarkable properties. Because of confinement, the quark-gluon structure of hadrons differs essentially from that of any other known composite systems. Gluons in QCD are essential dynamical degrees of freedom, unlike the boson fields in atoms and nuclei which may be subsumed into a two-body interaction thereby reducing these systems to purely fermionic degrees of freedom. Almost all of the mass and approximately half of the momentum and angular momentum of a nucleon arises from gluons. Indeed, even the net spin $1 / 2$ arises from a rich and complicated combination of orbital and intrinsic angular momentum of the quark and gluon fields. Since the usual analytic tools of theoretical physics have proven inadequate to solve nonperturbative QCD, the only known way to solve, rather than model, QCD is numerical solution of lattice field theory. Hence, the ultimate goal of this work is to use lattice QCD to understand the structure of the nucleon. This understanding has two important but distinct aspects.

One aspect of using lattice QCD to understand nucleon structure is the quantitative $a b$ initio calculation of experimental observables. Since the experimental discovery of quarks in the nucleon over a quarter of a century ago, there has been a huge investment internationally in using high energy scattering to measure the light cone distribution of quarks and gluons in the nucleon. As a result of several decades of experimental effort at SLAC, Fermilab, CERN, and DESY, we now have a detailed knowledge of the quark density and helicity distributions and of the gluon distribution [1 8]. In addition, major new experiments are being planned at these facilities as well as at Jefferson Lab and RHIC to map out the quark and gluon structure of the nucleon in even more detail. Now that the techniques of lattice field theory and computer technology have developed to the point that it will be possible to solve QCD with a precision comparable to experimental measurements, it is essential to complement this massive experimental investment with a commensurate theoretical effort in lattice QCD. Because deep inelastic lepton scattering measures correlation functions close to the light cone, structure functions are intrinsically Minkowski and cannot be calculated directly in lattice QCD. However, using the operator product expansion, it is possible to calculate their moments. In this work, we report the first calculations of these moments in full QCD [9]. As will be emphasized below, these present calculations are subject to significant limitations due to current computer resources.

The second aspect is understanding the basic mechanisms underlying nucleon structure - that is, how QCD actually works. Hence, we seek to use the lattice as a tool for insight as well as for numbers. There are several ways lattice calculations can provide insight. One is to calculate the overlap between a trial wave function and the exact nucleon wave function to explore the role of various degrees of freedom and variational parameters. Thus, this work will present a prototype variational calculation in which the rms radius of a trial function is varied. A second is to study the contributions of different classes of Feynman diagrams that correspond to separate lattice contributions to operators. An example is the class of connected diagrams considered in this work. Finally, since the lattice Monte Carlo calculation stochastically samples gluons distributed according to the QCD action, one can identify and study the dominant configurations. Hence, this work will study the role of 
instantons and their associated zero modes in calculating moments of structure functions.

There have been several calculations of moments of structure functions in recent years, including contributions of connected diagrams to low moments of the spin independent and longitudinal spin dependent structure functions in quenched lattice QCD [10 12,9], disconnected contributions for the axial and tensor charge [13], and the axial charge in full as well as quenched QCD [14]. Interestingly, in contrast to spectroscopy, there are significant discrepancies between these hadron structure calculations and experiment. Whereas quenched masses for light hadrons are typically accurate at the $5 \%$ level, the axial charge is typically $10-20 \%$ low [15] and the first moment of the spin averaged structure function is of the order of $50 \%$ high [10,11]. By the variational principle, we know that an error of order $\epsilon$ in the wave function only produces an error of order $\epsilon^{2}$ in the expectation value of the energy, or mass in this case, so it is consistent that these other observables should be much better diagnostics than masses of the errors in the lattice calculation of hadron structure. Hence, we need to identify and correct the source of these substantial discrepancies.

There are three major limitations in structure calculations imposed by limitations in computational resources. The first limitation is errors in approximating the continuum limit. The approach to the continuum limit has been studied systematically in quenched QCD by the QCDSF collaboration [10] using Wilson and clover improved actions and extrapolating in inverse coupling $\beta$. A particularly careful study was carried out for the axial charge which showed that even when the $\beta$ dependence is included, there remains a $10 \%$ discrepancy with experiment [15].

The second limitation is the quenched approximation, which ignores the contribution of dynamical quark-antiquark excitations in the Dirac sea. It is now known that whereas full QCD gives a good description of masses of hadrons containing strange quarks, quenching causes significant discrepancies in masses. Physically, this is consistent with the fact that in quenched QCD the coupling runs too fast, producing a potential that becomes too weak at the short distances probed by the relatively heavy strange quark. Although there is no simple argument suggesting the sign of the resulting discrepancy in other specific observables, it is important to see to what extent quenching causes the discrepancy in the axial charge and the first moment of structure functions. Thus, a primary goal of this work is to study the role of the quenched approximation. Although the effects of the lattice spacing and lattice volume are still significant potential sources of error, it is meaningful to compare quenched and unquenched calculations on comparable lattices in physical units to explore the magnitude of errors due to quenching. Hence, we will compare full QCD calculations using configurations produced by the SESAM collaboration [16] at $\beta=5.6$ with quenched calculations on comparable lattices. Whereas it has been conjectured that the discrepancy between lattice calculations and experimental moments of structure functions arises primarily from quenching, one major result of this work is to show that at the quark masses attainable at present, this explanation is wrong.

The final limitation is errors in calculating the large volume, small quark mass limit. Since the lattice volume must be large enough that the pion Compton wavelength fits well inside and inversion algorithms become less efficient for light quarks, it is presently impossible to perform full QCD calculations with dynamical quark masses corresponding to physical pions or even to pions sufficiently light that chiral perturbation theory gives reliable extrapolations. Thus, a major uncertainty is the extrapolation from the relatively heavy quark 
masses used in lattice calculations to the small quark mass required to produce the physical pion. This is particularly worrisome for calculating hadron structure because of the major role played by the pion cloud in the nucleon. Since a small box and heavy quarks suppress the pion cloud, it is not surprising that the nucleon magnetic moment, much of which comes from the pion current, is low or that the axial charge is too small. Indeed, model estimates 17,18 show that both discrepancies plausibly arise from omission of the full contribution of the pion cloud in present lattice calculations. Hence, a second major result of this work is to show how an extrapolation incorporating the leading effects of chiral symmetry can simultaneously resolve the discrepancy in the lowest three moments of the spin averaged structure function.

The outline of this paper is as follows. Section II provides the background and defines the operators we evaluate. Perturbative renormalization is discussed in Section III and technical details of the lattice calculation are given in Section IV. Section V presents the results, including the overlap of trial wave functions with lattice hadron ground states, comparison of observables calculated with quenched and unquenched configurations to study quenching errors, comparison of observables calculated with cooled and uncooled unquenched configurations to study the role of instantons and their associated zero modes, and comparison of linear and chiral extrapolations with phenomenology. A summary and conclusions are given in Section VI.

\section{BACKGROUND}

\section{A. Moments of nucleon light cone quark distributions}

By the operator product expansion, moments of the linear combinations of quark and antiquark distributions in the proton

$$
\begin{aligned}
\left\langle x^{n}\right\rangle_{q} & =\int_{0}^{1} \mathrm{~d} x x^{n}\left(q(x)+(-1)^{n+1} \bar{q}(x)\right) \\
\left\langle x^{n}\right\rangle_{\Delta q} & =\int_{0}^{1} \mathrm{~d} x x^{n}\left(\Delta q(x)+(-1)^{n} \Delta \bar{q}(x)\right) \\
\left\langle x^{n}\right\rangle_{\delta q} & =\int_{0}^{1} \mathrm{~d} x x^{n}\left(\delta q(x)+(-1)^{n+1} \delta \bar{q}(x)\right),
\end{aligned}
$$

where the quark density, helicity, and transversity [19] distributions

$$
\begin{aligned}
q & =q_{\uparrow}+q_{\downarrow} \\
\Delta q & =q_{\uparrow}-q_{\downarrow} \\
\delta q & =q_{\top}-q_{\perp},
\end{aligned}
$$

are related to the following matrix elements of twist-2 operators

$$
\begin{aligned}
2\left\langle x^{n-1}\right\rangle_{q_{r}} P_{\mu_{1}} \cdots P_{\mu_{n}} & \equiv \frac{1}{2} \sum_{S}\left\langle P S\left|\left(\frac{i}{2}\right)^{n-1} \bar{\psi}^{r} \gamma_{\left\{\mu_{1}\right.} \stackrel{\leftrightarrow}{D}_{\mu_{2}} \cdots \stackrel{\leftrightarrow}{D}_{\left.\mu_{n}\right\}} \psi^{r}\right| P S\right\rangle \\
\frac{2}{n+1}\left\langle x^{n}\right\rangle_{\Delta q_{r}} S_{\{\sigma} P_{\mu_{1}} \cdots P_{\left.\mu_{n}\right\}} & \equiv-\left\langle P S\left|\left(\frac{i}{2}\right)^{n} \bar{\psi}^{r} \gamma_{5} \gamma_{\{\sigma} \stackrel{\leftrightarrow}{D}_{\mu_{1}} \cdots \stackrel{\leftrightarrow}{D}_{\left.\mu_{n}\right\}} \psi^{r}\right| P S\right\rangle \\
\frac{2}{m_{N}}\left\langle x^{n}\right\rangle_{\delta q_{r}} S_{[\mu} P_{\{\nu]} P_{\mu_{1}} \cdots P_{\left.\mu_{n}\right\}} & \equiv\left\langle P S\left|\left(\frac{i}{2}\right)^{n} \bar{\psi}^{r} \gamma_{5} \sigma_{\mu\{\nu} \stackrel{\leftrightarrow}{D}_{\mu_{1}} \cdots \stackrel{\leftrightarrow}{D}_{\left.\mu_{n}\right\}} \psi^{r}\right| P S\right\rangle,
\end{aligned}
$$


Here, $\stackrel{\leftrightarrow}{D} \equiv \vec{D}-\stackrel{\leftarrow}{D}, r$ denotes the quark flavor, $x$ denotes the momentum fraction carried by the quark, $S^{2}=m_{N}^{2},\{\}$ and [] denote symmetrization and antisymmetrization respectively, and the mixed symmetry $[\{]\}$ term is first symmetrized and then antisymmetrized so that it is written explicitly as

$$
O_{\left[\sigma\left\{\mu_{1}\right] \cdots \mu_{n}\right\}}^{5} \equiv \frac{1}{n+1}\left(O_{\sigma \mu_{1} \mu_{2} \ldots \mu_{n}}^{5}-O_{\mu_{1} \sigma \mu 2 \cdots \mu_{n}}^{5}+O_{\sigma \mu_{2} \mu_{1} \cdots \mu_{n}}^{5}-O_{\mu_{1} \mu_{2} \sigma \cdots \mu_{n}}^{5}+\cdots\right) .
$$

We note that the odd moments $\left\langle x^{n}\right\rangle_{q}$ are obtained from the spin-independent structure functions $F_{1}$ or $F_{2}$ measured in deep inelastic electron or muon scattering

$$
\begin{aligned}
& \int_{0}^{1} d x x^{n-1} F_{1}\left(x, Q^{2}\right)=\frac{1}{2} C_{n}^{v}\left(Q^{2} / \mu^{2}\right) \sum_{r} e_{r}^{2}\left\langle x^{n-1}\right\rangle_{q_{r}}(\mu) \\
& \int_{0}^{1} d x x^{n-2} F_{2}\left(x, Q^{2}\right)=C_{n}^{v}\left(Q^{2} / \mu^{2}\right) \sum_{r} e_{r}^{2}\left\langle x^{n-1}\right\rangle_{q_{r}}(\mu),
\end{aligned}
$$

and even moments of $\left\langle x^{n}\right\rangle_{\Delta q}$ are determined from the spin-dependent structure function $g_{1}$

$$
\int_{0}^{1} d x x^{n} g_{1}\left(x, Q^{2}\right)=\frac{1}{4} C_{n}^{a}\left(Q^{2} / \mu^{2}\right) \sum_{r} e_{r}^{2} 2\left\langle x^{n}\right\rangle_{\Delta q_{r}}(\mu),
$$

where $e_{r}$ is the quark's electric charge, and $C_{n}$ denotes the Wilson coefficient. Note that the moments $\left\langle x^{n}\right\rangle_{q}$ and $\left\langle x^{n}\right\rangle_{\Delta q}$ are proportional to the quantities $v_{n+1}$ and $a_{n}$ defined in Ref. [10]

$$
\begin{aligned}
\left\langle x^{n}\right\rangle_{q} & =v_{n+1}^{(q)} \\
\left\langle x^{n}\right\rangle_{\Delta q} & =\frac{1}{2} a_{n}^{(q)} .
\end{aligned}
$$

In addition, the two spin-dependent structure functions $g_{1}$ and $g_{2}$ also determine the quantity $d_{n}$

$$
\frac{1}{n+1} d_{n}^{r} S_{[\sigma} P_{\left\{\mu_{1}\right]} \cdots P_{\left.\mu_{n}\right\}} \equiv-\left\langle P S\left|\left(\frac{i}{2}\right)^{n} \bar{\psi}^{r} \gamma_{5} \gamma_{[\sigma} \stackrel{\leftrightarrow}{D}_{\left\{\mu_{1}\right]} \cdots \stackrel{\leftrightarrow}{D}_{\left.\mu_{n}\right\}} \psi^{r}\right| P S\right\rangle
$$

which is a twist-three operator and does not have a simple interpretation in terms of parton distribution functions [20]. However, since with Wilson fermions, $\left.\gamma_{5} \gamma_{[\sigma} \stackrel{\leftrightarrow}{D}_{\left\{\mu_{1}\right]} \cdots \stackrel{\leftrightarrow}{D} \mu_{n}\right\}$ mixes with the lower dimension operator $\frac{1}{a} \gamma_{5} \gamma_{[\sigma} \gamma_{\left\{\mu_{1}\right]} \cdots \overleftrightarrow{D}_{\left.\mu_{n}\right\}}$, it is not possible to compare with phenomenological results using the perturbative renormalization constants and mixing coefficients calculated in this work. Either nonperturbative renormalization is required [21] as has been carried out in [55], or the operators need to be recalculated with overlap fermions [23] or some alternative formulation for which mixing with lower dimension operators does not occur .

Even moments $\left\langle x^{n}\right\rangle_{q}$ are obtained from deep inelastic neutrino scattering, and in addition, a variety of other processes have contributed to what is now a detailed empirical knowledge of the quark and antiquark distributions in the nucleon. Hence, we will subsequently compare our results with moments calculated from the CTEQ, GRV, MRS, GRSV, and GS and global fits to the world supply of data [1 5]. The moments of parton distributions $\left\langle x^{n}\right\rangle_{q}(\mu)$, $\left\langle x^{n}\right\rangle_{\Delta q}(\mu)$, and $\left\langle x^{n}\right\rangle_{\delta q}(\mu)$ are scheme and scale dependent, and we will convert our lattice matrix elements to the $\overline{M S}$ scheme and evaluate them at the scale $\mu^{2}=\frac{1}{a^{2}} \sim 4 \mathrm{GeV}^{2}$. 


\section{B. Lattice operators}

Our objective is to calculate matrix elements of traceless, appropriately symmetrized and antisymmetrized operators of the general form

$$
\mathcal{O} \equiv \bar{\psi} \Gamma \gamma_{\mu_{1}} \stackrel{\leftrightarrow}{D}_{\mu_{2}} \cdots \stackrel{\leftrightarrow}{D}_{\mu_{n}} \psi
$$

where $\Gamma=1, \gamma_{5}$, or $\gamma_{5} \gamma_{\sigma}$, on a hypercubic lattice to approximate the corresponding continuum operators as accurately as possible. Hence we choose representations of the hypercubic group, $\mathrm{H}(4)$ [24], to eliminate operator mixing as much as possible, and after fulfilling this objective, to minimize statistical errors by including as few nonzero components of the nucleon momentum as possible.

Since H(4) is a subgroup of the Lorentz group, irreducible representations of the Lorentz group are in general reducible under the $\mathrm{H}(4)$ group, and we choose the representation to optimize the approximation. It is essential to choose a representation that does not mix with lower dimension operators, since the coefficients would increase as $1 / a^{n}$ in the continuum limit. In addition, because of the possible inaccuracy of perturbative mixing coefficients and the difficulty of determining mixing coefficients nonperturbatively, it is desirable to avoid mixing with operators of the same dimension as well. In choosing between operators with the same mixing properties, it is desirable to use a nucleon source with as few nonzero spatial momentum components as possible, since each projection introduces substantial stochastic noise. In fact, we will subsequently show that with available configurations, it is not possible to obtain adequate statistics in any momentum sector other than $\vec{p}=0$. Since any expectation value of an operator with tensor index $j$ is proportional to $P_{j}$ (or $S_{j}$ for spin dependent quark distributions), the nucleon must have an additional momentum component projection for each new distinct tensor index that is added to an operator. Hence, the goal is to limit the number of distinct spatial indices. Eventually, as one proceeds to higher moments of quark distributions, all the space-time indices are exhausted and it becomes impossible to avoid mixing with lower dimension operators.

The representations we have chosen for our operators using these criteria are enumerated in Table II. To illustrate the selection process, we describe selection of the spin-independent operators and analogous analysis yields the remaining operators.

To measure $\langle x\rangle_{q}$ one needs to calculate matrix elements of the traceless part of the operator $\bar{q} \gamma_{\{\mu} \stackrel{\leftrightarrow}{D}_{\nu\}} q$, which belongs to the representation $(1,1)$ in the continuum decomposition

$$
\left(\frac{1}{2}, \frac{1}{2}\right) \otimes\left(\frac{1}{2}, \frac{1}{2}\right)=(0,0) \oplus(1,0) \oplus(0,1) \oplus(1,1) .
$$

On the lattice, the nine dimensional representation $(1,1)$ splits into two irreducible representations, $\mathbf{3}_{1}^{+}$and $\mathbf{6}_{3}^{+}$, both of which are symmetric and traceless, where the notation for representations is described in the caption of Table [1]. As a consistency check, it is desirable to calculate operators from each representation. For the first operator, denoted $\langle x\rangle_{q}^{(a)}$, we select the basis vector of $\mathbf{6}_{3}^{+}$:

$$
\left\langle P\left|\bar{q} \gamma_{\{1} \stackrel{\leftrightarrow}{D}_{4\}} q\right| P\right\rangle=2\langle x\rangle_{q}^{(a)} \cdot P_{\{1} P_{4\}},
$$


and for $\langle x\rangle_{q}^{(b)}$, we choose the basis vector of $\mathbf{3}_{1}^{+}$:

$$
\left\langle P\left|\bar{q} \gamma_{4} \stackrel{\leftrightarrow}{D}_{4} q-\frac{1}{3}\left(\bar{q} \gamma_{1} \stackrel{\leftrightarrow}{D}_{1} q+\bar{q} \gamma_{2} \stackrel{\leftrightarrow}{D}_{2} q+\bar{q} \gamma_{3} \stackrel{\leftrightarrow}{D}_{3} q\right)\right| P\right\rangle=2\langle x\rangle_{q}^{(b)} \cdot\left(P_{4} P_{4}-\frac{1}{3} \vec{P}^{2}\right)
$$

Note that since $\langle x\rangle_{q}^{(b)}$ involves $\gamma_{4} \stackrel{\leftrightarrow}{D}_{4}$, it can be measured with $\vec{P}=0$ whereas since $\langle x\rangle_{q}^{(a)}$ involves $\gamma_{1} \stackrel{\leftrightarrow}{D}_{4}$, it requires a state projected onto non-zero $P_{1}$

For $\left\langle x^{2}\right\rangle_{q}$, none of the three (symmetric) representations $4_{1}^{-}$is appropriate, since they are not traceless and hence mix with lower-dimensional operators. The only representations with two distinct indices are the one $\mathbf{8}_{1}^{+}$, which is not symmetric and must therefore be rejected, and the two $\mathbf{8}_{1}^{-}$'s

$$
\bar{q}\left(\gamma_{4} \overleftrightarrow{D}_{1} \stackrel{\leftrightarrow}{D}_{1}-\frac{1}{2}\left(\gamma_{4} \overleftrightarrow{D}_{2} \stackrel{\leftrightarrow}{D}_{2}+\gamma_{4} \overleftrightarrow{D}_{3} \overleftrightarrow{D}_{3}\right)\right) q
$$

and

$$
\bar{q}\left(\gamma_{1} \overleftrightarrow{D}_{4} \stackrel{\leftrightarrow}{D}_{1}+\gamma_{1} \overleftrightarrow{D}_{1} \stackrel{\leftrightarrow}{D}_{4}-\frac{1}{2}\left(\gamma_{2} \overleftrightarrow{D}_{4} \overleftrightarrow{D}_{2}+\gamma_{2} \overleftrightarrow{D}_{2} \overleftrightarrow{D}_{4}+\gamma_{3} \overleftrightarrow{D}_{4} \overleftrightarrow{D}_{3}+\gamma_{3} \overleftrightarrow{D}_{3} \overleftrightarrow{D}_{4}\right)\right) q
$$

which mix as discussed in Refs. 25 27.

For $\left\langle x^{3}\right\rangle_{q}$ the following representations have positive charge conjugation and do not mix with lower dimensional operators: $\mathbf{2}_{1}^{+}, \mathbf{2}_{2}^{+}, \mathbf{3}_{2}^{+}, \mathbf{3}_{2}^{+}, \mathbf{3}_{3}^{+}, \mathbf{6}_{2}^{+}$and $\mathbf{6}_{4}^{+}$. However, the only representations that require a single non-zero momentum component are the two $\mathbf{2}_{1}^{+}$'s, which generically could mix with each other but do not mix at the one loop level for Wilson or overlap fermions [26,23,28].

Note that in addition to the mixing discussed above, in full QCD there is also mixing between gluonic operators and flavor-singlet fermion operators for moments of the quark density and helicity that will not be considered in this work because we have not yet evaluated lattice matrix elements of the relevant gluon operators.

\section{PERTURBATIVE RENORMALIZATION}

Since the phenomenological light cone quark distributions with which we compare our lattice results are extracted from experimental data using the $\overline{M S}$ renormalization scheme, we have converted our lattice calculations to the $\overline{M S}$ scheme in 1-loop perturbation theory using

$$
O_{i}^{\overline{M S}}\left(Q^{2}\right)=\sum_{j}\left(\delta_{i j}+\frac{g_{0}^{2}}{16 \pi^{2}} \frac{N_{c}^{2}-1}{2 N_{c}}\left(\gamma_{i j}^{\overline{M S}} \log \left(Q^{2} a^{2}\right)-\left(B_{i j}^{L A T T}-B_{i j}^{\overline{M S}}\right)\right)\right) \cdot O_{j}^{L A T T}\left(a^{2}\right) .
$$

The anomalous dimensions $\gamma_{i j}$ and the finite constants $B_{i j}$ are given in Table III for Wilson fermions and the specific operators considered.

The $Z$ factors that convert lattice results to the $\overline{M S}$ scheme at scale $Q^{2}=1 / a^{2}$ are equal to

$$
Z\left(g_{0}^{2}=6 / \beta\right)=1-\frac{g_{0}^{2}}{16 \pi^{2}} \frac{4}{3}\left(B^{L A T T}-B^{\overline{M S}}\right)
$$


and are tabulated for two typical values of $\beta$. Note that all of the moments of quark distributions calculated in this work will be presented at the scale of $\mu^{2}=4 \mathrm{GeV}^{2}$ in the $\overline{M S}$ scheme.

Details of perturbative renormalization may by found in Refs. 29, 30, 26]. The results in Table [II] are taken from Refs. [30,23], in which the renormalization factors of $\langle x\rangle_{\delta q}$ and $d_{1}$ for Wilson fermions were calculated for the first time and the remaining operators were checked with earlier results in Refs. [28, 31, 25, 10, 26, 32, revealing a discrepancy in the case of $\left\langle x^{3}\right\rangle_{q}$.

\section{LATTICE CALCULATIONS}

In this section, we describe salient aspects of our lattice calculation of proton matrix elements of the operators corresponding to moments of parton distributions. Details may be found in Ref. [33].

\section{A. Connected Diagrams}

Proton matrix elements of the operators in Eq. 3 are calculated by evaluating the connected and disconnected diagrams shown in Fig. 1. Note that the term "disconnected" refers to diagrams in which the quarks are disconnected but of course as shown in Fig. 1, the overall Feynman diagram is still connected by gluons. It is important to recognize at the outset that although the connected diagrams involve three propagators naively corresponding to the "valence" quarks in a simple hadron model, the contributions of the connected diagrams do not necessarily correspond to the valence parton distribution $q_{v a l} \equiv q-\bar{q}$ defined in phenomenological analyses of high energy scattering data. Rather, as shown in Eq. 1, the odd moments of $\left\langle x^{n}\right\rangle_{q}$ and $\left\langle x^{n}\right\rangle_{\delta q}$ and even moments of $\left\langle x^{n}\right\rangle_{\Delta q}$ measured in deep inelastic lepton scattering correspond to the sum $q+\bar{q}$ and only the even moments of $\left\langle x^{n}\right\rangle_{q}$ and $\left\langle x^{n}\right\rangle_{\delta q}$ and odd moments of $\left\langle x^{n}\right\rangle_{\Delta q}$ correspond to the difference $q-\bar{q}$.

It is technically much more difficult to evaluate the disconnected diagrams than connected diagrams and results using the eigenmode expansion technique that has recently been developed for this purpose [34] are not yet available. Therefore, in the present work, we will compare the contributions of connected diagrams with flavor non-singlet combinations of experimental results. Because the coupling of the disconnected loop to the rest of the diagram is flavor independent, the disconnected diagrams do not contribute to the difference between the moments for degenerate up and down quarks. For example, the axial charge can be calculated directly from differences of connected contributions, $g_{A}=\langle\Delta u+\Delta \bar{u}-\Delta d-\Delta \bar{d}\rangle_{\text {connected }}$. Hence, we will subsequently compare our results with moments of flavor non-singlet combinations of the sum or differences of phenomenological quark and antiquark distributions.

One technical difference between our calculations and those of other works is the fact that we use Dirichlet boundary conditions for the valence fermions in the time direction. With periodic or antiperiodic boundary conditions, contributions from the images of the sources and sinks restrict the useful source-sink separation to less than half the total time extent. Dirichlet boundary conditions prevent propagation from these image sources and 
allow utilization of a larger fraction of the lattice volume for ground state operator measurements. We note that the closely related Schrödinger functional boundary conditions used in a calculation of the average quark momentum in the pion provide similar benefits [35].

\section{B. Sources}

To facilitate measurement of proton matrix elements, we have optimized the overlap between a computationally efficient nucleon source and the proton ground state. The starting point is the interpolating field for the proton

$$
J=\epsilon^{a b c}\left[u^{a} C \gamma_{5} d^{b}\right] u^{c}
$$

where $C$ denotes the charge conjugation matrix. This field corresponds to the non-relativistic quark model wave function $\epsilon^{a b c}\left[u_{\uparrow}^{a} d_{\downarrow}^{b}-u_{\downarrow}^{a} d_{\uparrow}^{b}\right] u_{\uparrow}^{c}$ in the non-relativistic limit. Since truncation of the lower components does not significantly reduce its overlap with the physical proton [36] we save an overall factor of two in computation and storage by only calculating propagators from the upper components of the source.

As shown below in Section $\nabla$, if $J$ in Eq. 12 is a point source, the overlap with the nucleon ground state is of the order $10^{-4}$, so generalization to finite spatial extent is desirable. Since the source must be factorizable in order for matrix elements to be calculated from single quark propagators, we use distributed quark fields with adjustable spatial extent. In ref [37, it was shown that gauge fixed sources with gaussian wave functions and gauge invariant smeared Wuppertal sources [38,39] produced comparable results, and for computational convenience we use the latter.

The smeared wave function is defined [40]

$$
\psi^{(N, \alpha)}=(1+\alpha H)^{N} \psi^{0} \quad \text { where } \quad H=\sum_{i=1}^{3}\left(U_{n, i}+U_{n-\hat{i}, i}^{\dagger}\right) \quad \text { and } \quad \psi^{0}(n)=\delta_{n n_{0}}
$$

with the spatial extent being controlled by the coefficient of the nearest neighbor hopping term, $\alpha$, and the number of smearing steps, $N$. The distribution is approximately Gaussian with an equilibrated gauge field $U$ producing a narrower distribution than for the free case. A convenient measure of the smearing is the rms radius

$$
r_{r m s}=\left\langle r^{2}\right\rangle^{\frac{1}{2}}=\left[\frac{\int d^{3} x r^{2} \psi^{*} \psi}{\int d^{3} x \psi^{*} \psi}\right]^{\frac{1}{2}}
$$

and figure 2 shows how $r_{r m s}$ depends on the parameters $N$ and $\alpha$. As one expects from the free case and from the fact that smearing is a random walk governed by the gauge fields, the rms radius is approximately proportional to $\sqrt{N}$. Note that the size of the source is nearly independent of $\alpha$ for $\alpha>3$, at which point the constant term in Eq. 13 becomes negligible relative to the hopping term. In the calculations described in section $\nabla$, we set $\alpha=3$ and use $N$ to adjust $r_{r m s}$ to optimize the source.

The source is optimized by maximizing the overlap between the normalized state created by the action of the source on the QCD vacuum $\left|\Psi_{J}\right\rangle=\mathcal{N} \bar{J}|\Omega\rangle$ and the normalized ground state of the proton $|0\rangle$. Denoting the momentum projected normalized eigenstates of the 
proton by $|n\rangle$ and their energies by $E_{n}$, the momentum projected two point correlation function may be expanded:

$$
\langle J(t) \bar{J}(0)\rangle=\mathcal{C} \sum_{n}\left|\left\langle\psi_{J} \mid n\right\rangle\right|^{2} e^{-E_{n} t}
$$

where $\mathcal{C}$ is an unknown normalization constant. Since one can directly measure the correlation function at zero time separation

$$
A=\langle J(0) \bar{J}(0)\rangle=\mathcal{C} \sum_{n}|\langle J \mid n\rangle|^{2}
$$

and reliably fit the large $t$ behavior of the correlation function to extract the ground state contribution

$$
B=\mathcal{C}|\langle J \mid 0\rangle|^{2},
$$

the probability that the source contains the proton ground state is given by

$$
P(0)=\frac{B}{A}=\left|\left\langle\Psi_{J} \mid 0\right\rangle\right|^{2} \text {. }
$$

The importance of maximizing the overlap of the source with the proton is particularly clear when one considers the three point function used to calculate matrix elements:

$$
\left\langle J\left(t_{3}\right) \mathcal{O}\left(t_{2}\right) J\left(t_{1}\right)\right\rangle=\mathcal{C} \sum_{n, m}\left\langle\psi_{J} \mid n\right\rangle\langle n|\mathcal{O}| m\rangle\left\langle m \mid \psi_{J}\right\rangle e^{-E_{n}\left(t_{3}-t_{2}\right)-E_{m}\left(t_{2}-t_{1}\right)} .
$$

Although the contribution of a contaminant $\left\langle\psi_{J} \mid n\right\rangle \sim \epsilon$, to the two point function, Eq. 14, is of order $\epsilon^{2}$, the contribution to the three point function, Eq. 18 is of order

$\epsilon \frac{\langle n|\mathcal{O}| 0\rangle}{\langle 0|\mathcal{O}| 0\rangle}$. Depending on the off-diagonal elements of any particular operator, the error when the operator is close to one of the sources may be substantial, and the sign of the contribution is undetermined. Specific examples will be seen in plateau plots presented in Section $\square$.

\section{Sequential Propagators}

There are two alternative strategies for calculating the connected diagrams in Fig. 1 with sequential propagators. Using a propagator from a fixed source to all points $x_{0}$ times the operator $\mathcal{O}\left(x_{0}\right)$ as a new source to propagate to an arbitrary sink location allows one to calculate matrix elements of the operator between all source sink separations [13, 114, 41, 44]. The matrix element is then obtained from the linear term in the time separation between the source and sink. The SESAM collaboration has used this variable time extent method to calculate the axial charge [14] for the configurations used in this work, and we will compare our results with theirs in Section $\square$. However, the fact that a new set of propagators must be calculated for each operator makes this alternative too costly for the large set of operators of interest in this present work.

The alternative we will use is to combine two forward propagators from a fixed source, smear them and momentum project on a specific time slice to create a sink, and use this sink as a new source for a backward going propagator [45]. The matrix element for any operator can then be obtained by combining forward and backward propagators with that operator. Because the time separation between source and sink is fixed in this approach, the effect of this separation has been studied and optimized as described in Section $\square$. The details of calculating matrix elements with these sequential propagators are described in Appendix A. 


\section{Lattice Data Analysis}

To ensure that the systematic and statistical errors are well understood and controlled, we have used several methods to analyze the lattice measurements of matrix elements.

In our primary analysis, which is used for all the results presented in tables and graphs, we define a central window for the location of the insertion of the measured operator $\left[t_{\text {source }}+\right.$ $\left.\delta t, t_{\text {sink }}-\delta t\right]$ in which contamination from excited states in the source or sink has negligible effect, average the ratio of three-point to two-point functions over this window, and use the jackknife method [46] to estimate the average value and variance. For smeared sources, we used $\delta t=3$ and for tests with unsmeared sources we have also used $\delta t=5$.

As a secondary analysis, to ensure that we have avoided systematic errors from excited state contaminants in the plateau region, we have also fitted the phenomenological form

$$
R(t)=R_{0}+\sum_{i}\left(b_{i} e^{-c_{i} t}+b_{i} e^{-c_{i}(T-t)}\right)
$$

over the whole range between the source and the sink. The exponentials correcting for the contaminants are symmetric because we have used the same smearing in the source and sink, and the value for the matrix element is $R_{0}$.

From our data, it was only possible to determine one pair of exponentials, and we used a jackknife estimate on the fitted values of $R_{0}$ to determine the error. For operators for which the errors were small enough that the exponential contaminants were well determined and stable, the plateau and exponential fits produced statistically consistent results with comparable errors. This ensures that our primary results do not have statistically significant bias from excited states. In some cases, the exponential contaminants were poorly determined and produced spurious fluctuations in the analysis which overestimated the actual statistical fluctuations in the plateau region. This is the reason we selected the plateau fits as our primary analysis and view the exponential fits as a secondary consistency check.

To further ensure that we understood our statistical errors, we also examined bootstrap distributions 47 for all our observables at the lightest SESAM quark mass. An ensemble of size $M$ was created by randomly picking configurations from the original sample with replication, and typical distributions of values of measurements of $\langle x\rangle_{q}^{(b)}$ are shown in Fig. (3), where $M=2000$ and the sample sizes in the left and right figures are 25 and 204 configurations respectively. Three error bars are shown in each case: the basic jackknife error bar which is calculated independently of the bootstrap procedure, the $68 \%$ confidence interval based on the bootstrap distribution, and the statistical variance of the bootstrap measurements. The three arrows correspond to the jackknife result, the median of the distribution, and the mean of the distribution. This figure shows that for a sample size as small as 25, the distribution is far from Gaussian, the three error bars differ significantly, and even the arrows disagree slightly. For the large sample size 204 used in our full data analysis, everything is consistent: the distribution is nearly Gaussian, the three errors are consistent, and the three arrows are equivalent. Thus, the bootstrap analysis gives added assurance that the statistics are understood and under control. 


\section{LATTICE RESULTS}

This section presents the results of our calculations of moments of quark distribution functions in full and quenched QCD, as well as relevant tests of the lattice technology and consistency checks.

\section{A. Lattice Gluon Configurations}

The parameters specifying the full QCD, quenched, and cooled configurations used in our calculations are tabulated in Table I. The full QCD configurations were calculated using the hybrid Monte Carlo algorithm by the SESAM collaboration [16 at $\beta=5.6$ and by the SCRI collaboration [48] at $\beta=5.5$. The quenched configurations were calculated at MIT at $\beta=6.0$ to be directly comparable in lattice spacing to the unquenched $\beta=5.6$ configurations, and the cooled configurations were obtained by applying 50 cooling sweeps to the SESAM configurations as described later.

The practical limitations associated with these configurations pose significant problems in extrapolating to both the chiral and continuum limits as required to compare with phenomenological data.

\section{Chiral extrapolation}

A common criterion to keep the pion correlation length significantly smaller than the physical size of the lattice and to avoid unphysical interactions with periodic images is to require that the pion Compton wavelength be less than one-fourth the spatial dimension of the lattice. Because resources limit us to $16^{3} \times 32$ lattices, at $\beta=5.6$ where the lattice spacing $a=0.091 \mathrm{fm}$, the lattice dimension is only $1.46 \mathrm{fm}$ so that the pion mass must be greater than $540 \mathrm{MeV}$. However even for the lowest SESAM quark mass, which exceeds this criterion, there are significant discrepancies between mass measurements on $16^{3} \times 32$ and $24^{3} \times 48$ lattices [49], indicating serious finite volume effects. Hence, in our final analysis, we have only included the heaviest three SESAM quark masses in our results.

As discussed in detail in the final section, much of the physics of the pion cloud of the nucleon is omitted on a lattice of size $1.46 \mathrm{fm}$ with pions heavier than $540 \mathrm{MeV}$, so it is unreasonable to expect naive linear extrapolation of calculations in this regime to accurately include the quantitatively important physics of the pion cloud. However, since we do not presently have data in a regime in which we can fully determine the parameters of an extrapolation incorporating the behavior known from chiral perturbation theory, most of our data analysis will be based on naive linear extrapolation. This extrapolation is still useful because it crisply frames the issues that need to be confronted in comparison of $a b$ initio lattice calculations with experiment.

\section{Continuum extrapolation}

Due to limitations in presently available configurations for Wilson fermions, extrapolation to the continuum limit is even more problematic. Table IV summarizes parameters and lattice spacings for configurations that are presently available for dynamical Wilson fermions. Since we are calculating nucleon properties, we believe it is most consistent to set the scale using the lattice spacing determined from the nucleon mass, $a_{N}$. Unfortunately, there is a large discrepancy in determinations of $a_{N}$, and for qualitative purposes we have assumed 
the behavior given by the LANL results, since this is the only data set in which one group calculated consistently at three different $\beta$.

One goal has been to use a comparison of calculations of moments of quark distributions using SCRI configurations at $\beta=5.5$ and SESAM configurations at $\beta=5.6$ to obtain an indication of the finite lattice size dependence, and we report results from both $\beta$ 's below. However, the problem of finite size effects is shown in Fig. 4, where we present scaled values of $m_{\pi}^{2}$ at different values of $\beta$ for the data sets in Table $\mathbb{I V}$. The scaled mass squared, $\left(a m_{\pi}\right)^{2} \times\left(a_{5.6} / a\right)^{2}$, converts the mass in different lattice units at each $a$ to fixed lattice units at $\beta=5.6$ for ease of comparison. Thus, the ordinate is proportional to $m_{\pi}^{2}$ in physical units, and 0.05 corresponds to $m_{\pi}^{2}=0.24 \mathrm{GeV}^{2}$ or $m_{\pi}=490 \mathrm{MeV}$. The dotted line shows the point at which the pion Compton wavelength equals one fourth of the spatial dimension. Given that the lowest SESAM point has significant finite size errors, we expect the lowest two SCRI points to have comparable contamination from finite size effects. Since there are no SCRI points comparable to the highest SESAM points, there is no way to delete the lowest SCRI points and perform an extrapolation comparable to the SESAM extrapolation. Hence, we believe that the differences between moments of quark distributions calculated with the SCRI and SESAM configurations is an undetermined combination of lattice spacing errors and finite volume effects. New dynamical Wilson quark calculations are underway at $\beta=5.5$ and $\beta=5.3$ to separate these finite volume and finite lattice spacing effects.

\section{B. Source optimization}

To obtain as much physics as possible from the SESAM and SCRI configurations, the nucleon sources have been optimized in their overlap with the ground state and with respect to their separation in Euclidean time.

As discussed in section IVB, the rms radius, $\left\langle r^{2}\right\rangle^{\frac{1}{2}}$, characterizing the spatial extent of a Wuppertal source was varied to maximize the overlap of the source with the ground state proton wave function, $P(0)=\left|\left\langle\Psi_{J} \mid 0\right\rangle\right|^{2}$. Figure 5 shows the overlap, with jackknife errors, between the smeared source and the proton ground state as a function of the source rms radius in lattice units, calculated at $\beta=5.6, \kappa=0.1575$, and smearing parameter $\alpha=3$. The number of smearing steps, $N$, to produce the rms radii in this plot ranges from 20 at the lowest non-zero point to 250 for the highest. It is interesting that the overlap increases from $6 \times 10^{-5}$ for a point source to 0.5 for an rms radius of the order of 4.5 lattice units. Clearly, this four-order-of-magnitude increase is a dramatic aid in improving the plateau for measurements. Based on these and analogous calculations at $\beta=5.5$, the optimal Wuppertal smearing occurs at a physical rms radius of $0.4 \mathrm{fm}$, which corresponds to $\alpha=3$ and $N=50$ at $\beta=5.6$ and $\alpha=3$ and $N=30$ at $\beta=5.5$.

Note that this overlap calculation opens the possibility of performing instructive variational studies of the nucleon ground state. Already, a very simple quark and gluon wave function made up a product of three gaussian single-particle wave functions smeared with surrounding links of glue has a $50 \%$ overlap with the full ground state and is measured with good statistical accuracy. Hence, it should be straightforward and practical to investigate variationally the extent to which the wave function can be improved by the addition of changes in the quark wave function, such as the addition of diquark correlations, or of changes in the gluon wave function. 
The optimal separation between source and sink is a compromise between two competing effects. As the separation increases, the size of the usable plateau increases, reducing systematic error and in principle allowing for increased statistical accuracy from averaging over an increasingly large region. However, the propagators themselves are exponentially decreasing with separation and the fractional error with which they can be measured increases exponentially. (This is analogous to the exponential error growth in the measurement of Wilson loops or Polyakov lines, but because the measurement of the three-point function is intrinsically non-local, it is not amenable to the error reduction techniques of Lüscher and Weisz [50]).

To determine the optimal compromise between these two competing effects more quantitatively, plateaus were calculated as a function of the source-sink separation for point sources, and the results for $\langle x\rangle_{q}^{(b)}$ using 100 configurations at $\beta=5.6$ and $\kappa=.1575$ are shown in Fig. 6 for time separations $\Delta T=12$ and 14. The errors for $\Delta T=14$ are $50 \%$ larger than for $\Delta T=12$, and become prohibitive for larger separations. This is consistent with the results of ref [10, that for a $16^{3} \times 32$ lattice in quenched QCD with $\beta=6.0$, which has a comparable lattice spacing, the error bars grow significantly for $\Delta T>13$. Although the plateau region for $\Delta T=14$ is hardly present at $\Delta T=12$ for point sources, one may still extract the correct matrix element using the exponential fit method described in section IVD. Furthermore, using optimal smeared sources to enhance the ground state overlap by four orders of magnitude decreases the excited state contaminants to the point that a well defined central plateau is recognized for $\Delta T=12$ as shown below. Hence, to reduce the overall statistical errors in our calculations, it is optimal to use a physical separation of approximately $1.1 \mathrm{fm}$ which corresponds to $\Delta T=12$ for SESAM configurations at $\beta=5.6$ and to $\Delta T=10$ for SCRI configurations at $\beta=5.5$.

The final quality of the plateaus used for measurements of operators in a zero momentum ground state are shown in Fig. 7, where we plot measurements of the operators $\langle x\rangle_{q}^{(b)},\langle 1\rangle_{\Delta q},\langle x\rangle_{\Delta q}^{(b)}$, and $\langle 1\rangle_{\delta q}$ as a function of Euclidean time for an ensemble of 200 SESAM configurations with $\beta=5.6$ and $\kappa=0.1560$. The improvement in the plateau for $\langle x\rangle_{q}^{(b)}$ produced by smearing is seen by comparing the right panel of Fig. 6 and the upper left panel of Fig. 17. Note also, as emphasized in connection with Eq. 18, that the sign of the exponential contaminant near the source or sink may be either positive or negative, with a small negative contribution arising in the case of the axial charge, $\langle 1\rangle_{\Delta q}$ shown in the upper right panel of Fig. 17. These well-defined plateaus are typical of all our measurements of operators in a zero momentum ground state and show why we obtain consistent results with plateau and exponential fits and satisfactory statistics. In the case of projection onto non-zero momentum ground states, errors are substantially larger. Although we also report these non-zero momentum results in this work, the errors are generally unsatisfactory and additional calculations will be carried out to improve their statistics.

One other optimization was investigated, but turned out to produce minimal improvement. There is considerable freedom in selecting lattice operators from the irreducible representations tabulated in Table [1]. For example, instead of the basis vector $\bar{q} \gamma_{4} \stackrel{\leftrightarrow}{D}_{4} q-\frac{1}{3}\left(\bar{q} \gamma_{1} \stackrel{\leftrightarrow}{D}_{1} q+\bar{q} \gamma_{2} \stackrel{\leftrightarrow}{D}_{2} q+\bar{q} \gamma_{3} \stackrel{\leftrightarrow}{D}_{3} q\right)$ of representation $3_{1}^{+}$, one could equally well chose $\bar{q} \gamma_{4} \stackrel{\leftrightarrow}{D}_{4} q-\bar{q} \gamma_{i} \stackrel{\leftrightarrow}{D}_{i} q$ for $i=1,2$, or 3 . Since the direction $i=3$ is inequivalent to the other two spatial directions because the spin is polarized in the 3 direction, one can find numerically the optimal combination of the basis vectors that minimizes the variance in the measure- 
ment of $\langle x\rangle_{q}^{(b)}$. However, in the end, the reduction in the statistical error is only about $7 \%$, so this refinement was not implemented in our production calculations.

\section{Consistency checks}

This section summarizes a number of consistency checks that have been performed to ensure the reliability and accuracy of our calculations of moments of quark distributions.

\section{Sources}

One potential pitfall of optimizing sources to increase the size of the plateau region is the possibility that although excited state contaminants appear to have been diminished, unobserved systematic errors may have sneaked into the plateau measurements. To ensure that such systematic errors have not been introduced into our present calculations, we have evaluated the operators of interest for various combinations of source and sink combinations. Figure 8 compares the results for $\langle x\rangle_{q}^{(b)},\langle 1\rangle_{\Delta q}$, and $\langle 1\rangle_{\delta q}$ calculated using point-point, pointsmeared, smeared-point and smeared-smeared source sink combinations, showing that in all cases the results are statistically consistent. Forty configurations at $\beta=5.6$ were used with a source-sink separation of 12 , and the smeared sources had $N=20$ smearing steps. Each cluster of four error bars corresponds to four different window sizes over which the measurements were averaged, characterized by the number of points omitted at the source and sink. The case shown in Fig. 7, denoted (3,3), corresponds to omission of 3 points at the source and 3 points at the sink and is shown at the left of each cluster. With a separation of 12 lattice spacings, there are 13 points in all, of which 6 are deleted leaving a measurement plateau of 7 . The remaining error bars in each cluster correspond to $(3,5)$, $(5,3)$, and $(5,5)$ points omitted at the source and sink respectively. Thin lines denote cases in which the window comes too close to either a point sink or point source and overlaps with a region with contamination from excited states and the error bars denote jackknife errors.

Figure 9 shows a related comparison of the measurements of the same three observables in which the source and sink are smeared with $N=0, N=20$, and $N=100$ smearing steps. The window sizes within each cluster of error bars are the same as in the previous case. Again, the observables are consistent for all three cases, although the error bars become substantially larger for the largest smearing because the size of the paths of link variables generated by the smearing increases significantly.

\section{Boundary conditions}

The Dirichlet boundary conditions in Euclidean time we have used enable us to calculate the exponential decay of two point functions far beyond the midpoint of the lattice without the usual contributions of the propagation of the parity partner of the nucleon from first images in the time direction. Only within a few lattice points of the edge of the lattice do artifacts associated with reflection from the boundary (or equivalently, negative image charges) become significant. To verify that the choice of boundary condition does not affect the measured values of hadronic matrix elements, Fig. 10 shows the results of measuring the operators $\langle x\rangle_{q}^{(b)},\langle 1\rangle_{\Delta q},\langle 1\rangle_{\delta q}$, and $\langle x\rangle_{\Delta q}$ using Dirichlet and periodic boundary conditions. Note that although the results are statistically consistent, our choice of Dirichlet boundary conditions significantly reduces the error bars for some operators, such as $\langle x\rangle_{q}^{(b)}$ and $\langle 1\rangle_{\Delta q},\langle 1\rangle_{\delta q}$.

\section{Precision}


In our production calculations, the conjugate gradient inversion to calculate quark propagators was performed in single precision (to increase performance on the ES-40 cluster) using a perturbative stopping residue $r_{\min }^{2}=10^{-14}$ and four restarts [51]. To verify that single precision with the restart procedure provided adequate precision, we show the discrepancy between calculations of the axial charge, $\langle 1\rangle_{\Delta q}$, calculated in single and double precision as a function of the residual $r_{\min }^{2}$ in Fig. 11. We note that all discrepancies are already negligible at residue $r_{\min }^{2}=10^{-12}$, providing a two order of magnitude safety margin.

\section{Autocorrelation functions}

Autocorrelation functions were calculated for lattice measurements of $\langle x\rangle_{q}^{(b)}$ and $\langle x\rangle_{\Delta q}$ using the SESAM ensemble at $\kappa=0.1575$ consisting of 200 configurations separated by 25 Hybrid Monte-Carlo trajectories. Correlations for all non-zero configuration separations were statistically consistent with zero, and all indications suggest that the 200 configurations are indeed independent for all the degrees of freedom relevant for the present measurements.

In contrast to the SESAM configurations, the SCRI configurations are only separated by 10 trajectories. To minimize the possible effect of correlations, the physical location of the source and sink was shifted by 16 time slices on all odd configurations relative to those on even configurations. Autocorrelation functions for 200 SCRI configurations at $\kappa=0.1600$ yielded a result statistically inconsistent with zero only for separation by two configurations, in which case the correlation was approximately $0.2 \pm 0.15$. Thus for the degrees of freedom relevant to our proton matrix elements, configurations separated by 10 Hybrid Monte-Carlo trajectories with sources and sinks displaced by 16 time slices are independent, whereas those separated by 20 Hybrid Monte-Carlo trajectories with the sources and sinks at the same location still have non-vanishing correlations. The relative independence of measurements at different source and sink positions provides an attractive means of extracting additional physics from the available full QCD configurations that will be exploited in subsequent calculations.

\section{Two and three point functions}

The nucleon two point function may be calculated in two ways: by combining at the sink three forward propagators calculated from the source, or by combining a backward propagator with the sequential source. The agreement within roundoff error of these two results was used not only as a check on source methodology, but also at run time as a test to verify that stored forward and backward propagators had not been corrupted.

Another check is to verify that the spatial integral of the matrix element of the conserved nonlocal vector current (the Noether current for the discrete Wilson lattice action), $\left\langle P\left|\int d^{3} x J_{0}^{(u), N L}\right| P\right\rangle /\langle P \mid P\rangle$, equals 2 or 1 for up and down quarks respectively. This relation must be satisfied configuration by configuration and is also used as a run-time test of the configurations.

An additional test of the source and sink construction is to project the sink onto a fixed position in the sink time-slice, rather than momentum projecting it, and to replace all link variables by their mirror images. We have verified that the resulting three-point function is the mirror image of the original result configuration by configuration.

Finally, as discussed previously, matrix elements of operators may either be calculated with the variable time extent method, in which one sums over all times at which the operator insertion could occur and determines the slope of the dependence on $t_{\text {sink }}$, or by measuring the plateau using the fixed source-sink separation as done in this work. A consistency check 
that is satisfied configuration by configuration is that the sum of the three-point function over the intermediate time at which the operator is measured for a fixed source-sink separation should be equal to the variable time extent three point function for the fixed source-sink separation. In addition, we have verified that our measurements of the axial charge using the fixed source-sink separation method for the three largest values of $\kappa$ agree quantitatively with those calculated by the Wuppertal group using the variable time extent method [14].

On the basis of these and other consistency checks, we are confident that the calculations of moments of quark distributions reported in this work are both reliable and accurate.

\section{Quenched results}

In order to investigate the effects of quenching quantitatively, it is essential to compare quenched and unquenched calculations at equal lattice spacing using precisely the same computational methodology. Hence, we have calculated moments of polarized and unpolarized parton distributions in quenched lattice QCD at $\beta=6.0$, which is comparable to full QCD at $\beta=5.6$. These calculations also afford the opportunity to check our results with the extensive calculations by the QCDSF collaboration [10,15, 52 [55]. For Wilson fermions at $\beta=6.0$, the critical $\kappa$ is $\kappa_{c}=0.1572$, and we have performed our calculations at $\kappa=0.1550,0.1540,0.1530$. Two of these values of $\kappa$ coincide with values used by QCDSF, facilitating direct comparison of unextrapolated as well as extrapolated results. In addition, the pion masses corresponding to these values of $\kappa$ overlap the range of pion masses for the SESAM configurations, providing a meaningful comparison of quenched and unquenched QCD.

Linear extrapolations of the quenched moments of unpolarized up and down quark distributions $\langle x\rangle_{q}^{(b)}$ and $\left\langle x^{2}\right\rangle_{q}$ are shown in Figs. 12 and 13 . Corresponding results for the

quenched moments of polarized quark distributions $\langle 1\rangle_{\Delta q}$, and $\langle x\rangle_{\Delta q}^{(b)}$ are shown in Figs. 14 and 15. The complete numerical data for these and other moments of quark distributions are tabulated in Table $\square$. All results are presented in the $\overline{M S}$ scheme with $\mu^{2}=4 \mathrm{GeV}^{2}$ and extrapolated linearly with a least squares fit to the chiral limit. In these figures, the measured values of $m_{\pi}^{2}$ at each $\kappa$ were converted to physical units using $\frac{1}{a}=2 \mathrm{GeV}$.

Our results for all operators agree within statistics with those of QCDSF, and Figures 12 -15 show typical comparisons. Note that for the three operators that can be evaluated in a zero-momentum proton ground state, $\langle x\rangle_{q}^{(b)},\langle 1\rangle_{\Delta q}$, and $\langle x\rangle_{\Delta q}^{(b)}$ the statistical errors in the extrapolated results are small and allow for meaningful comparison with full QCD results and with phenomenology. In contrast, the errors associated with the operator $\left\langle x^{2}\right\rangle_{q}$, which requires projection onto a state with non-zero momentum, are so large that meaningful comparison will require higher statistics. Since QCDSF used on the order of 1000 configurations whereas we only used 200 configurations, it is consistent that our error bars on individual points are twice as large as theirs for this operator. The fact that the QCDSF error bars are not half the size of ours for three matrix elements calculated in a zero momentum state as well is explained by the fact that the errors they reported for these matrix elements are somewhat larger than jackknife errors because their original analysis used a full error correlation matrix from which low eigenmodes were removed [56. 


\section{E. Full QCD Results}

A central result of this work is the fact that for the range of quark masses accessible in the present calculations, quenched and full QCD calculations at comparable lattice spacing agree within statistical errors. The moments of quark distributions in full QCD at $\beta=5.6$ calculated using SESAM configurations are shown in Table VI. Note that to avoid finite volume effects, moments at the lightest quark mass are not included in the extrapolation. Detailed comparison with Table $\nabla$ shows that in the case of all the operators we have calculated, the full QCD results at $\beta=5.6$ are statistically consistent with those calculated in quenched QCD at $\beta=6.0$. Furthermore, in the case of operators that can be evaluated in zero-momentum nucleon states, the statistical errors are sufficiently small that any differences between the linearly extrapolated quenched and unquenched results are small compared with the discrepancies with experiment discussed below. The level of agreement between quenched and full QCD of $\left\langle x^{2}\right\rangle_{q},\langle 1\rangle_{\Delta q},\langle x\rangle_{\Delta q}^{(b)}$, and $\langle 1\rangle_{\delta q}$ is also shown in Figs 16, 17, 18, and 19 respectively. Hence, the present work strongly rules out the conjecture that the serious discrepancies between quenched calculations and phenomenology arose from the omission of the effect of dynamical quarks. As noted previously, the magnitude of the errors in observables measured in nucleons with non-zero momentum is qualitatively larger, and no strong conclusions can be reached for these observables until higher statistics calculations are carried out.

In addition to the calculations at $\beta=5.6$, which correspond to the same lattice spacing as our quenched calculations, we have also calculated the same operators at $\beta=5.5$ using the SCRI configurations in an attempt to study the approach to the continuum limit. The results are tabulated in Table VII. Note that by Eq. 10, the shifts in $a$ and $\beta$ in going from $\beta=5.6$ to $\beta=5.5$ produce changes in the renormalization constants that are negligible on the scale of the present statistical errors, so these changes in renormalization factors have been omitted. Unfortunately, as pointed out in connection with Fig. 4, the two lightest quark masses lie in a regime in which we expect significant errors due to finite-volume effects, so the chiral extrapolations are physically suspect. Even so, the bulk of the operators evaluated at $\beta=5.5$ and $\beta=5.6$ agree within statistics. The only exceptions, which are only slightly beyond one standard deviation, are $\left\langle x^{2}\right\rangle_{u},\langle 1\rangle_{\Delta u},\langle x\rangle_{\Delta u}^{(b)},\langle 1\rangle_{\delta u}$, and $\langle x\rangle_{\delta d}$. Because of the uncertainty in finite volume effects and small lever arm, we have not attempted to extrapolate the present data to the continuum limit. Higher statistics calculations at smaller quark masses for several coupling constants are presently underway to more seriously address the behavior in the continuum limit.

\section{F. Cooled Results}

A fruitful strategy to obtain insight into hadronic physics is to use the numerical evaluation of the sum over all quark and gluon configurations contributing to the path integral to isolate those paths that dominate the action. In recent years, this approach has provided strong evidence that in QCD with light quarks, topological excitations of the gluon field, which in the semiclassical limit correspond to instantons, play a major role in hadron struc-

ture. By minimizing the action locally in a process known as cooling [57], the instanton content of the quenched and full QCD vacuum has been extracted [58 60]. Comparison of 
hadronic observables calculated with all gluons and those obtained using only the instantons remaining after cooling has demonstrated qualitative agreement for hadron masses, quark distributions, and vacuum correlation functions of hadron currents [61. Calculation of the lowest quark eigenmodes has revealed zero modes correlated spatially with the instantons and truncation of the quark propagators to the zero mode zone has produced the full strength of the $\rho$ and $\pi$ contributions to vacuum correlation functions [62,63].

Hence, to obtain further insight into the structure of the proton, we have used cooling to remove essentially all the gluon fluctuations except for instantons from the full QCD configurations, and compared the quark distributions calculated in full QCD and including only the contributions of instantons. The role of instantons is particularly interesting in considering the spin structure of the proton, since the 't Hooft instanton interaction is the only vertex in QCD that directly removes helicity from valence quarks and transfers it to gluons and quark-antiquark pairs and is therefore a natural mechanism to explain the so-called "spin crisis" 64 66 . Our calculation of the contributions of instantons to matrix elements of operators related by the operator product expansion to deep inelastic scattering also closely parallels the direct calculation of instanton contributions to deep inelastic scattering in refs. 67 70]. The present work extends and substantiates earlier exploratory investigations [12].

To remove the essential non-instanton related gluon fluctuations without producing unnecessary annihilation of instanton-antiinstanton pairs, we have cooled the full QCD SESAM configurations at $\beta=5.6$ using 50 cooling steps. This amount of cooling corresponds to roughly 25 cooling steps for $\beta=5.7$, which was an effective amount of cooling in ref 61. Furthermore, calculations of nucleon two-point functions using the SESAM configurations have shown that the results with 25 and 50 cooling steps differ negligibly [51].

Because of the smoothness of cooled configurations, small statistical errors are obtained using an ensemble of 100 configurations, and previous investigations have shown that the chiral dependence on quark mass is quite linear in the region of interest. Hence, we cooled 100 configurations at $\kappa_{\text {sea }}=0.1570$ and at $\kappa_{\text {sea }}=0.1560$ for our comparison with full QCD. To make the cooled chiral extrapolation comparable to the uncooled case, for each $\kappa_{\text {sea }}$, we selected the cooled valence quark mass, $\kappa_{v a l}$, such that the ratio $\frac{m_{\pi}}{m_{N}}$ was the same as the ratio in full QCD. The resulting cooled values are $\kappa_{\text {val }}=0.1246$ for $\kappa_{\text {sea }}=0.1570$, where $\frac{m_{\pi}}{m_{N}}=0.523$, and $\kappa_{\text {val }}=0.1235$ for $\kappa_{\text {sea }}=0.1560$, where $\frac{m_{\pi}}{m_{N}}=0.476$, and the full QCD masses were taken from ref [16]. Note that, because we are comparing two theoretical calculations in the same physical volume, we have not discarded the lightest quark mass case for full QCD out of concern for finite volume effects, but rather have included it to enable comparison in the regime of the lightest quark masses where dominance by the zero modes associated with instantons should be most pronounced.

Since the high frequency quantum fluctuations are removed by cooling, we set all the renormalization constants, $Z$, to one in order to compare cooled results with the full QCD results. Although we are aware of no rigorous argument as to formulate renormalization in the presence of cooling, this approximation appears the most physical and, for example, is quite sensible for tadpoles, where we expect $U_{0}=\left\langle\frac{1}{3} \operatorname{Tr} U\right\rangle^{1 / 4} \simeq 1$.

The moments of quark distributions calculated in cooled configurations are tabulated in Table VIII. In addition, extrapolations in full and cooled QCD for the operators that can be calculated with high statistics in a nucleon state at zero momentum, $\langle x\rangle_{q}^{(b)},\langle 1\rangle_{\Delta q},\langle x\rangle_{\Delta q}^{(a)}$, 
$\langle 1\rangle_{\delta q}$, and $d_{1}$, are compared in Figs. 20, 21, 22, 23, and 24 respectively.

It is striking that the extrapolated cooled and full QCD results agree so closely for all the twist two operators that correspond to moments of quark distributions. This detailed agreement, generally within error bars but always within two standard deviations, provides strong support for the physical picture that the propagation of light quarks in the nucleon is strongly dominated by the instanton content of the gluon configurations. It is interesting that in the cases of $\langle x\rangle_{q}^{(b)}$ and $\langle x\rangle_{\Delta q}^{(a)}$, the cooled and uncooled results agree best in the region of light quark masses, where we expect zero mode dominance to be most pronounced, and differ much more significantly at heavy quark masses where they have no reason to agree in detail.

Having observed consistency between cooled and full QCD results in the cases above where one expects agreement on the basis of instanton physics, it is also interesting that cooled and full QCD results differ by an order of magnitude for the twist-three operators $d_{1}$ and $d_{2}$ where we expect them to disagree dramatically because of operator mixing. Recall that in connection with Eq 8, we pointed out that for Wilson fermions, the operator

$\gamma_{5} \gamma_{[\sigma} \stackrel{\leftrightarrow}{D}_{\left\{\mu_{1}\right]} \cdots \stackrel{\leftrightarrow}{D}_{\left.\mu_{n}\right\}}$ mixes with the lower dimension operator $\frac{1}{a} \gamma_{5} \gamma_{[\sigma} \gamma_{\left\{\mu_{1}\right]} \cdots \overleftrightarrow{D}_{\left.\mu_{n}\right\}}$. Hence, one expects that our lattice measurements of $d_{1}$ and $d_{2}$ are contaminated to a large extent by operator mixing, and indeed a calculation of the non-perturbative mixing [54 yields a large change in the extracted value of $d_{2}$. As argued above, cooling removes the short wavelength fluctuations responsible for renormalization and mixing, so as a result we would expect the mixing to be reduced essentially to zero. This is precisely what is observed in Fig. 24 and in Table VIII where the cooled measurements extrapolate to values close to zero and the full QCD measurements are an order of magnitude larger.

\section{G. Comparison with Phenomenology}

This section presents the evidence that linear chiral extrapolation of full lattice QCD results in the regime of quark masses accessible in this work is seriously inconsistent with experimental measurements of light cone quark distributions in the nucleon. To make the argument as strong as possible, it is useful to first compare our calculations with three other lattice calculations. Hence, the quenched and full QCD calculations of this work are compared with other related lattice calculations and with phenomenology in Table IX].

We have already commented on the comparison of our quenched calculations with those of the QCDSF collaboration, and their results from refs [10,52 55] are tabulated in the first column to be compared with our quenched results in the fourth column. Here, complementing the plots in Figs 12 - 15, one sees detailed agreement of results calculated at the same $\beta$ and $\kappa$, strongly supporting the accuracy and consistency of both calculations.

The second column shows the extrapolation to the continuum limit by the QCDSF collaboration of several moments [15]. The agreement of these extrapolations with the first column clearly shows that in the case of quenched QCD, finite lattice spacing effects are small compared with the discrepancies with experiment. Given the close agreement between full QCD and quenched calculations in this regime of quark masses, there is no reason to believe that finite lattice spacing effects are substantially larger in our full QCD calculations than in the quenched case, so it would be unreasonable to attribute discrepancies with experiment to finite lattice spacing effects. 
The third column shows the contributions to the axial charge by the SESAM collaboration using the same gluon configurations but completely different measurement technology 14] to be compared with our results in the fifth column. As in the case of the quenched comparisons, we believe this provides additional confirmation of the accuracy and consistency of the present measurements.

The final column shows the moments that were calculated numerically from phenomenological fits to the world supply of deep inelastic lepton scattering data and other high energy scattering data. Data is conveniently accessible on the web from each of the major collaborations, and we have calculated unpolarized moments using each of the unpolarized data sets, CTEQ [1], GRV [2], and MRS [3] and calculated polarized moments using both of the sets GRSV [4], and GS [5]. Unfortunately, it is difficult to provide quantitative estimates of systematic or statistical errors. Experimental data is available only over part of the necessary range of momentum fraction, $x$, and model assumptions are invoked to parameterize parton distributions at large $x$ consistent with known sum rules and physical constraints. In addition, no error correlation matrix is provided, so it is not even possible to calculate the error in a moment that arises from the known statistical errors in the measurements. In order to get some indication of the possible errors, we have calculated the moments using each of the unpolarized or polarized data sets. In the table, we tabulate the average value and give the maximum difference between values for all the relevant data sets in parentheses. Note that these differences are small compared to the statistical errors in the corresponding lattice measurements, so we believe the phenomenological uncertainties are small compared to the discrepancies with lattice extrapolations discussed below. Since, as argued previously, the disconnected diagrams do not contribute to the flavor non-singlet combination $\langle\mathcal{O}\rangle_{u-d}$, we have tabulated the differences between the up and down quark contributions for the relevant combination of $q \pm \bar{q}$ for comparison with the lattice calculations.

Table $\mathbb{X}$ reveals a clear discrepancy between linearly extrapolated lattice calculations and phenomenology. The momentum fraction $\langle x\rangle_{q}$ is a fundamental property specifying the fraction of the total momentum carried by a particular quark flavor. The non-singlet momentum fraction is overestimated by more that 50\%, with full QCD yielding 0.25 0.29 compared with the phenomenological result 0.15 . The second and third moments are similarly overestimated by linear extrapolation.

Another important quantity is the nucleon axial charge, $\langle 1\rangle_{\Delta u-\Delta d}$ governing $\beta$ decay. Here again, one finds major discrepancies with linear chiral extrapolation, with quenched or unquenched calculations yielding 1.0 - 1.15 compared with the experimental value 1.26, corresponding to a discrepancy of 10 to $25 \%$.

Since these and other comparisons in Table [X] show a clear discrepancy between phenomenology and linear chiral extrapolations and because of the evidence summarized previously for the consistency and accuracy of the lattice calculations at heavy quark masses, we believe that the fault lies with the linear chiral extrapolation as discussed in the next section.

\section{H. Chiral Extrapolation}

Superficially, one could imagine that since deep inelastic scattering involves large $Q^{2}$, it might be dominated by short distance behavior of the nucleon wave function and not be 
strongly influenced by the long distance pion cloud. However, as we have seen, the operator product expansion relates moments of structure functions to a tower of local operators to be evaluated in the nucleon ground state. Physically it is clear that the pion cloud should play a major role in the nucleon matrix elements of these operators, and that these contributions are strongly suppressed by the heavy quark masses and small spatial volumes in which we have been forced to work. Whereas there is clearly a heavy quark regime in which matrix elements vary nearly linearly with the quark mass or $m_{\pi}^{2}$, the behavior becomes highly non-linear at quark masses sufficiently light that a substantial pion cloud is produced.

Complementary to the linear heavy quark regime, there is a second regime near the chiral limit in which the behavior is again simple and is specified by chiral perturbation theory. Here, the physics is described by an effective chiral theory based on the would-be Goldstone bosons, and the leading non-analytic behavior can be calculated uniquely. For example, of direct relevance to this work, the leading non-analytic behavior of $\left\langle x^{n}\right\rangle_{u-d}$ is 71.72

$$
\left\langle x^{n}\right\rangle_{u-d} \sim a_{n}\left[1-\frac{\left(3 g_{A}^{2}+1\right) m_{\pi}^{2}}{\left(4 \pi f_{\pi}\right)^{2}} \ln m_{\pi}^{2}\right] .
$$

Unfortunately, at present, there is no full analytical theory joining the chiral regime with the heavy quark regime of the present lattice calculation. Hence to explore the chiral extrapolation of lattice QCD calculations to the physical region, it is useful to use a physically motivated extrapolation formula incorporating the correct behavior in the chiral limit. We therefore fit the lattice data with the extrapolation formula of Ref. [18], in which a phenomenological cutoff $\mu$ is introduced in the nonanalytic term to specify the size of the source generating the pion cloud and the usual analytic term in $m_{\pi}^{2}$ is included:

$$
\left\langle x^{n}\right\rangle_{u-d} \sim a_{n}\left[1-\frac{\left(3 g_{A}^{2}+1\right) m_{\pi}^{2}}{\left(4 \pi f_{\pi}\right)^{2}} \ln \left(\frac{m_{\pi}^{2}}{m_{\pi}^{2}+\mu^{2}}\right)\right]+b_{n} m_{\pi}^{2} .
$$

Physically, it is reasonable that momenta in the pion loop should not become infinitely large as they would in the presence of a point source, but rather be cut off at a scale characteristic of the size of the valence quark core of the nucleon, and alternative parameterizations in terms of a form factor give equivalent results.

The result of using this extrapolation formula for the difference between the up and down quark momentum fraction $\left\langle x^{n}\right\rangle_{u-d}$ is shown in Fig. 25. Although this extrapolation is only valid in full QCD, since full QCD and quenched results are equivalent in the regime of our calculations, to improve statistics, we have also included our quenched results. The heavy solid curve is the result of a least-squares fit of $a_{1}$ and $b_{1}$ in Eq. 21 with fixed $\mu=550 \mathrm{MeV}$ and the the light solid lines indicate the jackknife error band. Here we see clearly that the extrapolation formula containing the leading chiral behavior is consistent both with the lattice measurements and the experimental data. The fact that the effect of the chiral logarithm is much larger in this matrix element than in more familiar mass measurements is again a manifestation of the variational theorem. Making an order $\epsilon$ error in the pion cloud of the wave function makes an order $\epsilon$ error in matrix elements of general operators but only an order $\epsilon^{2}$ error in the mass. The obvious problem with the present argument is that although a cutoff of order $0.3 \mathrm{fm}$ is physically reasonable, it has not been calculated from first principles and may be regarded as a single free parameter introduced to fit a single experimental measurement. 
To see that this single cutoff parameter resolves the discrepancy with experiment for a number of observables, the results of using Eq. 21 for the first three moments of the difference between the up and down quark density distributions $\left\langle x^{n}\right\rangle_{u-d}$ are shown in figure 26. Because of the larger error bars in the higher moments, we have also included the quenched QCDSF results [10] to provide a larger lever arm in the least squares fit. As seen in the figure, the single value of $\mu$ provides simultaneous agreement with experiment for all three moments. As shown in Ref. [18], this strong chiral behavior of the three lowest moments is also observed in chiral bag models. Furthermore, similar extrapolation with a comparable cutoff also reconciles the strong discrepancy between linear extrapolation of lattice results and experimental nucleon magnetic moments [17]. Thus, a consistent picture is emerging concerning the importance of large effects of the pion cloud in chiral extrapolations. Unfortunately, because spin-dependent structure functions also involve significant contributions from Delta excitations and chiral perturbation theory becomes less well controlled, we do not presently have a corresponding physical interpolation formula for $\left\langle x^{n}\right\rangle_{\Delta u-\Delta d}$.

The curves in Fig. 26 suggest that precision measurements down to $m_{\pi}^{2}=0.05 \mathrm{GeV}^{2}$ are required to determine the parameters of the chiral extrapolation and thereby provide reliable extrapolation of moments of quark distributions. The computational resources required for such calculations may be estimated using the cost function [73] obtained by the SESAM collaboration, which provided the gluon configurations. For present purposes, the number of floating point operations per independent gluon configuration, $N$ in Teraflops-years, may be conveniently written

$$
N \simeq .038\left[\frac{L}{4}\right]^{4.55}\left[\frac{.08}{a}\right]^{7.25}\left[\frac{.3}{\frac{m_{\pi}}{m_{\rho}}}\right]^{2.7} .
$$

Because the spatial derivatives and non-zero momentum projections required to calculate moments of structure functions require high Monte Carlo statistics, it is necessary to calculate of the order of 400 independent configurations. Including equilibration and calculation at higher quark masses, the total computer time is approximately twice that required for 400 configurations at the lowest quark mass. Hence, a calculation with a lattice spacing

$a=0.1 \mathrm{fm}$ and $\frac{m_{\pi}}{m_{\rho}}=0.3$ would require approximately 8 Teraflops-years, i.e., dedicated use of a computer that sustains 8 Teraflops on QCD for one year. Such resources should become available in the next generation of lattice QCD computers.

\section{SUMMARY AND CONCLUSIONS}

This work has presented the first calculation of the moments of light cone quark distributions in full QCD. The methodology has been presented in detail and validated by numerous consistency checks and comparison with other relevant lattice calculations.

One major result of this work is the close agreement of full QCD and quenched calculations for quark masses corresponding to $m_{\pi}$ above $500 \mathrm{MeV}$. This agreement rules out the conjecture that discrepancies with experiment could be attributed to quenching effects.

A second salient result is clear evidence that linear extrapolation of full QCD lattice results from pion masses above $500 \mathrm{MeV}$ is in serious disagreement with experiment, ranging from the order of $50 \%$ for the quark momentum fraction to $10-25 \%$ for the axial charge. 
Motivated by the inadequacy of linear chiral extrapolation, we have shown that extrapolation including the leading nonanalytic behavior of chiral perturbation theory has the potential to yield results consistent with phenomenology. We have explicitly shown that an extrapolation formula with a single phenomenological cutoff simultaneously fits the first three moments of the quark momentum fraction.

Finally, we have shown qualitative agreement between full QCD and cooled lattice configurations retaining only the contributions of instantons, providing additional evidence for the role of instantons in light hadron structure and of zero-mode dominance.

This work points the way for a number of promising steps in our continued quest to understand hadron structure from first principles using lattice QCD. One should clearly undertake a systematic program of using a partially quenched chiral expansion for extrapolation and measure the relevant parameters of the effective chiral theory. To complement the flavor non-singlet matrix elements of this work, we need to calculate the disconnected diagrams required to compare flavor singlet matrix elements with phenomenology. The continuum limit of the SESAM results should be explored by supplementing the existing SCRI configurations with additional quark masses so that calculations at $\beta=5.6,5.5$, and 5.3 may be compared and extrapolated. It is desirable to undertake nonperturbative renormalization without Gribov ambiguities associated with gauge fixing, and to this end we note that the Schrödinger functional method has now been used to calculate the average quark momentum in the pion [35, 76 78]. Quenched calculations with chiral fermions in a spatial volume of $3.2 \mathrm{fm}$ should be carried out to extend the pion mass down to $250 \mathrm{MeV}$ and to remove the problem of operator mixing in the twist-3 matrix elements. Finite volume formulae for operator matrix elements should be derived to correct residual finite volume effects. As lattice results come closer to phenomenology, it will be important to have quantitative understanding of the errors in moments of structure functions arising from systematic errors, as well as the error correlation matrix describing statistical errors. Finally, with the advent of multi-Terascale computers, the promise of full QCD calculations with light pion masses will finally be within our grasp.

We note that subsequent to the completion of this work, a thorough analysis of the error correlations in the experimental measurements of polarized parton distributions was performed in ref [79], with the result that the errors in the phenomenological values of moments of $\Delta u-\Delta d$ in table $\mathbb{X}$ are of the order of ten percent.

\section{ACKNOWLEDGMENTS}

The authors wish to thank R.Horsley, W. Melnitchouk, Edward Shuryak, and A. W. Thomas for helpful discussions and communications. The research in this work was supported in part by the U.S. Department of Energy under cooperative research agreement DEFC02-94ER40818 and contracts DE-FG02-91ER40676 and DE-FG02-97ER41022. R. G. E. was supported by DOE contract DE-AC05-84ER40150 under which the Southeastern Universities Research Association (SURA) operates the Thomas Jefferson National Accelerator Facility (TJNAF). S. C., Th. L., and K. S. acknowledge the EU Network HPRN-CT-200000145 "Hadron Phenomenology from Lattice QCD" for support. We gratefully acknowledge use of computer resources provided by the MIT and Jefferson Lab LHPC clusters, the SCRI CM-2, and the Jlab QCDSP. We thank the John von Neumann-Institute for Computing for 
providing the Cray T3E and APE100 facilities and the staff of the computer centers ZAM at FZ-Jlich and DESY/Zeuthen for their enormous support.

\section{APPENDIX A: CALCULATION OF SEQUENTIAL PROPAGATORS}

This appendix describes the explicit calculation of the sequential nucleon sources used in the calculations in this work.

Denote the smeared field by $\Psi$, the original unsmeared field by $\psi$ and the gauge invariant smearing function defined by Eq. 13 by $F$ so that

$$
\Psi_{\alpha}^{a}\left(\vec{x}_{0}, t_{0}\right)=\int d^{3} x F_{\alpha \alpha^{\prime}}^{a a^{\prime}}\left(\vec{x}_{0}, \vec{x}\right) \psi_{\alpha^{\prime}}^{a^{\prime}}\left(\vec{x}, t_{0}\right)
$$

The forward propagator from a smeared source to a point sink, denoted

$$
S_{\alpha \alpha^{\prime}}^{a a^{\prime}}\left(\psi \Psi \mid \vec{x}, t ; \vec{x}_{0}, t_{0}\right) \stackrel{\text { def }}{=}\left\langle\psi_{\alpha}^{a}(\vec{x}, t) \bar{\Psi}_{\alpha^{\prime}}^{a^{\prime}}\left(\vec{x}_{0}, t_{0}\right)\right\rangle,
$$

is the solution to the linear system

$$
\int d t^{\prime} d^{3} x^{\prime} D_{W \alpha \alpha^{\prime \prime}}^{a a^{\prime \prime}}\left(\vec{x}, t ; \vec{x}^{\prime}, t^{\prime}\right) S_{\alpha^{\prime \prime} \alpha^{\prime}}^{a^{\prime \prime} a^{\prime}}\left(\psi \Psi \mid \vec{x}^{\prime}, t^{\prime} ; \vec{x}_{0}, t_{0}\right)=\delta\left(t-t_{0}\right) F_{\alpha \alpha^{\prime}}^{a a^{\prime}}\left(\vec{x}, \vec{x}_{0}\right),
$$

where $D_{W}$ denotes the Wilson Dirac operator and the smearing function is the source term. The propagator from a smeared source to a smeared sink is obtained by an additional smearing,

$$
\begin{aligned}
S_{\alpha \alpha^{\prime}}^{a a^{\prime}}\left(\Psi \Psi \mid \vec{x}, t ; \vec{x}_{0}, t_{0}\right) & \stackrel{\text { def }}{=}\left\langle\Psi_{\alpha}^{a}(\vec{x}, t) \bar{\Psi}_{\alpha^{\prime}}^{a^{\prime}}\left(\vec{x}_{0}, t_{0}\right)\right\rangle \\
& =\int d^{3} x^{\prime} F_{\alpha \alpha^{\prime \prime}}^{a a^{\prime \prime}}\left(\vec{x}, \vec{x}^{\prime}\right) S_{\alpha^{\prime \prime} \alpha^{\prime}}^{a^{\prime \prime} a^{\prime}}\left(\psi \Psi \mid \vec{x}^{\prime}, t ; \vec{x}_{0}, t_{0}\right) .
\end{aligned}
$$

The three point function specifying $u$ quark operator matrix elements, using the proton current, Eq. 12, with smeared quark fields, may be written:

$$
\begin{aligned}
& \left\langle J^{\alpha} O \bar{J}^{\alpha^{\prime}}\right\rangle^{(u)}\left(t_{i}, t_{f}, t_{o}, \vec{x}_{i}\right)=\int d^{3} x_{o} \int d^{3} x_{f} e^{i \vec{p} \vec{x}_{f}} \epsilon^{a b c} \epsilon^{a^{\prime} b^{\prime} c^{\prime}} \Gamma_{\beta \gamma} \bar{\Gamma}_{\beta^{\prime} \gamma^{\prime}} \times \\
& \left\langle U_{\alpha}^{a}\left(\vec{x}_{f}, t_{f}\right) U_{\beta}^{b}\left(\vec{x}_{f}, t_{f}\right) D_{\gamma}^{c}\left(\vec{x}_{f}, t_{f}\right) \bar{u}_{\nu}^{d}\left(\vec{x}_{o}, t_{o}\right) O_{\nu \nu^{\prime}}^{d d^{\prime}} u_{\nu^{\prime}}^{d^{\prime}}\left(\vec{x}_{o}^{\prime}, t_{o}^{\prime}\right) \bar{U}_{\alpha^{\prime}}^{a^{\prime}}\left(\vec{x}_{i}, t_{i}\right) \bar{U}_{\beta^{\prime}}^{b^{\prime}}\left(\vec{x}_{i}, t_{i}\right) \bar{D}_{\gamma^{\prime}}^{c^{\prime}}\left(\vec{x}_{i}, t_{i}\right)\right\rangle
\end{aligned}
$$

where $t_{i}, t_{f}$ and $t_{o}$ are time coordinates of the source, sink and operator insertion, $u$ and $U$ denote point and smeared fields for the $u$-quark, $d$ and $D$ denote point and smeared fields for the $d$-quark, and $\Gamma=C \gamma_{5}$. The three point function for a $d$ quark operator has the same form with the operator insertion $\bar{d}_{\nu}^{d}\left(\overrightarrow{x_{o}}, t_{o}\right) O_{\nu \nu^{\prime}}^{d d^{\prime}} d_{\nu^{\prime}}^{d^{\prime}}\left(\vec{x}_{o}^{\prime}, t_{o}^{\prime}\right)$.

After performing the connected diagram contractions, the $u$ and $d$ operator matrix elements may be written:

$$
\begin{aligned}
& \left\langle J^{\alpha} O \bar{J}^{\alpha^{\prime}}\right\rangle^{(u, d)}\left(t_{i}, t_{f}, t_{o}\right)=\int d^{3} x_{o} \int d^{3} x_{f} e^{i \vec{p} \vec{x}_{f}} \mathcal{M}_{\mu \mu^{\prime}}^{(u, d)\left[\alpha \alpha^{\prime}\right] a a^{\prime}}\left(\vec{x}_{f}, t_{f}, \vec{x}_{i}, t_{i}\right) \\
& \times S_{\mu \nu}^{a d}\left(\Psi \psi \mid \vec{x}_{f}, t_{f} ; \vec{x}_{o}, t_{o}\right) O_{\nu \nu^{\prime}}^{d d^{\prime}} S_{\nu^{\prime} \mu^{\prime}}^{d^{\prime}{ }^{\prime}}\left(\psi \Psi \mid \vec{x}_{o}^{\prime}, t_{o}^{\prime}, \vec{x}_{i}, t_{i}\right)
\end{aligned}
$$

where 


$$
\begin{aligned}
& \mathcal{M}_{\mu \mu^{\prime}}^{(u)\left[\alpha \alpha^{\prime}\right] a a^{\prime}}\left(\vec{x}_{f}, t_{f}, t_{i}\right) \stackrel{\text { def }}{=} \epsilon^{a b c} \epsilon^{a^{\prime} b^{\prime} c^{\prime}} S_{\gamma \gamma^{\prime}}^{c c^{\prime}} \times \\
& \quad \times\left[S_{\alpha \alpha^{\prime}}^{b b^{\prime}} \Gamma_{\mu \gamma} \bar{\Gamma}_{\mu^{\prime} \gamma^{\prime}}+S_{\alpha \beta^{\prime}}^{b b^{\prime}} \Gamma_{\mu \gamma} \bar{\Gamma}_{\beta^{\prime} \gamma^{\prime}} \delta_{\mu^{\prime} \alpha^{\prime}}+S_{\beta \alpha^{\prime}}^{b b^{\prime}} \Gamma_{\beta \gamma} \bar{\Gamma}_{\mu^{\prime} \gamma^{\prime}} \delta_{\mu \alpha}+S_{\beta \beta^{\prime}}^{b b^{\prime}} \Gamma_{\beta \gamma} \bar{\Gamma}_{\beta^{\prime} \gamma^{\prime}} \delta_{\mu \alpha} \delta_{\mu^{\prime} \alpha^{\prime}}\right], \\
& \mathcal{M}_{\mu \mu^{\prime}}^{(d)\left[\alpha \alpha^{\prime}\right] a a^{\prime}}\left(\vec{x}_{f}, t_{f}, t_{i}\right) \stackrel{\text { def }}{=} \epsilon^{a b c} \epsilon^{a^{\prime} b^{\prime} c^{\prime}}\left[S_{\alpha \alpha^{\prime}}^{b b^{\prime}} S_{\beta \beta^{\prime}}^{c c^{\prime}}+S_{\alpha \beta^{\prime}}^{b b^{\prime}} S_{\beta \alpha^{\prime}}^{c c c^{\prime}}\right] \Gamma_{\beta \mu} \Gamma_{\beta^{\prime} \mu^{\prime}},
\end{aligned}
$$

and $S_{\mu \mu^{\prime}}^{a a^{\prime}} \equiv S_{\mu \mu^{\prime}}^{a a^{\prime}}\left(\Psi \Psi \mid \vec{x}_{f}, t_{f}, \vec{x}_{i}, t_{i}\right)$.

We now define the backward propagator

$$
\mathcal{B}_{\nu^{\prime \prime} \mu^{\prime}}^{\left[\alpha \alpha^{\prime}\right] d a^{\prime}}\left(\vec{x}_{o}, t_{o}, t_{f}, \vec{x}_{i}, t_{i}\right) \stackrel{\text { def }}{=} \int d^{3} x_{f} e^{-i \vec{p} \vec{x}_{f}} S_{\nu^{\prime \prime} \mu^{\prime \prime}}^{d a}\left(\psi \Psi \mid \vec{x}_{o}, t_{o} ; \vec{x}_{f}, \vec{x}_{i}, t_{f}\right) \gamma_{\mu^{\prime \prime} \mu}^{5} \mathcal{M}_{\mu \mu^{\prime}}^{*\left[\alpha \alpha^{\prime}\right] a a^{\prime}}\left(\vec{x}_{f}, t_{f}, t_{i}\right) .
$$

Since it propagates from the sink to the operator, we use the relation $\gamma^{5} S(x, y) \gamma^{5}=$ $S^{\dagger}(y, x)$ to obtain

$$
\left[\int d^{3} x_{f} e^{i \vec{p} \vec{x}_{f}} \mathcal{M}_{\mu \mu^{\prime}}^{\left[\alpha \alpha^{\prime}\right] a a^{\prime}}\left(\vec{x}_{f}, t_{f}, \vec{x}_{i}, t_{i}\right) S_{\mu \nu}^{a d}\left(\Psi \psi \mid \vec{x}_{f}, t_{f} ; \vec{x}_{o}, t_{o}\right)\right]^{*}=\gamma_{\nu \nu^{\prime \prime}}^{5} \mathcal{B}_{\nu^{\prime \prime} \mu^{\prime}}^{\left[\alpha \alpha^{\prime}\right] d a^{\prime}}\left(\vec{x}_{o}, t_{o}, t_{f}, \vec{x}_{i}, t_{i}\right)
$$

so that (A5) becomes

$$
\left\langle J^{\alpha} O J^{\alpha^{\prime}}\right\rangle\left(t_{i}, t_{f}, t_{o}\right)=\int d^{3} x_{o} \gamma_{\nu \nu^{\prime \prime}}^{5} \mathcal{B}_{\nu^{\prime \prime} \mu^{\prime}}^{*\left[\alpha \alpha^{\prime}\right] d a^{\prime}}\left(\vec{x}_{o}, t_{o}, t_{f}, \vec{x}_{i}, t_{i}\right) O_{\nu \nu^{\prime}}^{d d^{\prime}} S_{\nu^{\prime} \mu^{\prime}}^{d^{\prime} a^{\prime}}\left(\psi \Psi \mid \vec{x}_{o}, t_{o}, \vec{x}_{i}, t_{i}\right) .
$$

As in Eq. (A2), the backward propagator $\mathcal{B}^{\left[\alpha \alpha^{\prime}\right]}$ defined by (A8) may be calculated by solving the linear system:

$$
\begin{aligned}
\int d t^{\prime} d^{3} x^{\prime} & D_{W}^{a d \mu^{\prime \prime} \nu^{\prime \prime}} \\
& =\delta\left(t-t_{f}\right) e^{-i \vec{x} \vec{x}} \int d^{3} x^{\prime} F_{\mu^{\prime \prime} \rho}^{a a^{\prime \prime}}\left(\vec{x}, \vec{x}^{\prime}\right) \gamma_{\rho \mu}^{5} \mathcal{M}_{\mu \mu^{\prime}}^{*\left[\alpha \alpha^{\prime}\right] a^{\prime \prime} a^{\prime}}\left(\vec{x}^{\prime}, t_{f}\right) .
\end{aligned}
$$

Finally, in the same notation, the momentum projected two-point function with smeared sources is:

$$
\begin{aligned}
\left\langle J^{\alpha} \bar{J}^{\alpha^{\prime}}\right\rangle\left(t_{i}, t_{f}, \vec{x}_{i}\right)= & \int d^{3} x_{f} e^{i \vec{p} \vec{x}_{f}} \epsilon^{a b c} \epsilon^{a^{\prime} b^{\prime} c^{\prime}} \Gamma_{\beta \gamma} \bar{\Gamma}_{\beta^{\prime} \gamma^{\prime}} \times \\
& \left\langle U_{\alpha}^{a}\left(\vec{x}_{f}, t_{f}\right) U_{\beta}^{b}\left(\vec{x}_{f}, t_{f}\right) D_{\gamma}^{c}\left(\vec{x}_{f}, t_{f}\right) \bar{U}_{\alpha^{\prime}}^{a^{\prime}}\left(\vec{x}_{i}, t_{i}\right) \bar{U}_{\beta^{\prime}}^{b^{\prime}}\left(\vec{x}_{i}, t_{i}\right) \bar{D}_{\gamma^{\prime}}^{c^{\prime}}\left(\vec{x}_{i}, t_{i}\right)\right\rangle \\
= & \int d^{3} x_{f} e^{i \vec{p} \vec{x}_{f}} \epsilon^{a b c} \epsilon^{a^{\prime} b^{\prime} c^{\prime}} \Gamma_{\beta \gamma} \bar{\Gamma}_{\beta^{\prime} \gamma^{\prime}} S_{\gamma \gamma^{\prime}}^{c c^{\prime}}\left[S_{\alpha \alpha^{\prime}}^{a a^{\prime}} S_{\beta \beta^{\prime}}^{b b^{\prime}}-S_{\alpha \beta^{\prime}}^{a b^{\prime}} S_{\beta \alpha^{\prime}}^{b a^{\prime}}\right] .
\end{aligned}
$$




\section{REFERENCES}

[1] H. L. Lai et al., Phys. Rev. D 55, 1280 (1997) hep-ph/9606399. Data provided at http://www-spires.dur.ac.uk/hepdata/cteq.htm

[2] M. Gluck, E. Reya and A. Vogt, Eur. Phys. J. C 5, 461 (1998) arXiv:hep-ph/9806404. Data provided at http://www-spires.dur.ac.uk/hepdata/grv.htm

[3] A. D. Martin, R. G. Roberts, W. J. Stirling and R. S. Thorne, arXiv:hep-ph/0110215. Data provided at http://durpdg.dur.ac.uk/hepdata/mrs.htm

[4] M. Gluck, E. Reya, M. Stratmann and W. Vogelsang, Phys. Rev. D 53, 4775 (1996) arXiv:hep-ph/9508347. Data provided at http://doom.physik.uni-dortmund.de/PARTON/index.htm]

[5] T. Gehrmann and W. J. Stirling, Phys. Rev. D 53, 6100 (1996) arXiv:hep-ph/9512406. Data provided at http://www.desy.de/ gehrt/pdf

[6] D. Adams et al. [Spin Muon Collaboration (SMC)], Phys. Rev. D 56, 5330 (1997) hepex/9702005].

[7] B. Adeva et al. [Spin Muon Collaboration (SMC)], Phys. Lett. B 420, 180 (1998) hep$\mathrm{ex} / 9711008$.

[8] K. Ackerstaff et al. [HERMES Collaboration], Phys. Lett. B 464, 123 (1999) hepex/9906035.

[9] D. Dolgov et al., Nucl. Phys. Proc. Suppl. 94, 303 (2001) arXiv:hep-lat/0011010.

[10] M. Göckeler, R. Horsley, E. M. Ilgenfritz, H. Perlt, P. Rakow, G. Schierholz and A. Schiller, Phys. Rev. D 53, 2317 (1996) arXiv:hep-lat/9508004.

[11] C. Best et al., hep-ph/9706502.

[12] D. Dolgov, R. Brower, J. W. Negele and A. Pochinsky, Nucl. Phys. Proc. Suppl. 73, 300 (1999) arXiv:hep-lat/9809132.

[13] S.J. Dong, J.-F. Lagae, K.F. Liu, Phys. Rev. Lett. 75 2096-2099 (1995).

[14] S. Gusken et al. [SESAM Collaboration], arXiv:hep-lat/9901009.

[15] S. Capitani et al., Nucl. Phys. (Proc. Suppl.) B79, 548 (1999) hep-ph/9905573.

[16] N. Eicker et al. [SESAM collaboration], Phys. Rev. D 59, 014509 (1999) arXiv:heplat/9806027].

[17] D. B. Leinweber, D. H. Lu and A. W. Thomas, Phys. Rev. D 60, 034014 (1999) arXiv:hep-lat/9810005.

[18] W. Detmold, W. Melnitchouk, J. W. Negele, D. B. Renner and A. W. Thomas, Phys. Rev. Lett. 87, 172001 (2001) arXiv:hep-lat/0103006.

[19] R. L. Jaffe and X. Ji, Nucl. Phys. B 375, 527 (1992).

[20] S. Wandzura and F. Wilczek, Phys. Lett. B 72, 195 (1977).

[21] S. Capitani et al., Nucl. Phys. (Proc. Suppl.) B63, 871 (1998) arXiv:hep-lat/9710034.

[22] M. Gockeler et al., Phys. Rev. D 63, 074506 (2001) arXiv:hep-lat/0011091.

[23] S. Capitani, Nucl. Phys. B 597, 313 (2001) arXiv:hep-lat/0009018].

[24] J. E. Mandula, G. Zweig and J. Govaerts, Nucl. Phys. B 228, 91 (1983).

[25] G. Beccarini, M. Bianchi, S. Capitani and G. Rossi, Nucl. Phys. B 456, 271 (1995) arXiv:hep-lat/9506021.

[26] M. Göckeler, R. Horsley, E. M. Ilgenfritz, H. Perlt, P. Rakow, G. Schierholz and A. Schiller, Nucl. Phys. B 472, 309 (1996) arXiv:hep-lat/9603006.

[27] M. Göckeler, R. Horsley, E. M. Ilgenfritz, H. Perlt, P. Rakow, G. Schierholz and A. Schiller, Phys. Rev. D 54, 5705 (1996) arXiv:hep-lat/9602029. 
[28] S. Capitani, M. Gockeler, R. Horsley, H. Perlt, P. E. Rakow, G. Schierholz and A. Schiller, Nucl. Phys. B 593, 183 (2001) arXiv:hep-lat/0007004.

[29] A. S. Kronfeld and D. M. Photiadis, Phys. Rev. D 31, 2939 (1985).

[30] S. Capitani, Nucl. Phys. B 592, 183 (2001) arXiv:hep-lat/0005008.

[31] S. Capitani and G. Rossi, Nucl. Phys. B 433, 351 (1995) arXiv:hep-lat/9401014.

[32] R. C. Brower, S. Huang, J. W. Negele, A. Pochinsky and B. Schreiber, Nucl. Phys. Proc. Suppl. 53, 318 (1997) arXiv:hep-lat/9608069.

[33] D. Dolgov Ph.D. thesis, MIT, 2000.

[34] H. Neff, N. Eicker, T. Lippert, J. W. Negele and K. Schilling, Phys. Rev. D 64, 114509 (2001) arXiv:hep-lat/0106016.

[35] M. Guagnelli, K. Jansen and R. Petronzio, Phys. Lett. B 493, 77 (2000) arXiv:heplat/0009006.

[36] J. M. Grandy Ph.D. thesis, MIT, 1992.

[37] A. Pochinsky Ph.D. thesis, MIT,1997.

[38] C. Alexandrou, F. Jegerlehner, S. Güsken, K. Schilling and R. Sommer, Phys. Lett. B 256, 60 (1991).

[39] C. R. Allton et al. [UKQCD Collaboration], Phys. Rev. D 47, 5128 (1993) arXiv:heplat/9303009.

[40] A. Frommer, B. Nockel, S. Gusken, T. Lippert and K. Schilling, Int. J. Mod. Phys. C 6, 627 (1995) arXiv:hep-lat/9504020.

[41] M. Fukugita, Y. Kuramashi, M. Okawa, A. Ukawa, Phys.Rev. Lett. 75 2092-2095 (1995).

[42] S. Aoki, M. Doui, T. Hatsuda, Y. Kuramashi, Phys. Rev. D 56 433-436 (1997).

[43] S. Güsken et al. [SESAM Collaboration], Phys. Rev. D 59, 054504 (1999) arXiv:heplat/9809066].

[44] L. Maiani, G. Martinelli, M. L. Paciello and B. Taglienti, Nucl. Phys. B 293, 420 (1987).

[45] C. Bernard, T. Draper, G. Hockney and A. Soni, In *Wuppertal 1985, Proceedings, Lattice Gauge Theory*, 199 (1985).

[46] B. Efron, "The Jackknife, the Bootstrap and Other Resampling Plans" CBMS Press, 1982.

[47] "Numerical Recipes in C," Academic Press, 1995.

[48] K. M. Bitar, R. G. Edwards, U. M. Heller and A. D. Kennedy, Nucl. Phys. (Proc. Suppl.) B53, 225 (1997) arXiv:hep-lat/9607043.

[49] B. Orth, private communication.

[50] M. Luscher and P. Weisz, JHEP 0109, 010 (2001) [arXiv:hep-lat/0108014.

[51] V. H. Martinez, Ph.D. thesis, MIT, 2000.

[52] M. Göckeler, R. Horsley, L. Mankiewicz, H. Perlt, P. Rakow, G. Schierholz and A. Schiller, Phys. Lett. B 414, 340 (1997) hep-ph/9708270.

[53] M. Göckeler, R. Horsley, H. Perlt, P. Rakow, G. Schierholz, A. Schiller and P. Stephenson, hep-ph/9711245.

[54] M. Göckeler, R. Horsley, W. Kürzinger, H. Oelrich, P. Rakow and G. Schierholz, hepph/9909253.

[55] M. Gockeler et al., Phys. Rev. D 63, 074506 (2001) arXiv:hep-lat/0011091.

[56] R. Horsley, private communication.

[57] B. Berg, Phys. Lett. B 104475 (1981).

[58] J. W. Negele, Nucl. Phys. Proc. Suppl. 73, 92 (1999) arXiv:hep-lat/9810053. 
[59] T. L. Ivanenko and J. W. Negele, Nucl. Phys. Proc. Suppl. 63, 504 (1998) arXiv:heplat/9709130|.

[60] A. Ringwald and F. Schrempp, Phys. Lett. B 459, 249 (1999) arXiv:hep-lat/9903039.

[61] M. C. Chu, J. M. Grandy, S. Huang and J. W. Negele, Phys. Rev. D 49, 6039 (1994) arXiv:hep-lat/9312071.

[62] T. L. Ivanenko, Ph.D. thesis, MIT, 1997.

[63] J. W. Negele, Nucl. Phys. A 670, 14 (2000) [arXiv:hep-lat/9902032].

[64] S. Forte and E. V. Shuryak, Nucl. Phys. B 357, 153 (1991).

[65] G. M. Shore and G. Veneziano, Mod. Phys. Lett. A 8, 373 (1993).

[66] N. I. Kochelev, Phys. Rev. D 57, 5539 (1998) arXiv:hep-ph/9711226.

[67] A. Ringwald and F. Schrempp, HERA," arXiv:hep-ph/9411217.

[68] S. Moch, A. Ringwald and F. Schrempp, Nucl. Phys. B 507, 134 (1997) arXiv:hep$\mathrm{ph} / 9609445]$.

[69] A. Ringwald and F. Schrempp, Phys. Lett. B 438, 217 (1998) arXiv:hep-ph/9806528.

[70] A. Ringwald and F. Schrempp, Phys. Lett. B 503, 331 (2001) arXiv:hep-ph/0012241.

[71] J. W. Chen and X. D. Ji, arXiv:hep-ph/0105197.

[72] D. Arndt and M. J. Savage, arXiv:nucl-th/0105045.

[73] T. Lippert, S. Gusken and K. Schilling, Nucl. Phys. Proc. Suppl. 83, 182 (2000).

[74] R. Gupta, C. F. Baillie, R. G. Brickner, G. W. Kilcup, A. Patel and S. R. Sharpe, Phys. Rev. D 44, 3272 (1991).

[75] K. M. Bitar et al., Phys. Rev. D 49, 3546 (1994) arXiv:hep-lat/9309011].

[76] K. Jansen, arXiv:hep-lat/0010038.

[77] M. Guagnelli, K. Jansen and R. Petronzio, Phys. Lett. B 457, 153 (1999) arXiv:heplat/9901016.

[78] M. Guagnelli, K. Jansen and R. Petronzio, Nucl. Phys. B 542, 395 (1999) arXiv:heplat/9809009.

[79] J. Blumlein and H. Bottcher, arXiv:hep-ph/0203155. 


\section{TABLES}

\begin{tabular}{|c|c|c|c|c|c|c|c|}
\hline Data set & QCD & $\overline{L_{x}^{3} \times L_{t}}$ & $\beta$ & $\kappa_{\text {sea }}$ & $\kappa_{v a l}$ & $\begin{array}{l}\text { Approx. } \\
\text { \# configs. }\end{array}$ & $\begin{array}{l}\text { Trajectory } \\
\text { separation }\end{array}$ \\
\hline \multirow[t]{4}{*}{$\overline{\text { SESAM }}$} & full & $16^{3} \times 32$ & 5.6 & 0.1560 & 0.1560 & 200 & 25 \\
\hline & & & & 0.1565 & 0.1565 & 200 & 25 \\
\hline & & & & 0.1570 & 0.1570 & 200 & 25 \\
\hline & & & & 0.1575 & 0.1575 & 200 & 25 \\
\hline \multirow[t]{3}{*}{ SCRI } & full & $16^{3} \times 32$ & 5.5 & 0.1596 & 0.1596 & 100 & 20 \\
\hline & & & & 0.1600 & 0.1600 & 100 & 20 \\
\hline & & & & 0.1604 & 0.1604 & 100 & 18 \\
\hline \multirow[t]{3}{*}{ MIT } & quenched & $16^{3} \times 32$ & 6.0 & $m_{q}=\infty$ & 0.1530 & 200 & \\
\hline & & & & & 0.1540 & 200 & \\
\hline & & & & & 0.1550 & 200 & \\
\hline \multirow[t]{2}{*}{ SESAM-cooled } & cooled & $16^{3} \times 32$ & 5.6 & 0.1560 & 0.1235 & 100 & \\
\hline & & & & 0.1570 & 0.1246 & 100 & \\
\hline
\end{tabular}

TABLE I. Parameters specifying the full QCD, quenched, and cooled gluon configurations used in calculations of moments of quark distributions. For full QCD calculations, the number of hybird Monte Carlo trajectories between measurements is given in the last column. 


\begin{tabular}{|c|c|c|c|c|}
\hline observable & $\mathrm{H}(4)$ & mixing & $\vec{P}$ & lattice operator \\
\hline$\overline{\langle x\rangle_{q}^{(a)}}$ & $6_{3}^{+}$ & no & 1 & $\bar{q} \gamma_{\{1} \stackrel{\leftrightarrow}{D}_{4\}} q$ \\
\hline$\langle x\rangle_{q}^{(b)}$ & $\mathbf{3}_{1}^{+}$ & no & 0 & $\bar{q} \gamma_{4} \stackrel{\leftrightarrow}{D}_{4} q-\frac{1}{3}\left(\bar{q} \gamma_{1} \stackrel{\leftrightarrow}{D}_{1} q+\bar{q} \gamma_{2} \stackrel{\leftrightarrow}{D}_{2} q+\bar{q} \gamma_{3} \stackrel{\leftrightarrow}{D}_{3} q\right)$ \\
\hline$\left\langle x^{2}\right\rangle_{q}$ & $8_{1}^{-}$ & yes & 1 & $\bar{q} \gamma_{\{1} \stackrel{\leftrightarrow}{D}_{1} \stackrel{\leftrightarrow}{D}_{4\}} q-\frac{1}{2} \bar{q}\left(\gamma_{\{2} \stackrel{\leftrightarrow}{D}_{2} \stackrel{\leftrightarrow}{D}_{4\}}+\gamma_{\{3} \stackrel{\leftrightarrow}{D}_{3} \stackrel{\leftrightarrow}{D}_{4\}}\right) q$ \\
\hline$\left\langle x^{3}\right\rangle_{q}$ & $\mathbf{2}_{1}^{+}$ & no* & 1 & $\bar{q} \gamma_{\{1} \stackrel{\leftrightarrow}{D}_{1} \stackrel{\leftrightarrow}{D}_{4} \stackrel{\leftrightarrow}{D}_{4\}} q+\bar{q} \gamma_{\{2} \stackrel{\leftrightarrow}{D}_{2} \stackrel{\leftrightarrow}{D}_{3} \stackrel{\leftrightarrow}{D}_{3\}} q-(3 \leftrightarrow 4)$ \\
\hline$\langle 1\rangle_{\Delta q}$ & $4_{4}^{+}$ & no & 0 & $\bar{q} \gamma^{5} \gamma_{3} q$ \\
\hline$\langle x\rangle_{\Delta q}^{(a)}$ & $6_{3}^{-}$ & no & 1 & $\bar{q} \gamma^{5} \gamma_{\{1} \overleftrightarrow{D}_{3\}} q$ \\
\hline$\langle x\rangle_{\Delta q}^{(b)}$ & $6_{3}^{-}$ & no & 0 & $\bar{q} \gamma^{5} \gamma_{\{3} \overleftrightarrow{D}_{4\}} q$ \\
\hline$\left\langle x^{2}\right\rangle_{\Delta q}$ & $4_{2}^{+}$ & no & 1 & $\bar{q} \gamma^{5} \gamma_{\{1} \stackrel{\leftrightarrow}{D}_{3} \stackrel{\leftrightarrow}{D}_{4\}} q$ \\
\hline$\langle 1\rangle_{\delta q}$ & $\mathbf{6}_{1}^{+}$ & no & 0 & $\bar{q} \gamma^{5} \sigma_{34} q$ \\
\hline$\langle x\rangle_{\delta q}$ & $8_{1}^{-}$ & no & 1 & $\bar{q} \gamma^{5} \sigma_{3\{4} \stackrel{\leftrightarrow}{D}_{1\}} q$ \\
\hline$d_{1}$ & $\mathbf{6}_{1}^{+}$ & $\mathrm{no}^{* *}$ & 0 & $\bar{q} \gamma^{5} \gamma_{[3} \stackrel{\leftrightarrow}{D}_{4]} q$ \\
\hline$d_{2}$ & $8_{1}^{-}$ & $\mathrm{no}^{* *}$ & 1 & $\bar{q} \gamma^{5} \gamma_{[1} \stackrel{\leftrightarrow}{D}_{\{3]} \stackrel{\leftrightarrow}{D}_{4\}} q$ \\
\hline
\end{tabular}

TABLE II. Operators used to measure moments of quark distributions. Different lattice operators corresponding to the same continuum operator are denoted by superscripts $a$ and $b$. Subscripts of irreducible representations of $\mathrm{H}(4)$ distinguish different representations of the same dimensionality and superscripts denote charge conjugation C. In the operator mixing column, no* indicates a case in which mixing generically could exist but vanishes perturbatively for Wilson or overlap fermions and no** indicates perturbative mixing with lower dimension operators for Wilson fermions but no mixing for overlap fermions. The entry in column $\vec{P}$ denotes the number of spatial components of the nucleon momentum, $\vec{P}$, that must be chosen non-zero. Operators requiring one non-zero component have been written for $\vec{P}$ in the 1 - direction and $\vec{S}$ in the 3 -direction.

\begin{tabular}{lrrrrr}
\hline \hline observable & $\gamma$ & $B^{L A T T}$ & $B^{\overline{M S}}$ & $Z(\beta=6.0)$ & $Z(\beta=5.6)$ \\
\hline$\langle x\rangle_{q}^{(a)}$ & $8 / 3$ & -3.16486 & $-40 / 9$ & 0.9892 & 0.9884 \\
$\langle x\rangle_{q}^{(b)}$ & $8 / 3$ & -1.88259 & $-40 / 9$ & 0.9784 & 0.9768 \\
$\left\langle x^{2}\right\rangle_{q}$ & $25 / 6$ & -19.57184 & $-67 / 9$ & 1.1024 & 1.1097 \\
$\left\langle x^{3}\right\rangle_{q}$ & $157 / 30$ & -35.35192 & $-2216 / 225$ & 1.2153 & 1.2307 \\
$\langle 1\rangle_{\Delta q}$ & 0 & 15.79628 & 0 & 0.8666 & 0.8571 \\
$\langle x\rangle_{\Delta q}^{(a)}$ & $8 / 3$ & -4.09933 & $-40 / 9$ & 0.9971 & 0.9969 \\
$\langle x\rangle_{\Delta q}^{(b)}$ & $8 / 3$ & -4.09933 & $-40 / 9$ & 0.9971 & 0.9969 \\
$\left\langle x^{2}\right\rangle_{\Delta q}$ & $25 / 6$ & -19.56159 & $-67 / 9$ & 1.1023 & 1.1096 \\
$\langle 1\rangle_{\delta q}$ & 1 & 16.01808 & -1 & 0.8563 & 0.8461 \\
$\langle x\rangle_{\delta q}$ & 3 & -4.47754 & -5 & 0.9956 & 0.9953 \\
$d_{1}$ & 0 & 0.36500 & 0 & 0.9969 & 0.9967 \\
$d_{2}$ & $7 / 6$ & -15.67745 & $-35 / 18$ & 1.1159 & 1.1242 \\
\hline \hline
\end{tabular}

TABLE III. Renormalization constants 


\begin{tabular}{llllll}
\hline \hline & $\beta=6 / g_{0}^{2}$ & $\kappa_{\text {crit }}$ & $a_{N}(\mathrm{fm})$ & $a_{\rho}(\mathrm{fm})$ & Ref. \\
\hline LANL & 5.6 & 0.1585 & $0.086(14)$ & $0.076(9)$ & {$[74]$} \\
& 5.5 & 0.16145 & $0.116(7)$ & $0.104(5)$ & {$[74]$} \\
& 5.4 & 0.16450 & $0.164(13)$ & $0.136(9)$ & {$[74]$} \\
HEMCGC & 5.3 & 0.16794 & $0.124(4)$ & $0.108(2)$ & {$[75]$} \\
SCRI & 5.5 & 0.16116 & $0.109(4)$ & $0.090(3)$ & []$\left._{48}\right]$ \\
SESAM & 5.6 & 0.1585 & $0.091(16)$ & $0.076(10)$ & {$[16]$} \\
\hline \hline
\end{tabular}

TABLE IV. Parameters and lattice spacing for published dynamical Wilson fermion calculations. This table summarizes calculations on $16^{3} \times 32$ lattices and the resulting determinations of the lattice spacing from the masses of the nucleon, $a_{N}$, and from the $\rho$ meson, $a_{\rho}$.

\begin{tabular}{|c|c|c|c|c|}
\hline & $\kappa_{c}=0.1571$ & 0.1550 & 0.1540 & 0.1530 \\
\hline$\langle x\rangle_{u}^{(b)}$ & $0.454(29)$ & $0.458(17)$ & $0.464(10)$ & $0.465(7)$ \\
\hline$\langle x\rangle_{d}^{(b)}$ & $0.203(14)$ & $0.209(8)$ & $0.213(5)$ & $0.216(3)$ \\
\hline$\langle x\rangle_{u}^{(a)}$ & $0.308(125)$ & $0.375(64)$ & $0.402(47)$ & $0.437(41)$ \\
\hline$\langle x\rangle_{d}^{(a)}$ & $0.159(57)$ & $0.179(29)$ & $0.178(21)$ & $0.192(19)$ \\
\hline$\left\langle x^{2}\right\rangle_{u}$ & $0.119(61)$ & $0.135(32)$ & $0.129(23)$ & $0.142(20)$ \\
\hline$\left\langle x^{2}\right\rangle_{d}$ & $0.0286(324)$ & $0.0410(167)$ & $0.0464(118)$ & $0.0527(108)$ \\
\hline$\left\langle x^{3}\right\rangle_{u}$ & $0.0369(357)$ & $0.0441(192)$ & $0.0504(126)$ & $0.0532(107)$ \\
\hline$\left\langle x^{3}\right\rangle_{d}$ & $-0.00893(1842)$ & $0.000957(10126)$ & $0.00917(638)$ & $0.0132(53)$ \\
\hline$\langle 1\rangle_{\Delta u}$ & $0.888(80)$ & $0.915(46)$ & $0.926(28)$ & $0.939(20)$ \\
\hline$\langle 1\rangle_{\Delta d}$ & $-0.241(58)$ & $-0.244(38)$ & $-0.250(18)$ & $-0.251(12)$ \\
\hline$\overline{\langle x\rangle_{\Delta u}^{(b)}}$ & $0.215(25)$ & $0.228(14)$ & $0.238(9)$ & $0.243(7)$ \\
\hline$\langle x\rangle_{\Delta d}^{(b)}$ & $-0.0535(164)$ & $-0.0564(97)$ & $-0.0561(56)$ & $-0.0578(39)$ \\
\hline$\langle x\rangle_{\Delta u}^{(a)}$ & $0.141(123)$ & $0.199(65)$ & $0.220(45)$ & $0.251(37)$ \\
\hline$\langle x\rangle_{\Delta d}^{(a)}$ & $-0.00144(7536)$ & $-0.0200(407)$ & $-0.0338(283)$ & $-0.0415(215)$ \\
\hline$\left\langle x^{2}\right\rangle_{\Delta u}$ & $0.0269(428)$ & $0.0514(236)$ & $0.0639(152)$ & $0.0758(121)$ \\
\hline$\left\langle x^{2}\right\rangle_{\Delta d}$ & $0.00274(2530)$ & $-0.00555(1431)$ & $-0.0107(90)$ & $-0.0145(66)$ \\
\hline$\langle 1\rangle_{\delta u}$ & $1.014(82)$ & $1.035(49)$ & $1.045(28)$ & $1.055(20)$ \\
\hline$\langle 1\rangle_{\delta d}$ & $-0.199(46)$ & $-0.222(27)$ & $-0.241(16)$ & $-0.251(11)$ \\
\hline$\langle x\rangle_{\delta u}$ & $0.337(209)$ & $0.422(111)$ & $0.474(79)$ & $0.512(63)$ \\
\hline$\langle x\rangle_{\delta d}$ & $-0.0694(689)$ & $-0.0723(371)$ & $-0.0638(253)$ & $-0.0682(201)$ \\
\hline$d_{1}^{u}$ & $-1.354(75)$ & $-1.079(50)$ & $-0.914(24)$ & $-0.779(14)$ \\
\hline$d_{1}^{d}$ & $0.282(42)$ & $0.230(28)$ & $0.208(13)$ & $0.183(8)$ \\
\hline$d_{2}^{u}$ & $-0.233(86)$ & $-0.189(52)$ & $-0.172(29)$ & $-0.150(19)$ \\
\hline$d_{2}^{d}$ & $0.0396(311)$ & $0.0313(193)$ & $0.0230(104)$ & $0.0193(65)$ \\
\hline
\end{tabular}

TABLE V. Moments of quark distributions in quenched QCD calculated at three values of $\kappa$ and extrapolated linearly to $\kappa_{c}$. 


\begin{tabular}{|c|c|c|c|c|c|}
\hline & $\kappa_{c}=0.1585$ & $(0.1575)$ & 0.1570 & 0.1565 & 0.1560 \\
\hline$\overline{\langle x\rangle_{u}^{(b)}}$ & $0.459(29)$ & $0.503(14)$ & $0.470(13)$ & $0.449(9)$ & $0.461(5)$ \\
\hline$\langle x\rangle_{d}^{(b)}$ & $0.190(17)$ & $0.222(9)$ & $0.207(8)$ & $0.207(4)$ & $0.214(4)$ \\
\hline$\langle x\rangle_{u}^{(a)}$ & $0.462(174)$ & $0.412(93)$ & $0.411(69)$ & $0.439(70)$ & $0.394(45)$ \\
\hline$\langle x\rangle_{d}^{(a)}$ & $0.178(83)$ & $0.168(37)$ & $0.161(33)$ & $0.216(37)$ & $0.170(20)$ \\
\hline$\left\langle x^{2}\right\rangle_{u}$ & $0.176(63)$ & $0.131(34)$ & $0.134(24)$ & $0.131(24)$ & $0.110(18)$ \\
\hline$\left\langle x^{2}\right\rangle_{d}$ & $0.0314(303)$ & $0.0328(151)$ & $0.0414(109)$ & $0.0503(102)$ & $0.0496(101)$ \\
\hline$\left\langle x^{3}\right\rangle_{u}$ & $0.0685(392)$ & $0.0443(168)$ & $0.0521(152)$ & $0.0594(121)$ & $0.0466(113)$ \\
\hline$\left\langle x^{3}\right\rangle_{d}$ & $-0.00989(1529)$ & $0.0232(98)$ & $0.00789(549)$ & $0.0259(63)$ & $0.0225(50)$ \\
\hline$\langle 1\rangle_{\Delta u}$ & $0.860(69)$ & $0.741(37)$ & $0.880(28)$ & $0.975(24)$ & $0.936(16)$ \\
\hline$\langle 1\rangle_{\Delta d}$ & $-0.171(43)$ & $-0.214(25)$ & $-0.214(18)$ & $-0.248(15)$ & $-0.254(9)$ \\
\hline$\langle x\rangle_{\Delta u}^{(b)}$ & $0.242(22)$ & $0.241(15)$ & $0.237(9)$ & $0.237(7)$ & $0.235(6)$ \\
\hline$\langle x\rangle_{\Delta d}^{(b)}$ & $-0.0290(129)$ & $-0.0484(63)$ & $-0.0460(52)$ & $-0.0605(38)$ & $-0.0621(34)$ \\
\hline$\langle x\rangle_{\Delta u}^{(a)}$ & $0.254(111)$ & $0.205(83)$ & $0.196(43)$ & $0.217(41)$ & $0.171(31)$ \\
\hline$\langle x\rangle_{\Delta d}^{(a)}$ & $-0.0546(863)$ & $-0.0611(418)$ & $-0.0849(377)$ & $-0.0473(246)$ & $-0.0745(194)$ \\
\hline$\left\langle x^{2}\right\rangle_{\Delta u}$ & $0.116(42)$ & $0.0859(330)$ & $0.0673(161)$ & $0.0920(166)$ & $0.0483(114)$ \\
\hline$\left\langle x^{2}\right\rangle_{\Delta d}$ & $0.00142(2515)$ & $-0.0179(169)$ & $-0.0149(101)$ & $-0.0157(95)$ & $-0.0239(61)$ \\
\hline$\langle 1\rangle_{\delta u}$ & $0.963(59)$ & $0.919(40)$ & $1.023(26)$ & $1.062(18)$ & $1.075(13)$ \\
\hline$\langle 1\rangle_{\delta d}$ & $-0.202(36)$ & $-0.239(27)$ & $-0.238(15)$ & $-0.225(14)$ & $-0.250(7)$ \\
\hline$\langle x\rangle_{\delta u}$ & $0.477(196)$ & $0.424(109)$ & $0.418(79)$ & $0.465(75)$ & $0.405(48)$ \\
\hline$\langle x\rangle_{\delta d}$ & $-0.144(68)$ & $-0.0828(385)$ & $-0.115(28)$ & $-0.0565(248)$ & $-0.0739(167)$ \\
\hline$d_{1}^{u}$ & $-1.318(55)$ & $-1.036(44)$ & $-1.032(26)$ & $-0.957(15)$ & $-0.854(11)$ \\
\hline$d_{1}^{d}$ & $0.278(35)$ & $0.269(30)$ & $0.239(15)$ & $0.200(13)$ & $0.197(6)$ \\
\hline$d_{2}^{u}$ & $-0.228(81)$ & $-0.191(50)$ & $-0.179(34)$ & $-0.164(29)$ & -0. $147(17)$ \\
\hline$d_{2}^{d}$ & $0.0765(310)$ & $0.0392(181)$ & $0.0462(134)$ & $0.0172(112)$ & $0.0154(59)$ \\
\hline
\end{tabular}

TABLE VI. Moments of quark distributions in full QCD at $\beta=5.6$ using SESAM configurations. Lattice measurements are shown at four values of $\kappa$, but to avoid finite volume effects at the lightest quark mass $(\kappa=0.1575)$, only values at the three lowest $\kappa$ 's are extrapolated linearly to $\kappa_{c}$. 


\begin{tabular}{|c|c|c|c|c|}
\hline & $\kappa_{c}=0.16116$ & 0.1604 & 0.1600 & 0.1596 \\
\hline$\langle x\rangle_{u}^{(b)}$ & $0.416(41)$ & $0.459(25)$ & $0.451(16)$ & $0.480(9)$ \\
\hline$\langle x\rangle_{d}^{(b)}$ & $0.181(20)$ & $0.197(10)$ & $0.205(11)$ & $0.213(7)$ \\
\hline$\langle x\rangle_{u}^{(a)}$ & $0.289(132)$ & $0.372(68)$ & $0.420(64)$ & $0.462(47)$ \\
\hline$\langle x\rangle_{d}^{(a)}$ & $0.0430(523)$ & $0.119(26)$ & $0.182(29)$ & $0.209(20)$ \\
\hline$\left\langle x^{2}\right\rangle_{u}$ & $0.0191(429)$ & $0.0753(210)$ & $0.150(30)$ & $0.146(17)$ \\
\hline$\left\langle x^{2}\right\rangle_{d}$ & $0.0290(311)$ & $0.0435(171)$ & $0.0513(134)$ & $0.0590(94)$ \\
\hline$\left\langle x^{3}\right\rangle_{u}$ & $0.0341(365)$ & $0.0401(201)$ & $0.0500(152)$ & $0.0509(112)$ \\
\hline$\left\langle x^{3}\right\rangle_{d}$ & $0.0232(228)$ & $0.0229(138)$ & $0.00746(827)$ & $0.00975(583)$ \\
\hline$\langle 1\rangle_{\Delta u}$ & $0.635(94)$ & $0.713(52)$ & $0.838(40)$ & $0.852(27)$ \\
\hline$\langle 1\rangle_{\Delta d}$ & $-0.279(48)$ & $-0.263(25)$ & $-0.280(22)$ & $-0.259(16)$ \\
\hline$\overline{\langle x\rangle_{\Delta u}^{(b)}}$ & $0.180(34)$ & $0.204(20)$ & $0.209(13)$ & $0.223(9)$ \\
\hline$\langle x\rangle_{\Delta d}^{(b)}$ & $-0.0597(168)$ & $-0.0597(94)$ & $-0.0597(70)$ & $-0.0597(48)$ \\
\hline$\langle x\rangle_{\Delta u}^{(a)}$ & $0.278(123)$ & $0.217(66)$ & $0.255(57)$ & 0.192(39) \\
\hline$\langle x\rangle_{\Delta d}^{(a)}$ & $0.119(61)$ & $0.0419(316)$ & $-0.0147(365)$ & $-0.0459(201)$ \\
\hline$\left\langle x^{2}\right\rangle_{\Delta u}$ & $0.156(50)$ & $0.0987(265)$ & $0.117(28)$ & $0.0598(153)$ \\
\hline$\left\langle x^{2}\right\rangle_{\Delta d}$ & $0.00611(3014)$ & $-0.0214(159)$ & $0.0230(162)$ & $-0.0223(93)$ \\
\hline$\langle 1\rangle_{\delta u}$ & $0.675(75)$ & $0.868(42)$ & $0.980(31)$ & $1.080(22)$ \\
\hline$\langle 1\rangle_{\delta d}$ & $-0.214(55)$ & $-0.225(30)$ & $-0.277(25)$ & $-0.265(14)$ \\
\hline$\langle x\rangle_{\delta u}$ & $0.251(138)$ & $0.376(71)$ & $0.421(76)$ & $0.501(46)$ \\
\hline$\langle x\rangle_{\delta d}$ & $0.00912(7595)$ & $0.0204(412)$ & $-0.106(31)$ & $-0.0527(253)$ \\
\hline$d_{1}^{u}$ & $-1.261(89)$ & $-1.197(56)$ & $-1.068(32)$ & $-1.046(21)$ \\
\hline$d_{1}^{d}$ & $0.374(67)$ & $0.302(41)$ & $0.301(27)$ & $0.256(13)$ \\
\hline$d_{2}^{u}$ & $-0.264(77)$ & $-0.236(42)$ & $-0.203(40)$ & $-0.196(21)$ \\
\hline$d_{2}^{d}$ & $0.0191(402)$ & $0.00394(2320)$ & $0.0501(172)$ & $0.0266(100)$ \\
\hline
\end{tabular}

TABLE VII. Moments of quark distributions in full QCD at $\beta=5.5$ using SCRI configurations extrapolated linearly to $\kappa_{c}$. 


\begin{tabular}{|l|c|cc|}
\hline \hline & $\kappa_{c}=0.1266$ & 0.1235 & 0.1246 \\
\hline \hline$\langle x\rangle_{u}^{(b)}$ & $0.565(61)$ & $0.581(8)$ & $0.575(21)$ \\
$\langle x\rangle_{d}^{(b)}$ & $0.238(29)$ & $0.265(5)$ & $0.255(10)$ \\
\hline$\langle x\rangle_{u}^{(a)}$ & $0.314(189)$ & $0.555(74)$ & $0.468(49)$ \\
$\langle x\rangle_{d}^{(a)}$ & $0.119(89)$ & $0.247(33)$ & $0.201(24)$ \\
\hline$\left\langle x^{2}\right\rangle_{u}$ & $0.146(78)$ & $0.169(29)$ & $0.161(21)$ \\
$\left\langle x^{2}\right\rangle_{d}$ & $0.0316(334)$ & $0.0716(123)$ & $0.0572(91)$ \\
\hline$\left\langle x^{3}\right\rangle_{u}$ & $0.0517(337)$ & $0.0761(130)$ & $0.0673(88)$ \\
$\left\langle x^{3}\right\rangle_{d}$ & $0.00911(1390)$ & $0.0309(55)$ & $0.0231(35)$ \\
\hline$\langle 1\rangle_{\Delta u}$ & $0.585(82)$ & $0.818(17)$ & $0.734(28)$ \\
$\langle 1\rangle_{\Delta d}$ & $-0.298(46)$ & $-0.210(8)$ & $-0.242(16)$ \\
\hline$\langle x\rangle_{\Delta u}^{(b)}$ & $0.118(46)$ & $0.114(17)$ & $0.115(13)$ \\
$\langle x\rangle_{\Delta d}^{(b)}$ & $-0.0120(242)$ & $-0.0230(57)$ & $-0.0191(79)$ \\
\hline$\langle x\rangle_{\Delta u}^{(a)}$ & $0.279(30)$ & $0.316(8)$ & $0.303(9)$ \\
$\langle x\rangle_{\Delta d}^{(a)}$ & $-0.0763(188)$ & $-0.0650(46)$ & $-0.0691(61)$ \\
\hline$\left\langle x^{2}\right\rangle_{\Delta u}$ & $0.236(117)$ & $0.274(40)$ & $0.261(34)$ \\
$\left\langle x^{2}\right\rangle_{\Delta d}$ & $-0.0650(782)$ & $-0.0534(200)$ & $-0.0576(251)$ \\
\hline$\langle 1\rangle_{\delta u}$ & $0.768(65)$ & $0.951(13)$ & $0.885(22)$ \\
$\langle 1\rangle_{\delta d}$ & $-0.289(49)$ & $-0.234(7)$ & $-0.254(17)$ \\
\hline$\langle x\rangle_{\delta u}$ & $0.451(265)$ & $0.702(96)$ & $0.611(73)$ \\
$\langle x\rangle_{\delta d}$ & $0.0153(936)$ & $-0.137(27)$ & $-0.0820(288)$ \\
\hline$d_{1}^{u}$ & $-0.101(7)$ & $0.155(2)$ & $0.0628(22)$ \\
$d_{1}^{d}$ & $0.00915(595)$ & $-0.0398(12)$ & $0.00808(409)$ \\
\hline$d_{2}^{u}$ & $-0.0462(220)$ & $-0.0164(34)$ & $0.0619(95)$ \\
$d_{2}^{d}$ & $0.00673(1288)$ & $0.020)$ \\
\hline \hline
\end{tabular}

TABLE VIII. Moments of quark distributions calculated with cooled configurations, as described in the text, to eliminate most of the gluon degrees of freedom except instantons. Lattice measurements at $\kappa=0.1235$ and 0.1246 are extrapolated linearly to $\kappa_{c}$. 


\begin{tabular}{|c|c|c|c|c|c|c|}
\hline $\begin{array}{l}\text { Connected } \\
\text { M. E. }\end{array}$ & QCDSF & $\begin{array}{r}\text { QCDSF } \\
(a=0)\end{array}$ & Wuppertal & Quenched & $\begin{array}{r}\text { Full QCD } \\
(3 \mathrm{pts})\end{array}$ & $\begin{array}{l}\text { Phenomenology } \\
\qquad(q \pm \bar{q})\end{array}$ \\
\hline$\overline{\langle x\rangle_{u}}$ & $0.452(26)$ & & & $0.454(29)$ & $0.459(29)$ & \\
\hline$\langle x\rangle_{d}$ & $0.189(12)$ & & & $0.203(14)$ & $0.190(17)$ & \\
\hline$\langle x\rangle_{u-d}$ & $0.263(17)$ & & & $0.251(18)$ & $0.269(23)$ & $0.154(3)$ \\
\hline$\left\langle x^{2}\right\rangle_{u}$ & $0.104(20)$ & & & $0.119(61)$ & $0.176(63)$ & \\
\hline$\left\langle x^{2}\right\rangle_{d}$ & $0.037(10)$ & & & $0.029(32)$ & $0.031(30)$ & \\
\hline$\left\langle x^{2}\right\rangle_{u-d}$ & $0.067(22)$ & & & $0.090(68)$ & $0.145(69)$ & $0.055(1)$ \\
\hline$\left\langle x^{3}\right\rangle_{u}$ & $0.022(11)$ & & & $0.037(36)$ & $0.069(39)$ & \\
\hline$\left\langle x^{3}\right\rangle_{d}$ & $-0.001(7)$ & & & $0.009(18)$ & $-0.010(15)$ & \\
\hline$\left\langle x^{3}\right\rangle_{u-d}$ & $0.023(13)$ & & & $0.028(49)$ & $0.078(41)$ & $0.023(1)$ \\
\hline$\langle 1\rangle_{\Delta u}$ & $0.830(70)$ & $0.889(29)$ & $0.816(20)$ & $0.888(80)$ & $0.860(69)$ & \\
\hline$\langle 1\rangle_{\Delta d}$ & $-0.244(22)$ & $-0.236(27)$ & $-0.237(9)$ & $-0.241(58)$ & $-0.171(43)$ & \\
\hline$\langle 1\rangle_{\Delta u-\Delta d}$ & 1.074(90) & $1.14(3)$ & $1.053(27)$ & $1.129(98)$ & $1.031(81)$ & $1.248(2)$ \\
\hline$\langle x\rangle_{\Delta u}$ & $0.198(8)$ & & & $0.215(25)$ & $0.242(22)$ & \\
\hline$\langle x\rangle_{\Delta d}$ & $-0.048(3)$ & & & $-0.054(16)$ & $-0.029(13)$ & \\
\hline$\langle x\rangle_{\Delta u-\Delta d}$ & $0.246(9)$ & & & $0.269(29)$ & $0.271(25)$ & $0.196(9)$ \\
\hline$\left\langle x^{2}\right\rangle_{\Delta u}$ & $0.04(2)$ & & & $0.027(60)$ & $0.116(42)$ & \\
\hline$\left\langle x^{2}\right\rangle_{\Delta d}$ & $-0.012(6)$ & & & $-0.003(25)$ & $0.001(25)$ & \\
\hline$\left\langle x^{2}\right\rangle_{\Delta u-\Delta d}$ & $0.05(2)$ & & & $0.030(65)$ & $0.115(49)$ & $0.061(6)$ \\
\hline$\delta u_{c}$ & $0.93(3)$ & $0.980(30)$ & & $1.01(8)$ & $0.963(59)$ & \\
\hline$\delta d_{c}$ & $-0.20(2)$ & $-0.234(17)$ & & $-0.20(5)$ & $-0.202(36)$ & \\
\hline$d_{2}^{u}$ & $-0.206(18)$ & & & $-0.233(86)$ & $-0.228(81)$ & \\
\hline$d_{2}^{d}$ & $-0.035(6)$ & & & $0.040(31)$ & $0.077(31)$ & \\
\hline
\end{tabular}

TABLE IX. Comparison of linear extrapolations of full QCD and quenched results with other lattice calculations and phenomenology at $4 \mathrm{GeV}^{2}$ in the $\overline{M S}$ scheme. The first column shows quenched results by the QCDSF collaboration at $\beta=6.0$ [10,52 55 and the second column shows extrapolation of several moments to the continuum limit 15]. The third column shows full QCD results calculated using a different method with the same SESAM configurations we have used [14. The quenched and full QCD results calculated in this work are shown in the fourth and fifth columns. Flavor non-singlet moments $\left\langle x^{n}\right\rangle$ of $q(x)+(-1)^{n+1} \bar{q}(x), \Delta q(x)+(-1)^{n} \Delta \bar{q}(x)$, and $\delta q(x)+(-1)^{n+1} \delta \bar{q}(x)$ are tabulated in the final column. Phenomenological unpolarized distributions are calculated from refs. [1] 3] and polarized distributions are calculated from refs. [4,5] with error estimates as described in the text. 


\section{FIGURES}
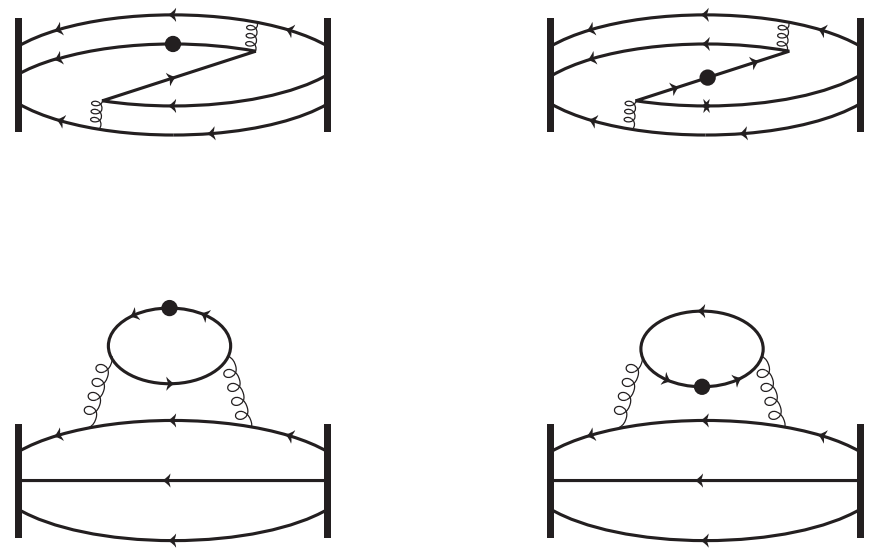

FIG. 1. Connected (upper row) and disconnected (lower row) diagrams contributing to hadron matrix elements. The left column shows typical contributions of quarks and the right column shows contributions of antiquarks.

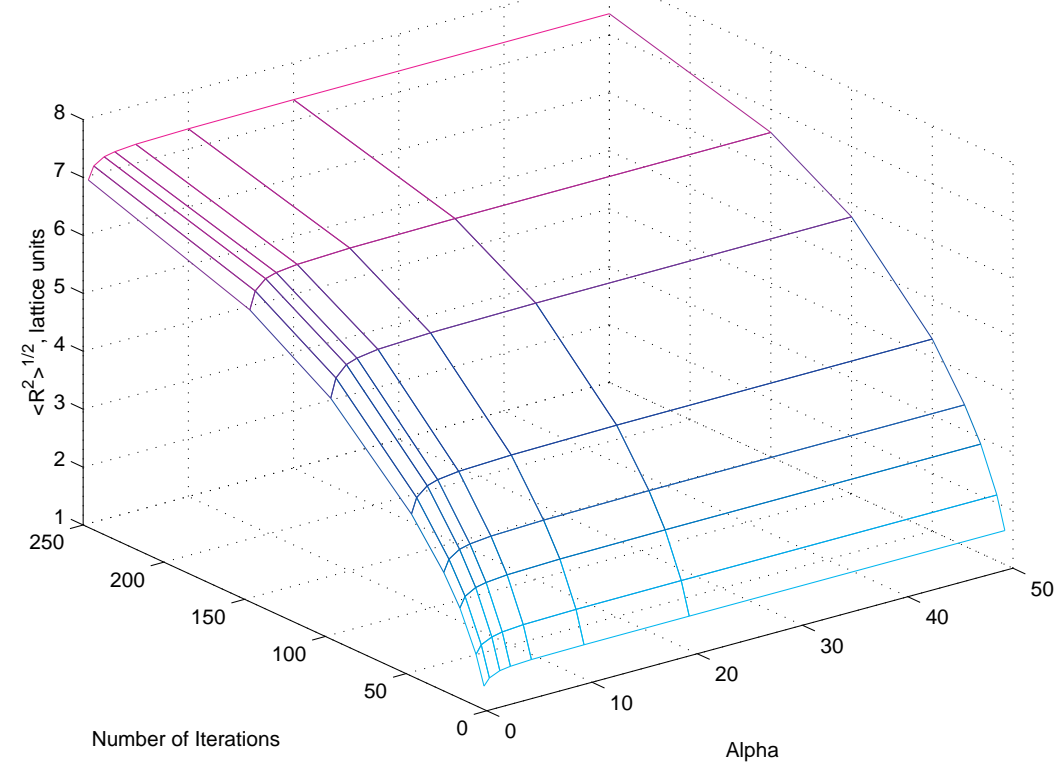

FIG. 2. The rms radius of a gauge invariant smeared quark source as a function of the coefficient $\alpha$ and number of smearing steps $N$ defined in Eq. 13 . 

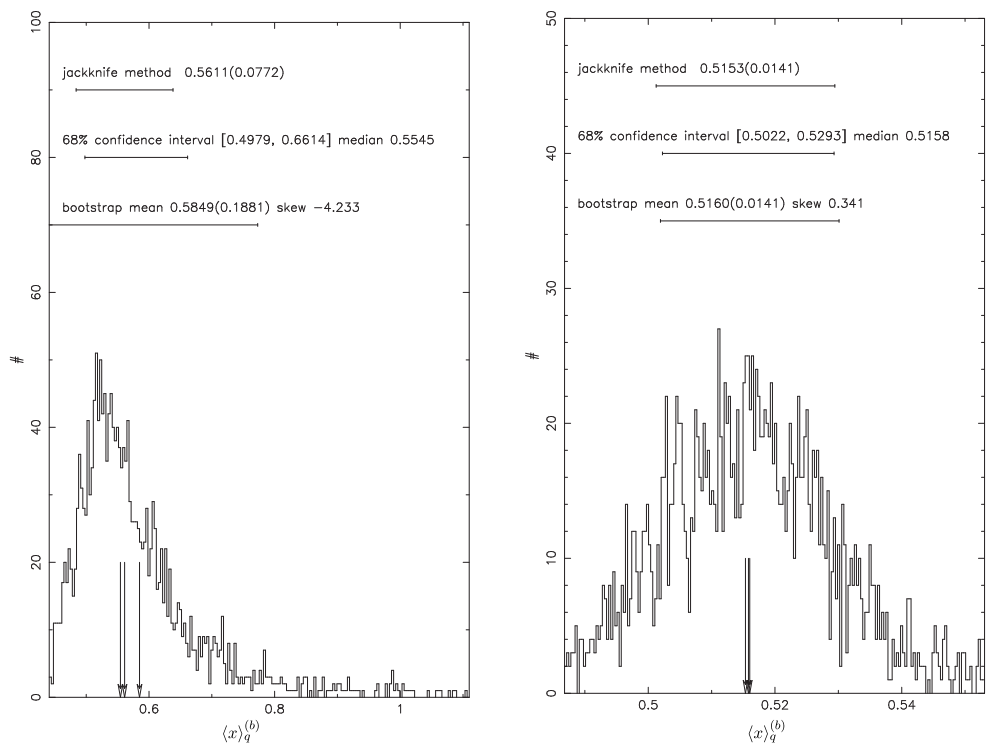

FIG. 3. Bootstrap distributions of $\langle x\rangle_{q}^{(b)}$ for ensembles of 25 (left plot) and 204 (right plot) SESAM configurations at $\kappa=0.1575$

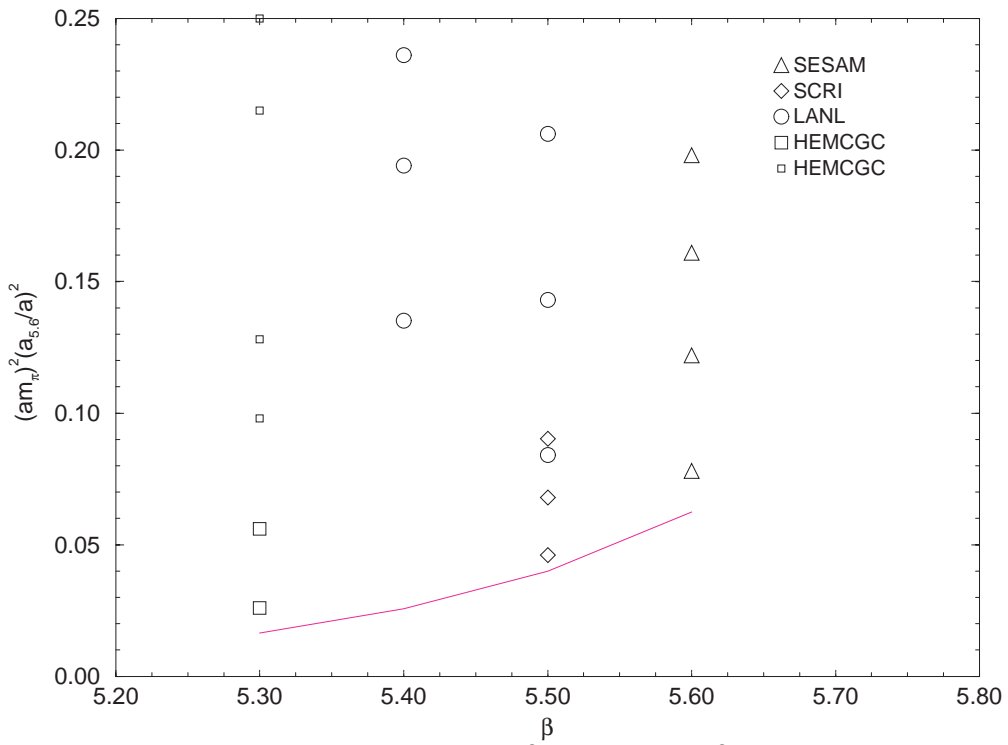

FIG. 4. The scaled pion mass squared, $\left(a m_{\pi}\right)^{2} \times\left(a_{5.6} / a\right)^{2}$, for the dynamical Wilson fermion calculations tabulated in Table IV. The dotted line shows the mass at which the pion Compton wavelength equals one-fourth of the spatial dimension. 


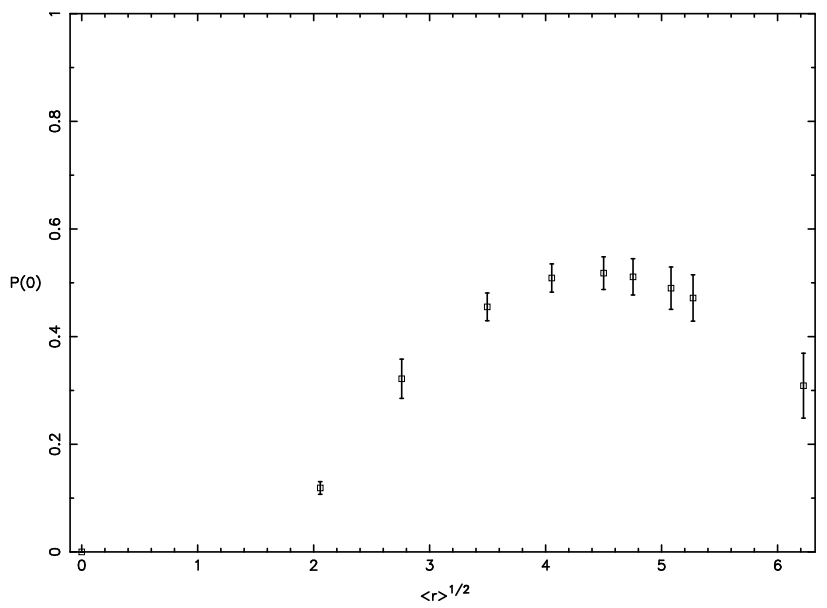

FIG. 5. Overlap between a smeared source and the proton ground state as a function of the source rms radius. The overlap for zero smearing is $6 \times 10^{-5}$.
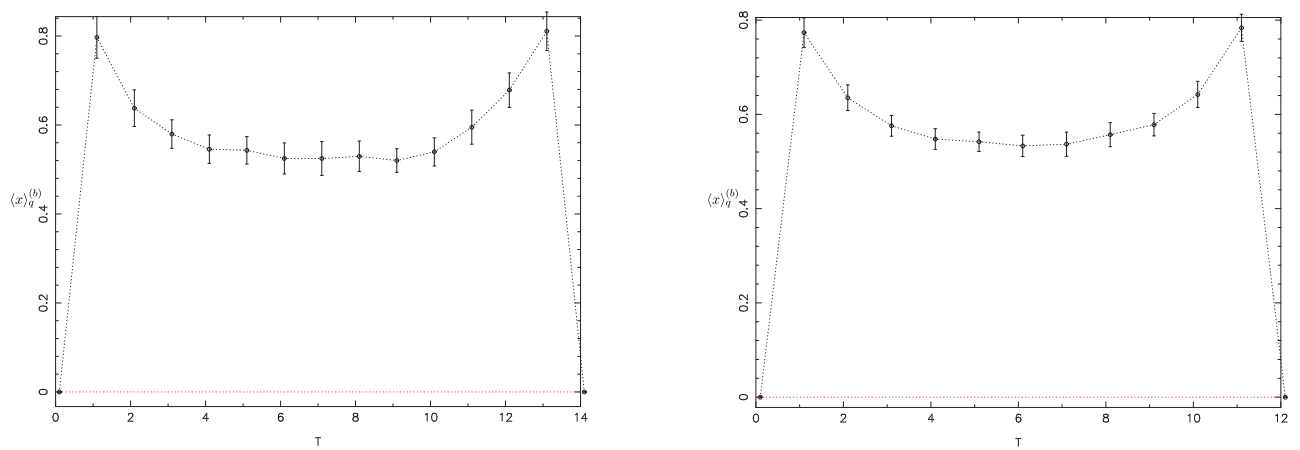

FIG. 6. Plateaus for $\langle x\rangle_{q}^{(b)}$ calculated with point sources separated by 14 (left) and 12 (right) time steps using 100 configurations at $\beta=5.6$ and $\kappa=.1575$. 


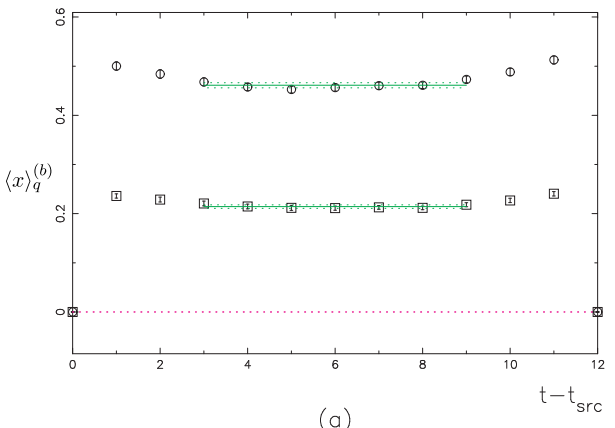

(a)

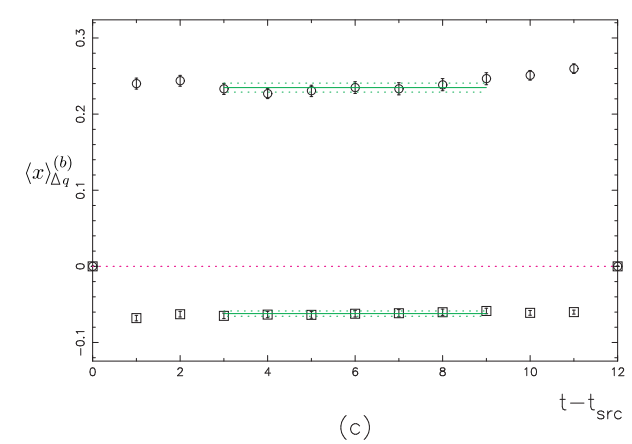

(c)

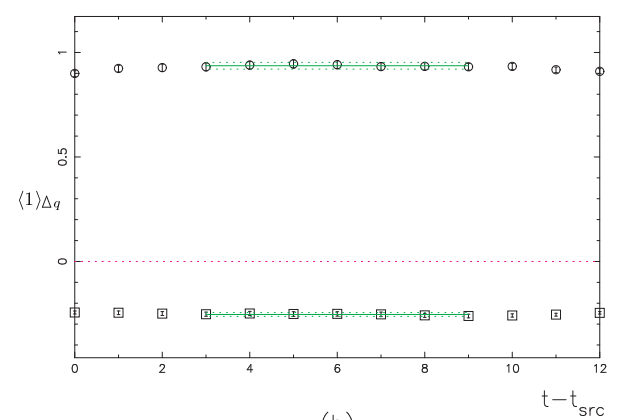

(b)

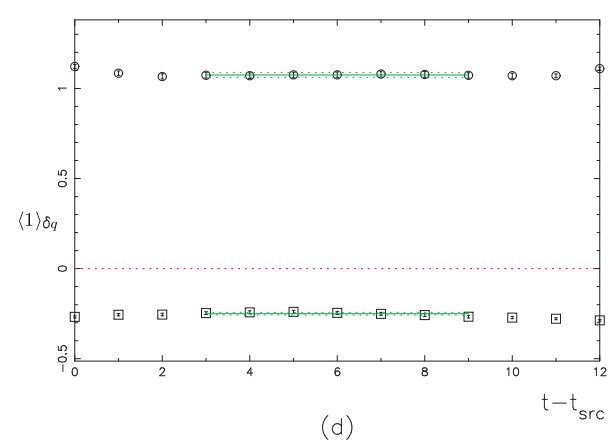

(d)

FIG. 7. Plateaus obtained using optimally smeared sources with $\alpha=3$ and $N=40$ separated by 12 time steps on SESAM configurations with $\beta=5.6$ and $\kappa=0.1560$. Measurements as a function of Euclidean time of the operators $\langle x\rangle_{q}^{(b)},\langle 1\rangle_{\Delta q},\langle x\rangle_{\Delta q}^{(b)}$, and $\langle 1\rangle_{\delta q}$ are shown in panels a, $\mathrm{b}$, c, and d respectively. Circles and squares denote matrix elements for up and down quarks respectively, the error bars are smaller than the symbols and shown within them, and the solid lines denote fits within the plateau region. 

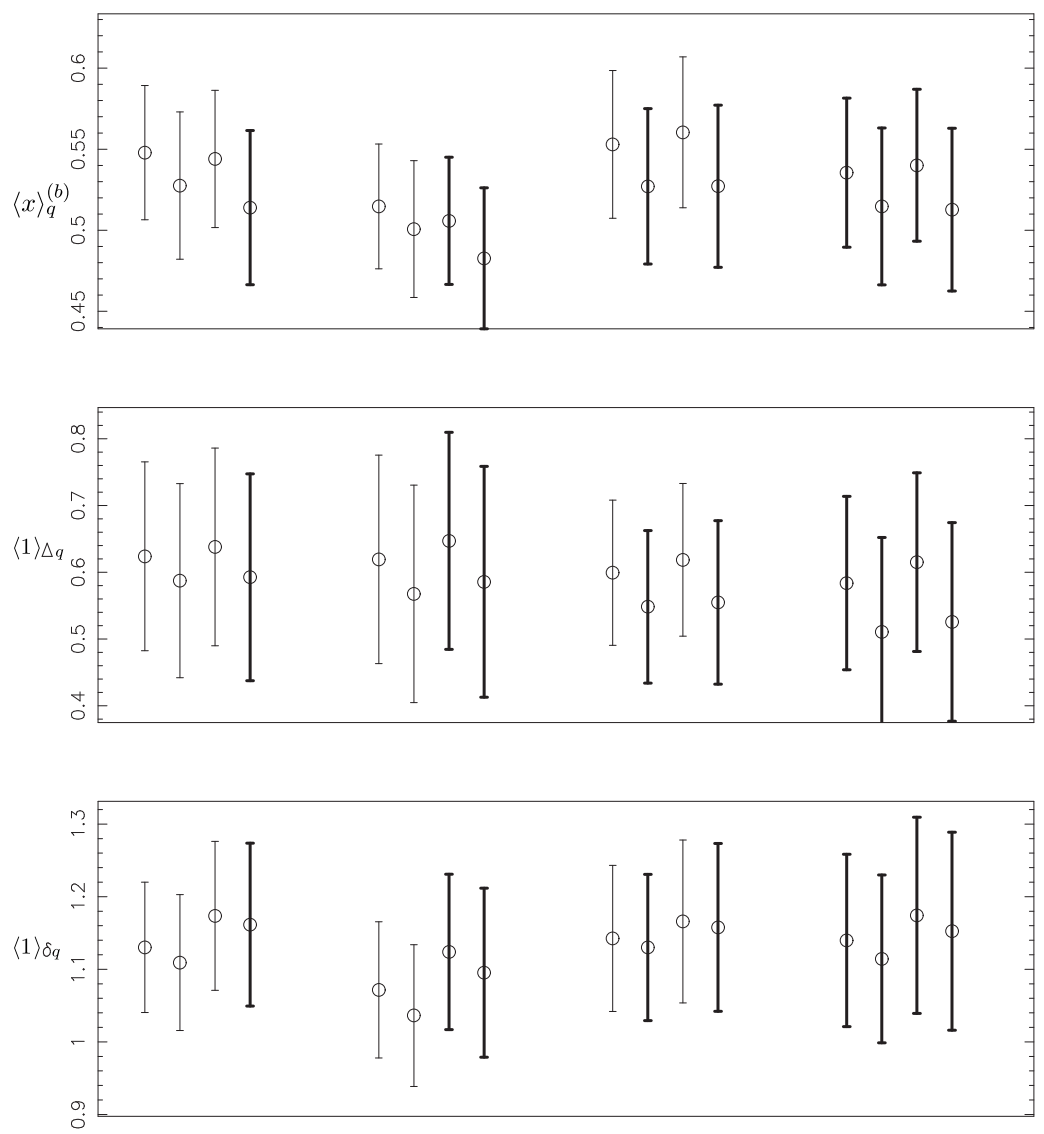

FIG. 8. Comparison of operator measurements using combinations of point and smeared sources and sinks. From left to right, the four clusters, each containing four error bars, correspond to point-point, point-smeared, smeared-point and smeared-smeared source-sink combinations respectively. Within each cluster, from left to right, the four error bars correspond to windows in which the number of lattice points omitted from the window at the source and sink are $(3,3),(3,5),(5,3)$, and $(5,5)$ respectively. All smeared sources and sinks have $N=20$ and $\alpha=3$. From top to bottom, the panels show measurements of the operators $\langle x\rangle_{q}^{(b)},\langle 1\rangle_{\Delta q}$, and $\langle 1\rangle_{\delta q}$. 

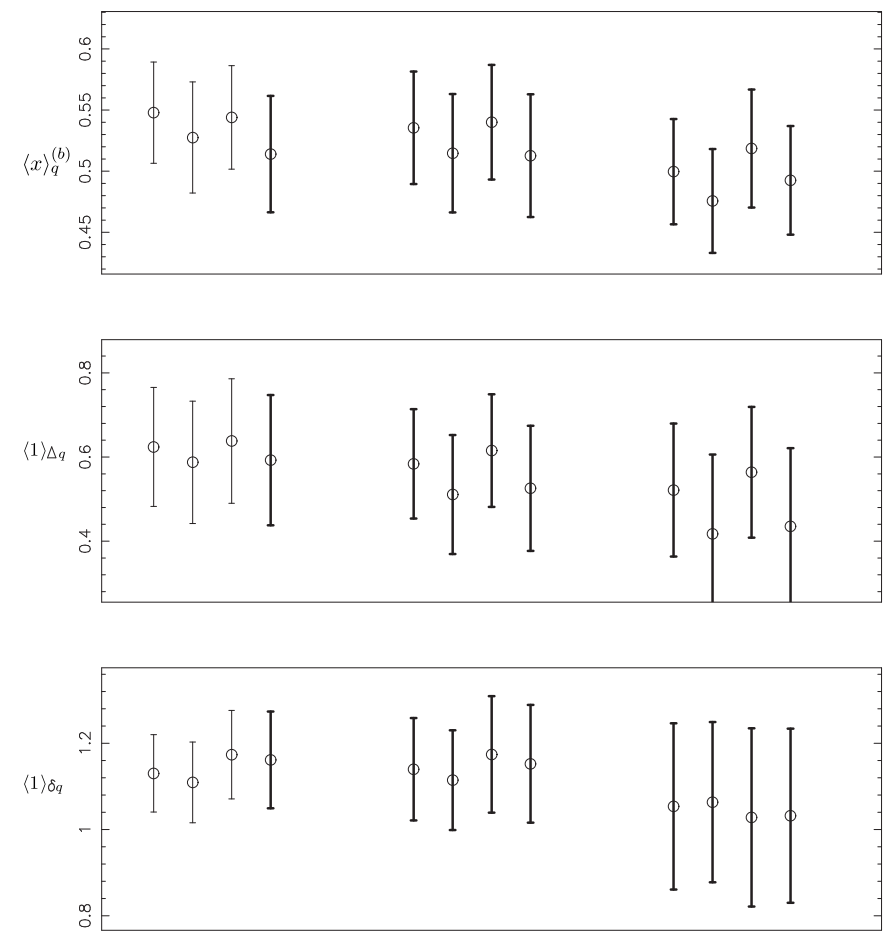

FIG. 9. Comparison of operator measurements using smeared sources of different sizes. From left to right, the three clusters, each containing four error bars, correspond to $N=0, N=20$, and $N=100$. The windows within each cluster and the operators are the same as in Fig 8 . 

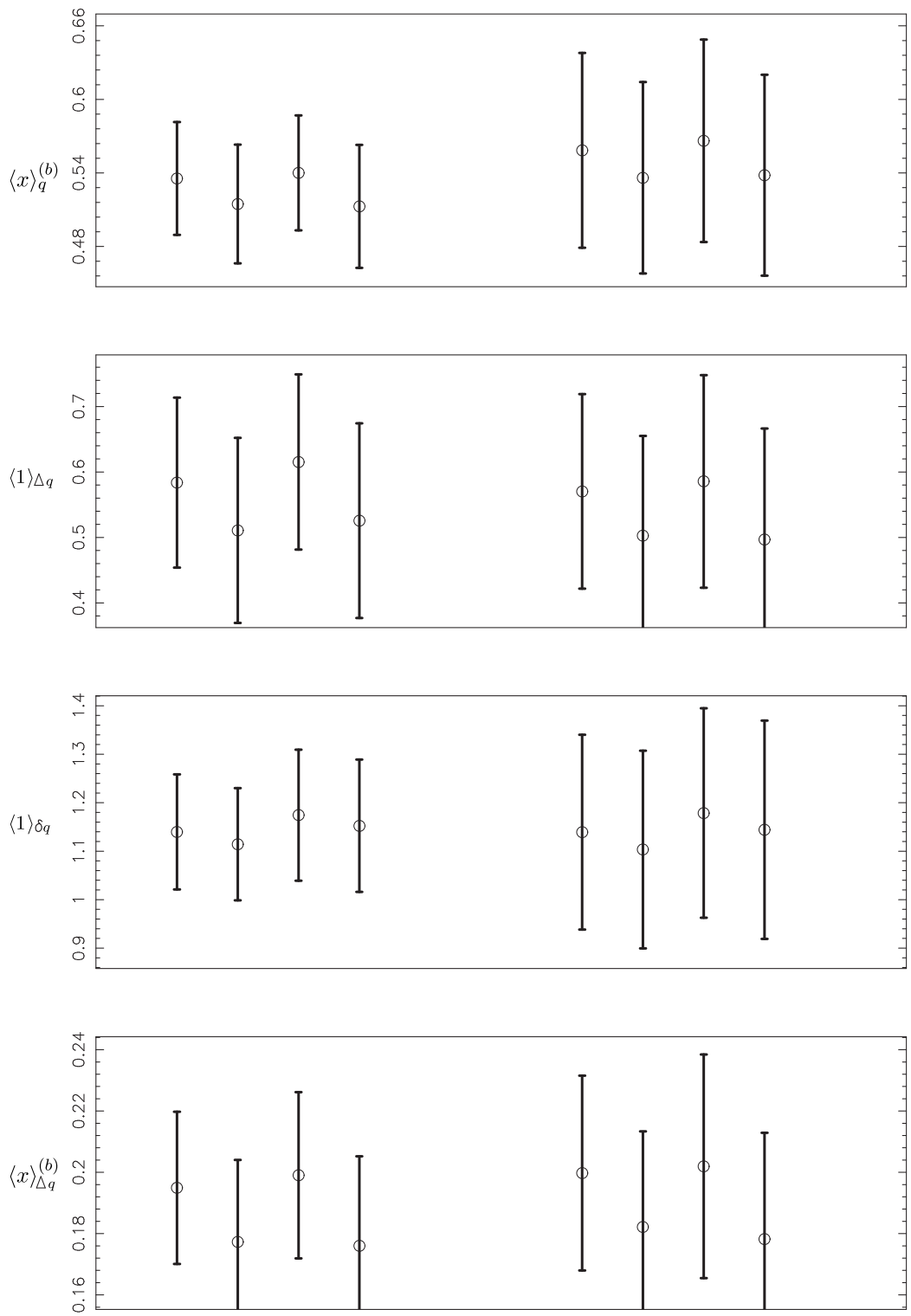

FIG. 10. Comparison of operator measurements using Dirichlet boundary conditions (left cluster) and periodic boundary conditions (right cluster). The windows within each cluster and the operators for the top three panels are the same as in Fig 8, and the bottom panel corresponds to the operator $\langle x\rangle_{\Delta q}$. 

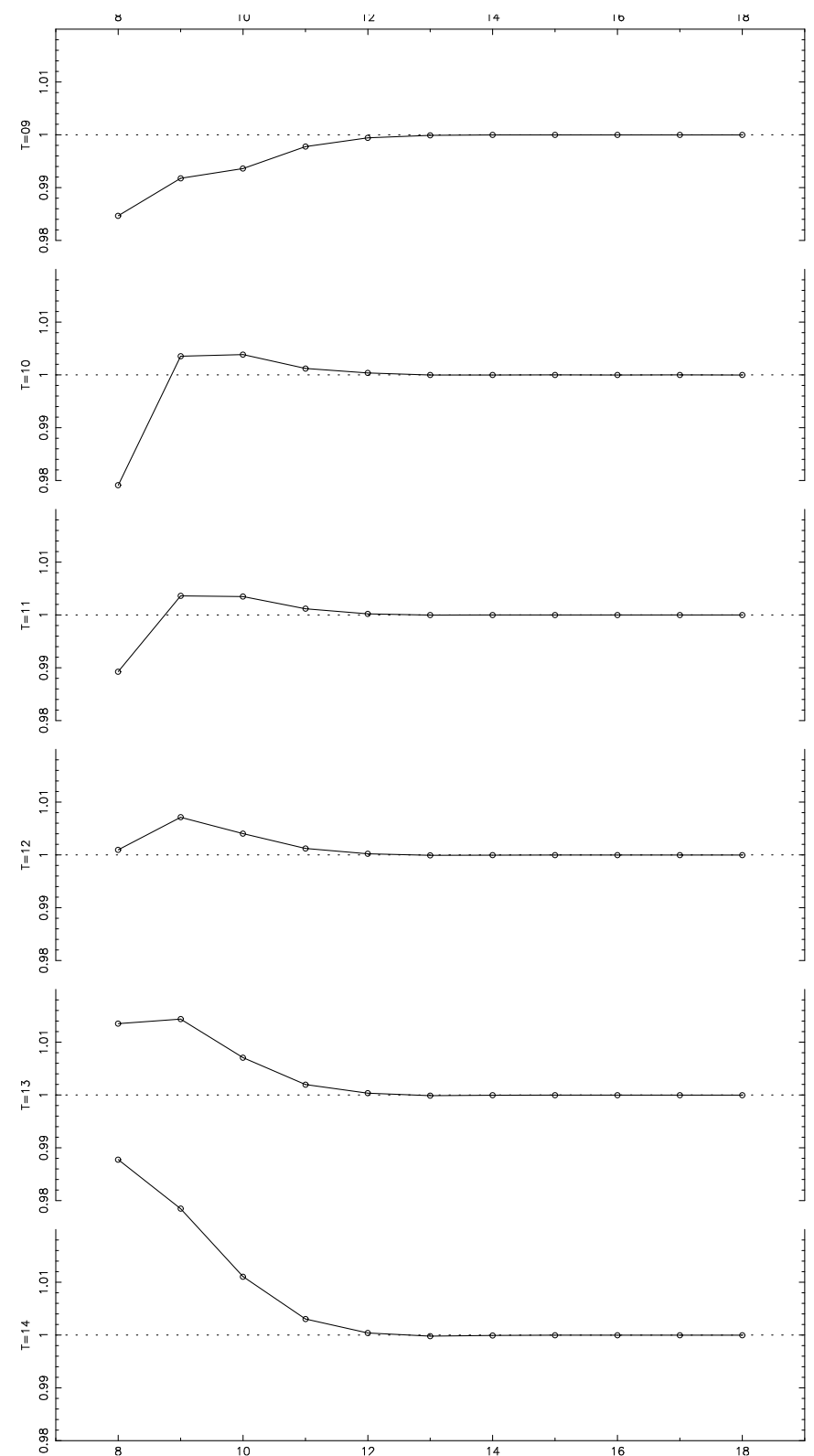

FIG. 11. Discrepancy between the axial charge, $\langle 1\rangle_{\Delta q}$, calculated in single precision (solid curve) and double precision (dotted curve) as a function of $\left(-\log _{10} r_{\min }^{2}\right)$ where $r_{\min }^{2}$ is the conjugate gradient stopping residue. From the top panel to the bottom panel, the source-sink separation increases from 9 to 14 lattice spacings. 


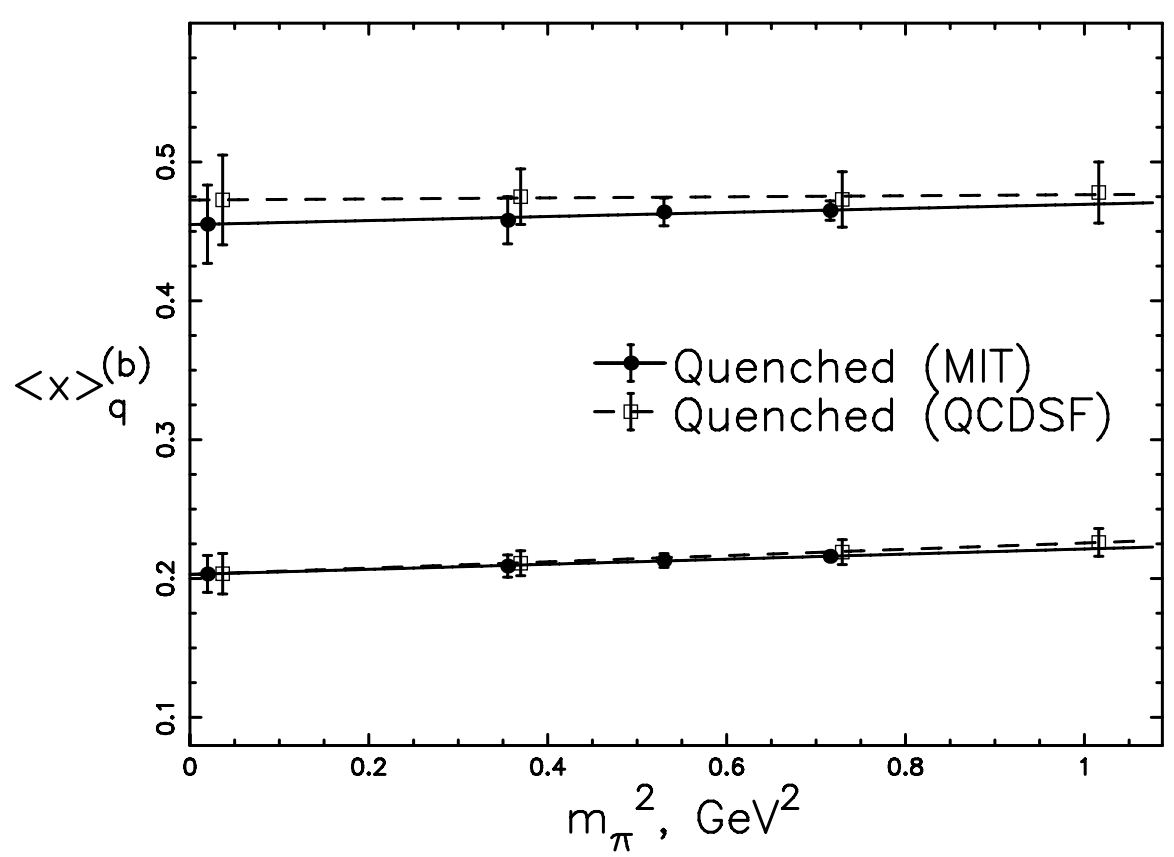

FIG. 12. Linear extrapolation of quenched calculations of the momentum fraction $\langle x\rangle_{q}^{(b)}$. The solid squares denote the results of the present calculation, and for comparison, QCDSF results are shown by the open points. Here and in subsequent figures, the upper and lower curves correspond to up and down quarks respectively.

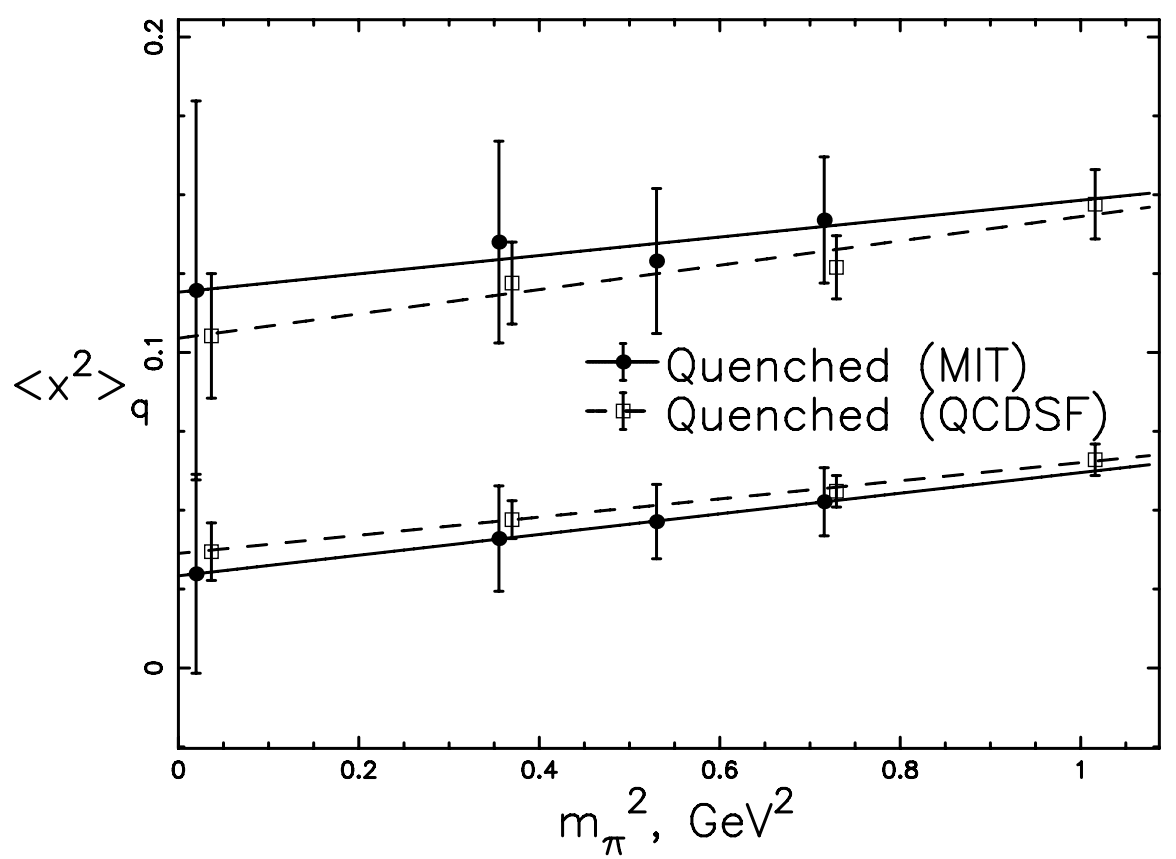

FIG. 13. Linear extrapolation of quenched calculations of $\left\langle x^{2}\right\rangle_{q}$, where the symbols are as in Fig. 12. 


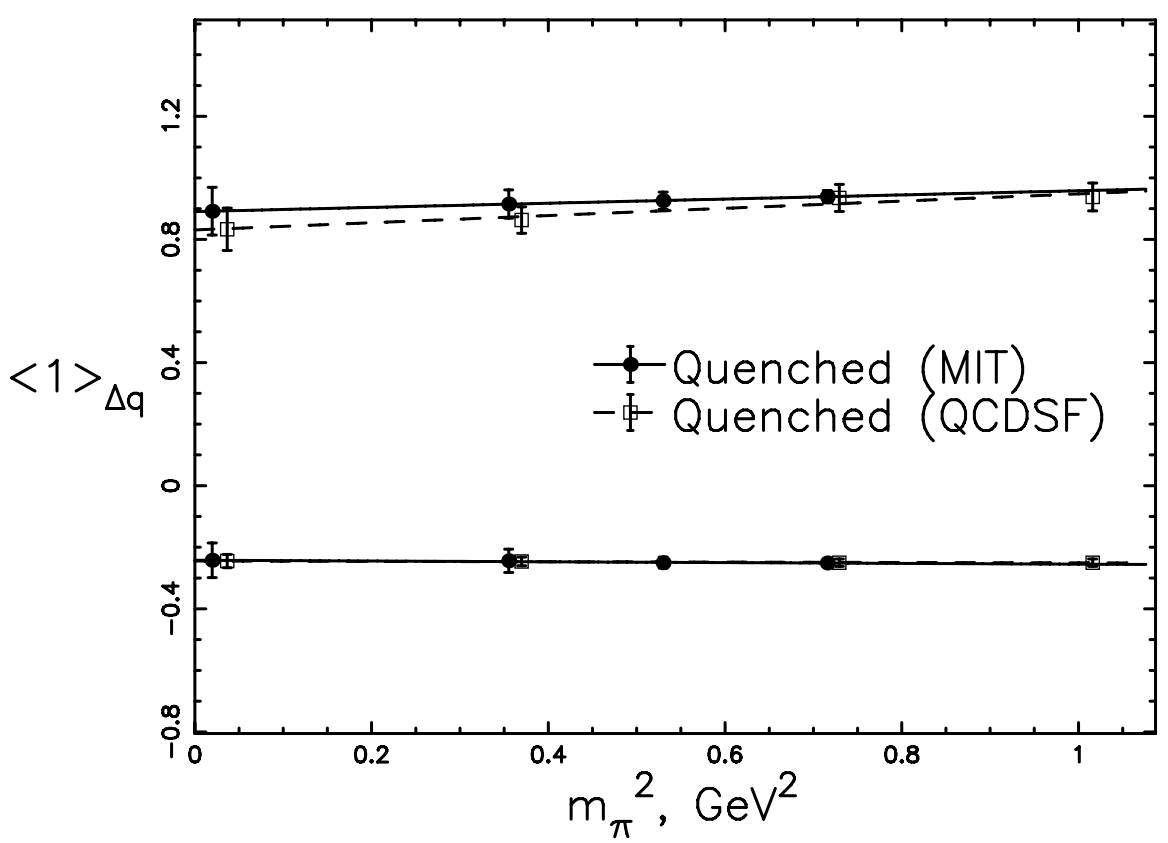

FIG. 14. Linear extrapolation of quenched calculations of the axial charge, $\langle 1\rangle_{\Delta q}$, where the symbols are as in Fig. 12 .

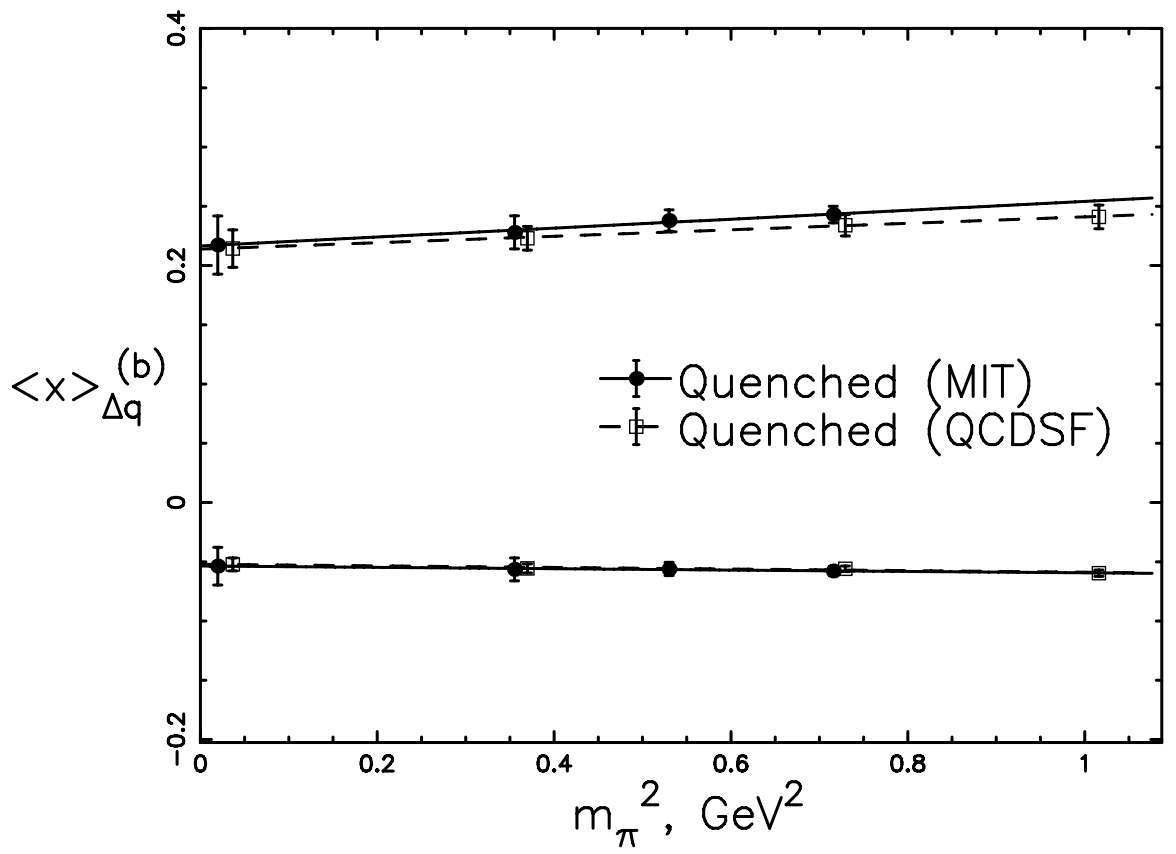

FIG. 15. Linear extrapolation of quenched calculations of the moment $\langle x\rangle_{\Delta q}^{(b)}$, where the symbols are as in Fig. 12. 


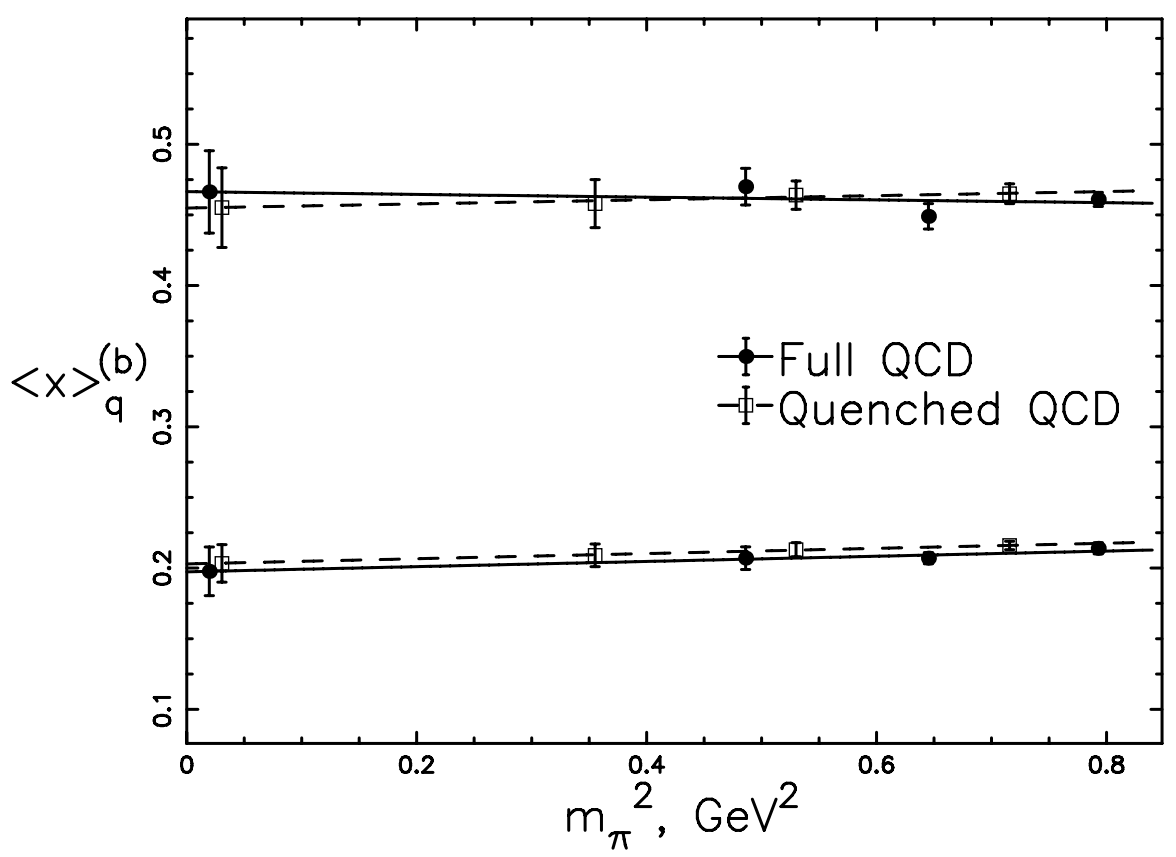

FIG. 16. Comparison of linear extrapolations of quenched and full QCD calculations of the momentum fraction, $\langle x\rangle_{q}$. The open symbols denote quenched calculations at $\beta=6.0$ and the closed symbols denote full QCD calculations at $\beta=5.6$. The upper and lower curves correspond to up and down quarks respectively.

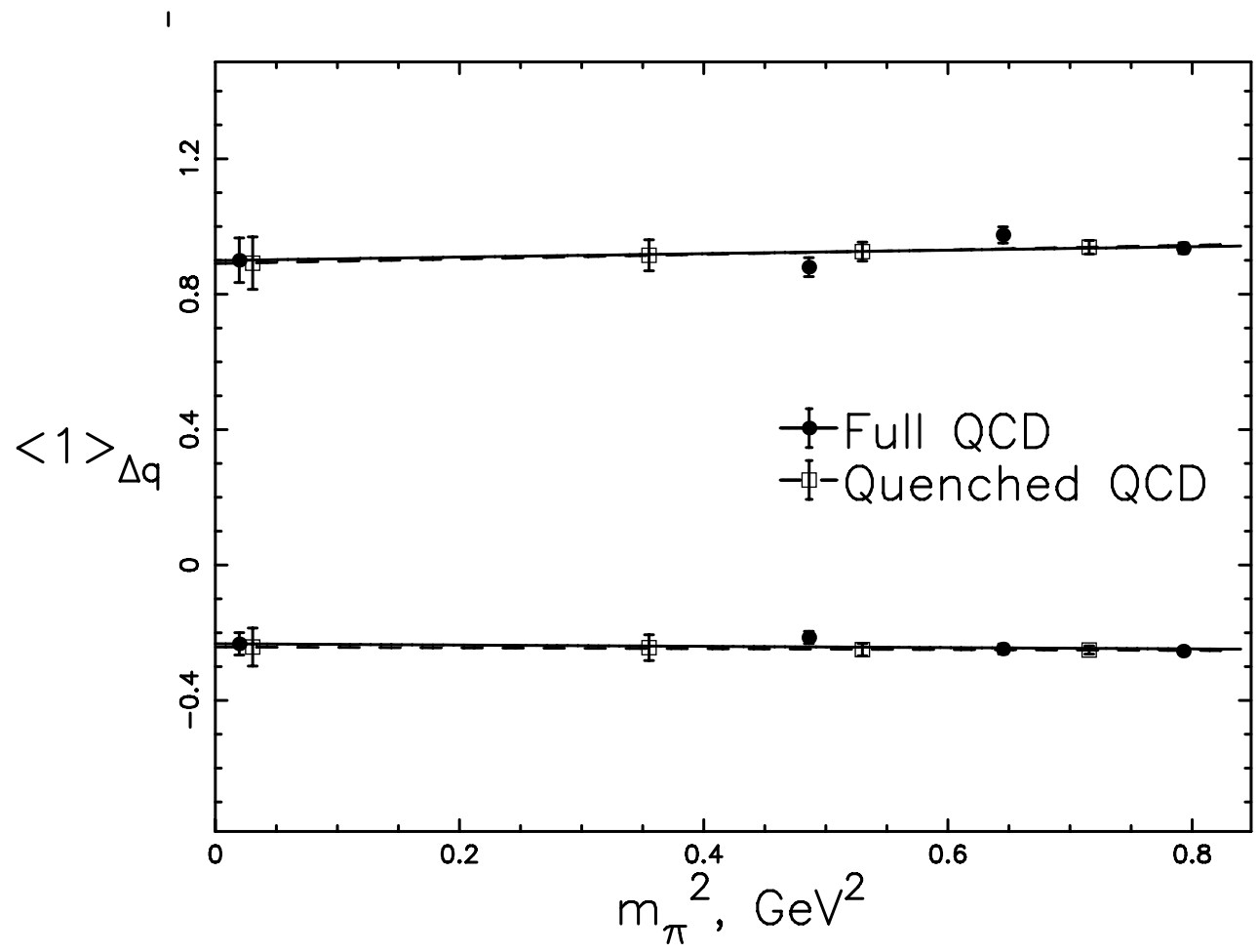

FIG. 17. Comparison of linear extrapolations of quenched and full QCD calculations of the axial charge, $\langle 1\rangle_{\Delta q}$, where the symbols are as in Fig. 16. 


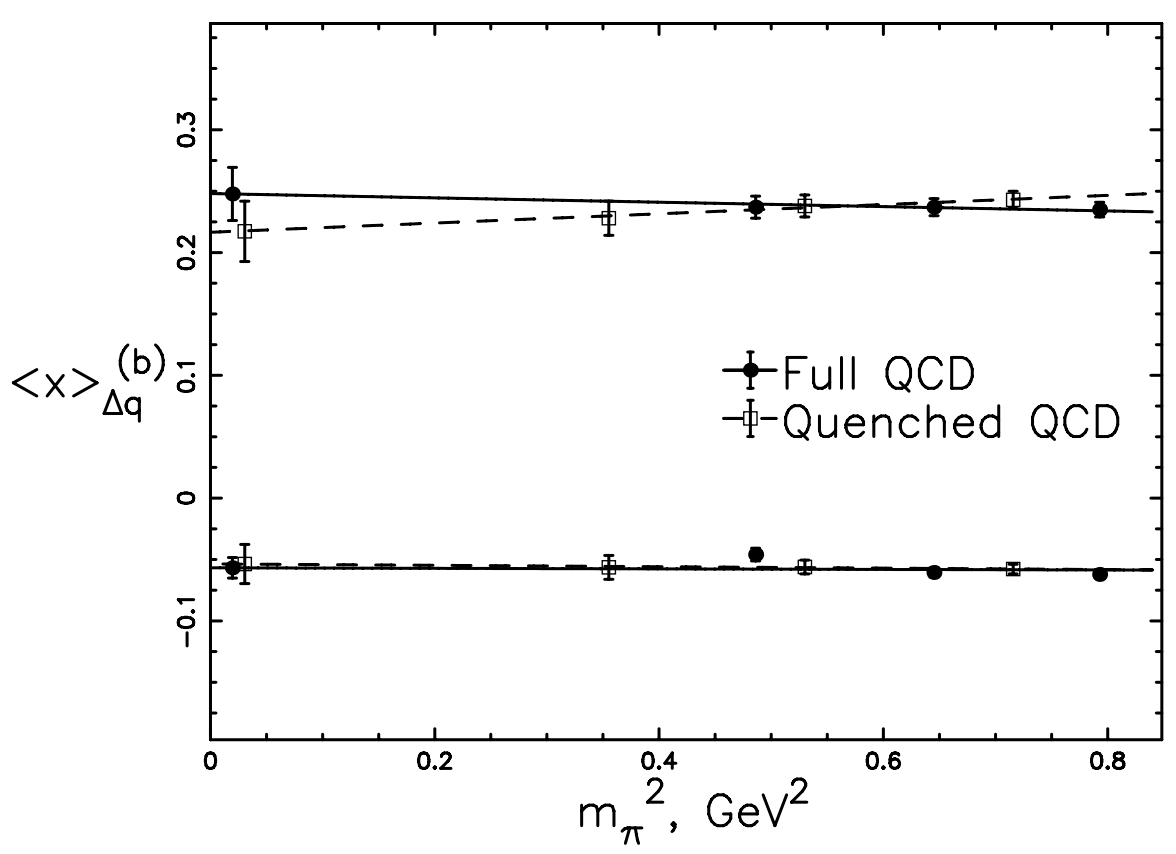

FIG. 18. Comparison of linear extrapolations of quenched and full QCD calculations of the first moment of the quark spin distribution, $\langle x\rangle_{\Delta q}^{(b)}$, where the symbols are as in Fig. 16 .

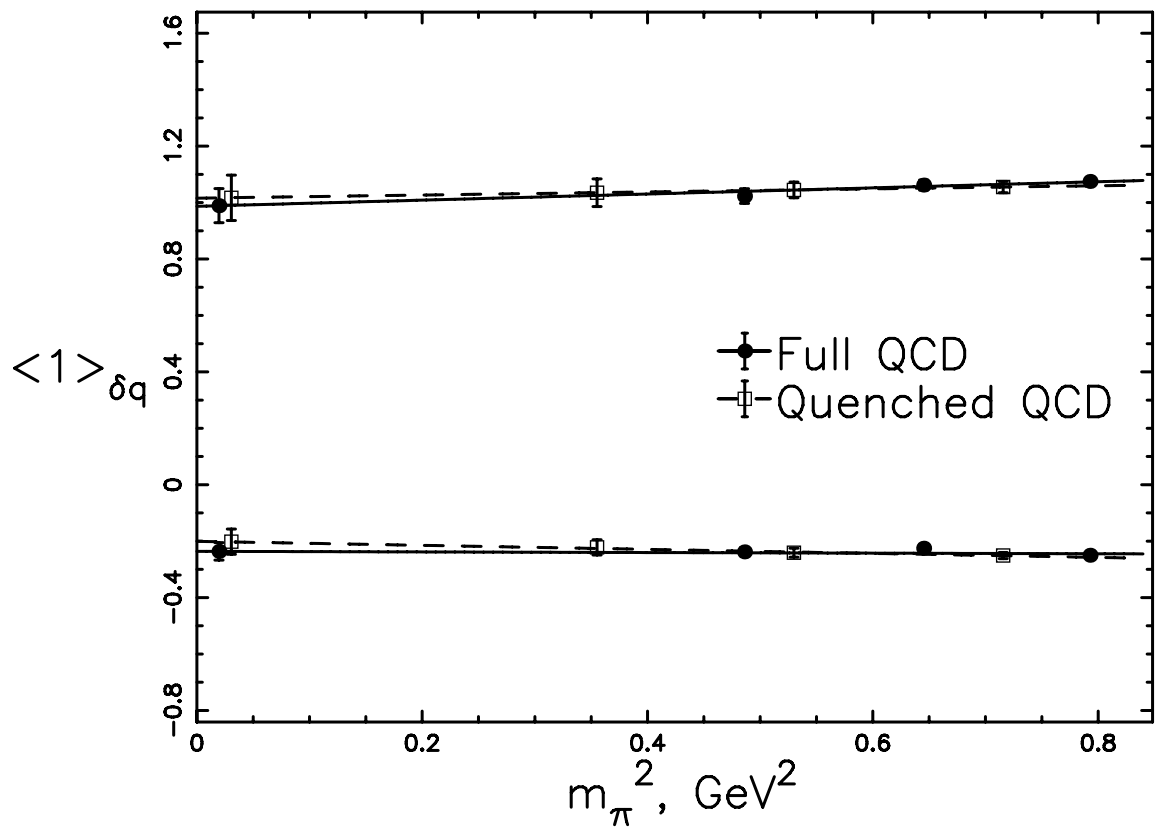

FIG. 19. Comparison of linear extrapolations of quenched and full QCD calculations of the tensor charge, $\langle 1\rangle_{\delta q}$, where the symbols are as in Fig. 16. 


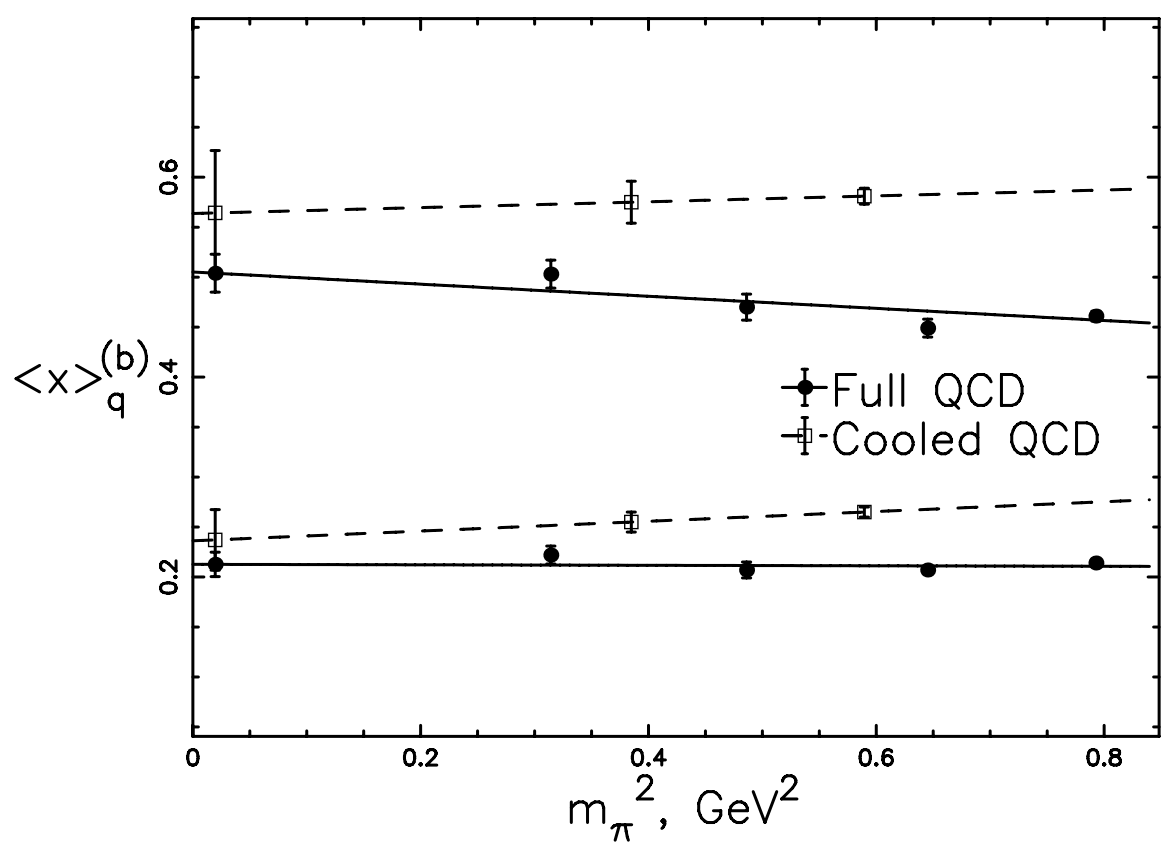

FIG. 20. Comparison of the quark momentum fraction $\langle x\rangle_{q}^{(b)}$ calculated in full QCD and using configurations cooled to eliminate essentially all contributions except those of instantons. Solid symbols connected by solid curves denote full QCD and open symbols connected by dashed curves denote results after cooling. The upper and lower curves correspond to up and down quarks respectively.

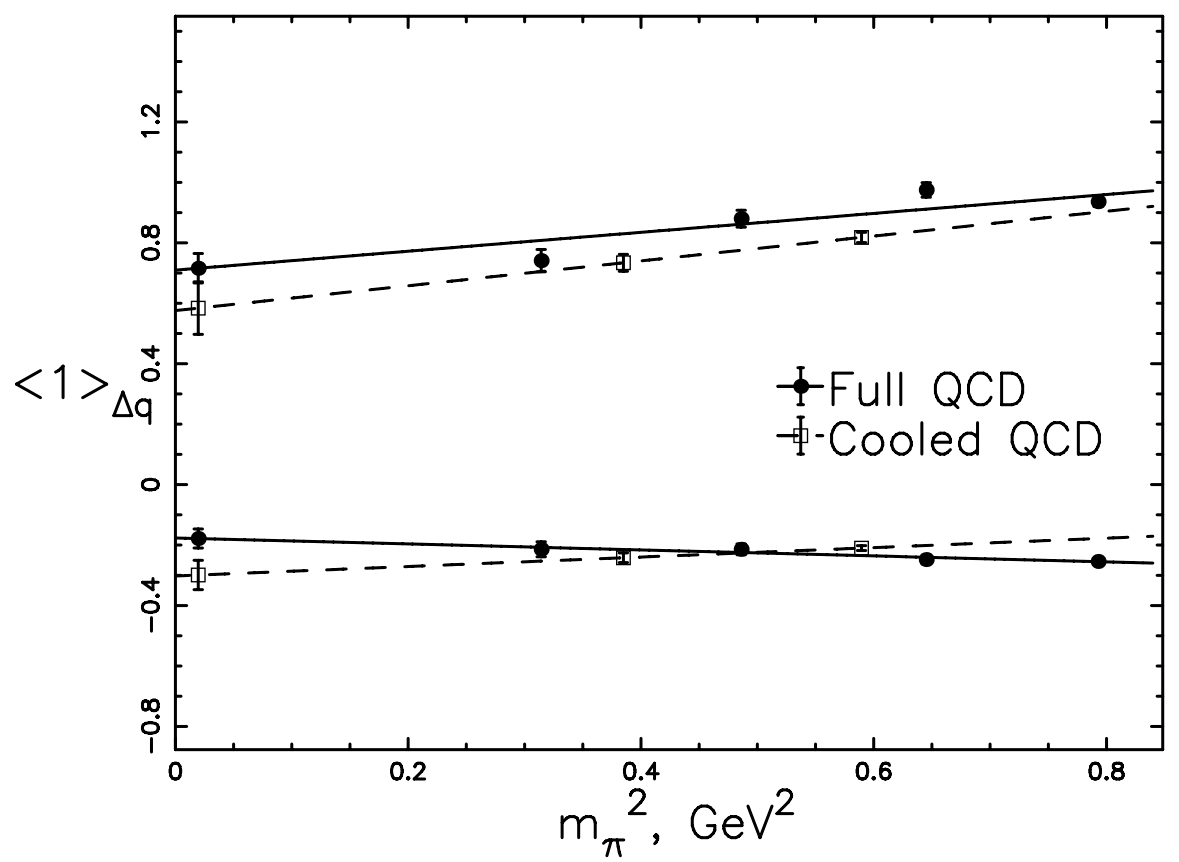

FIG. 21. Comparison of the axial charge $\langle 1\rangle_{\Delta q}$ calculated in full QCD and using cooled configurations, as in Fig. 20. 


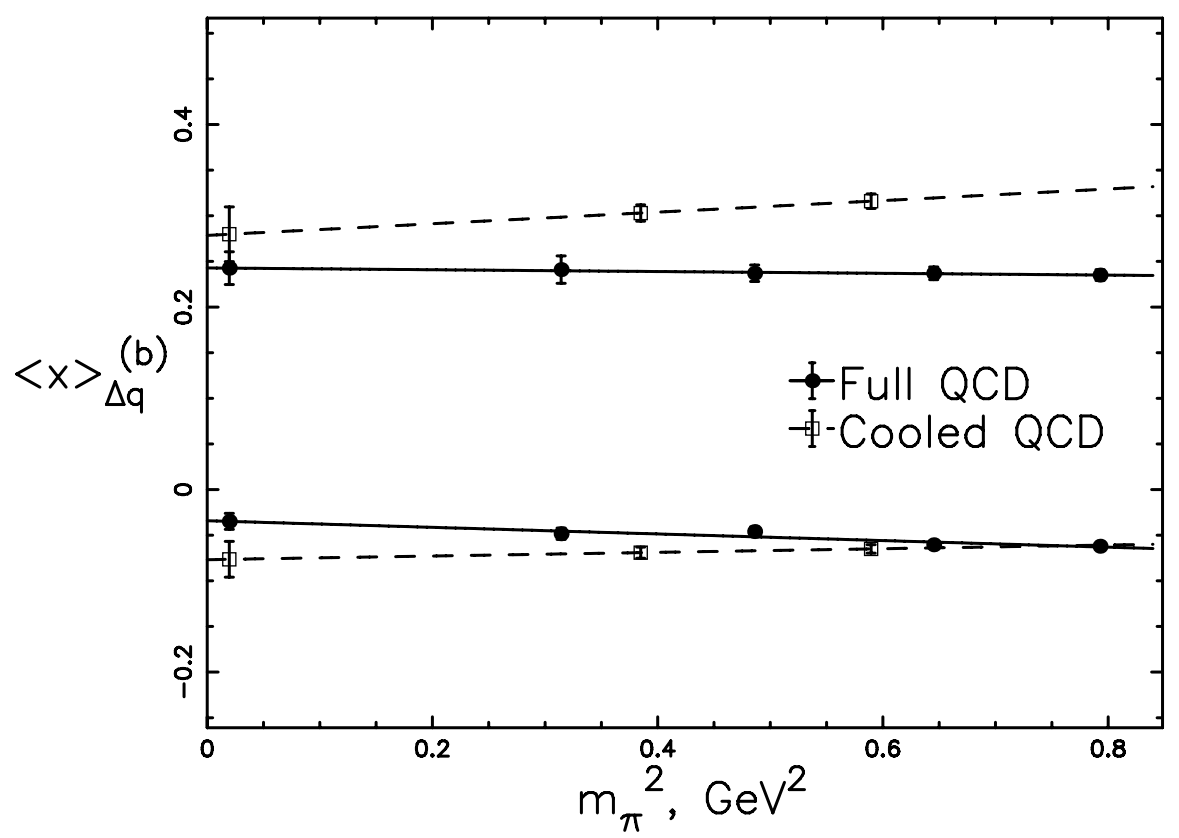

FIG. 22. Comparison of the first moment of the quark spin distribution, $\langle x\rangle_{\Delta q}^{(b)}$, calculated in full QCD and using cooled configurations, as in Fig. 20.

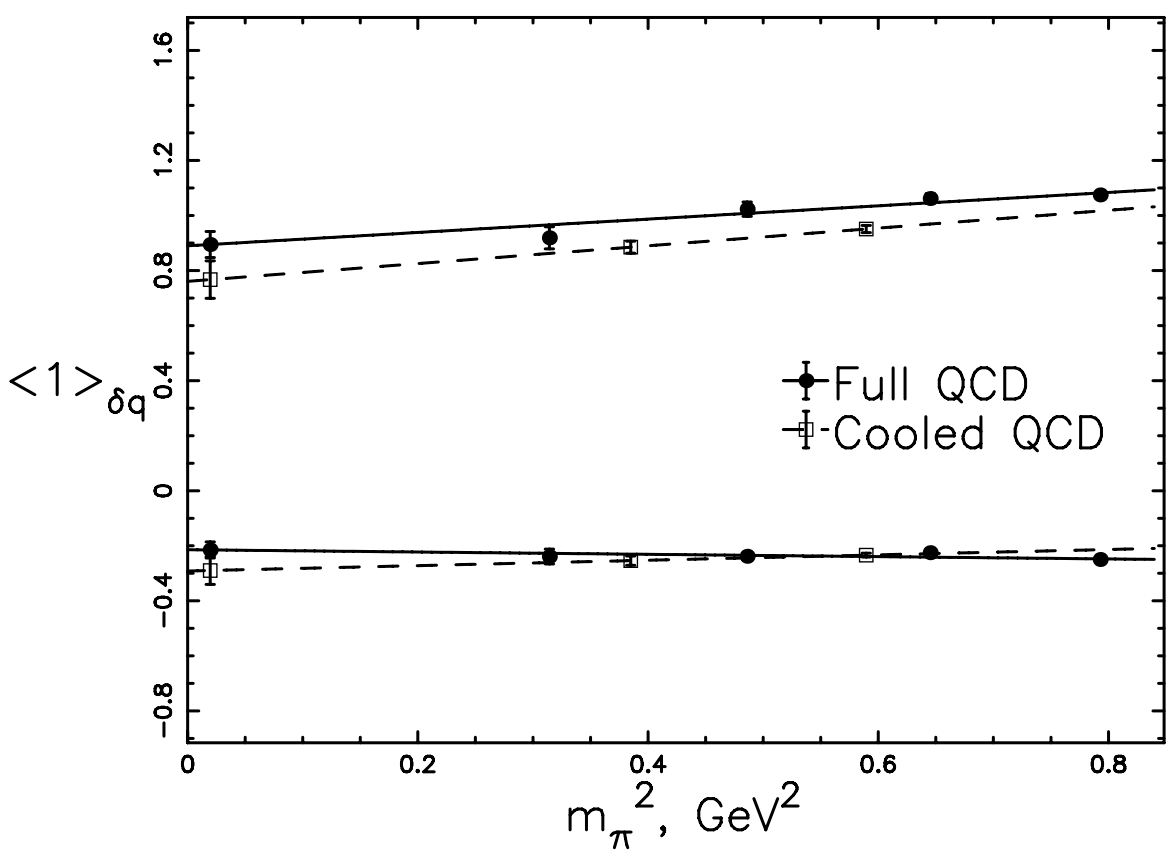

FIG. 23. Comparison of the tensor charge $\langle 1\rangle_{\delta q}$ calculated in full QCD and using cooled configurations, as in Fig. 20 . 


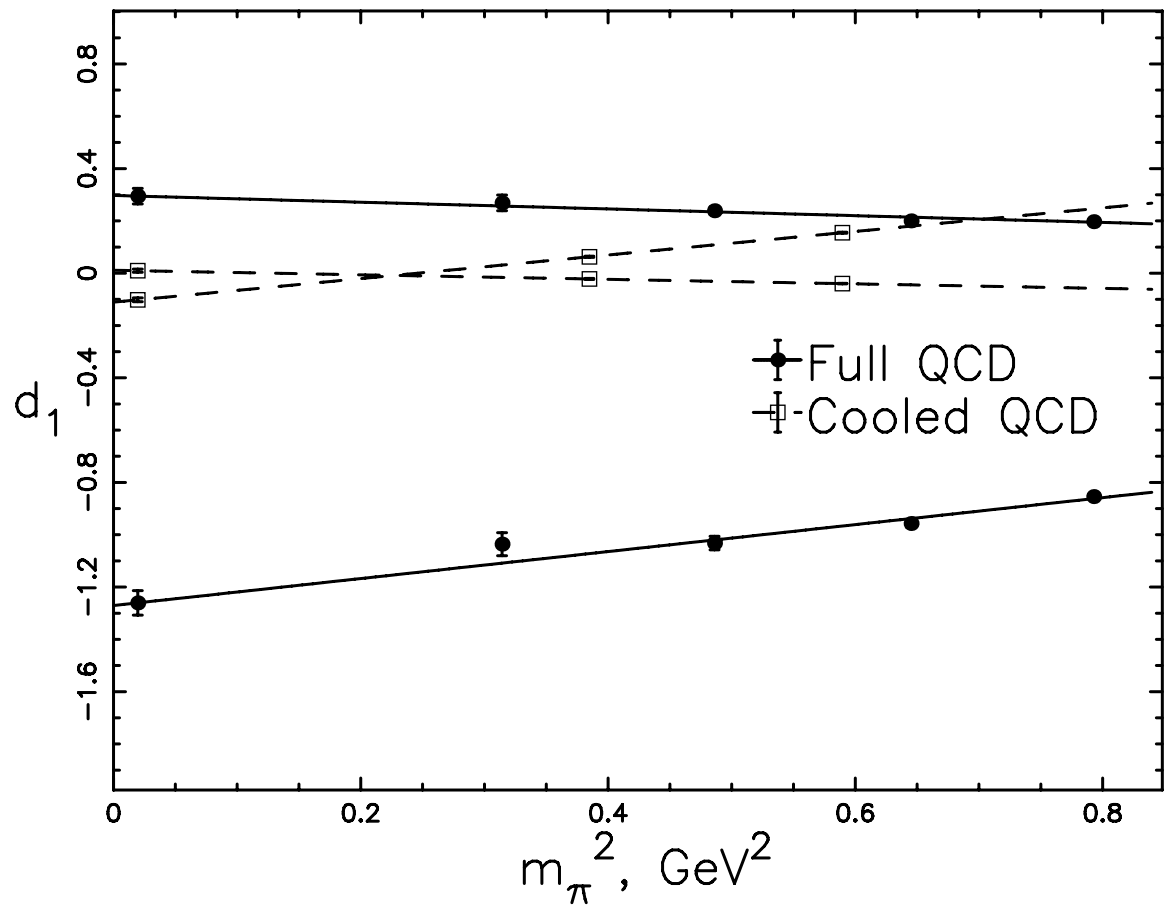

FIG. 24. Comparison of the twist-three operator $d_{1}$ from Eq. 8 calculated in full QCD and using cooled configurations, as in Fig. 20 .

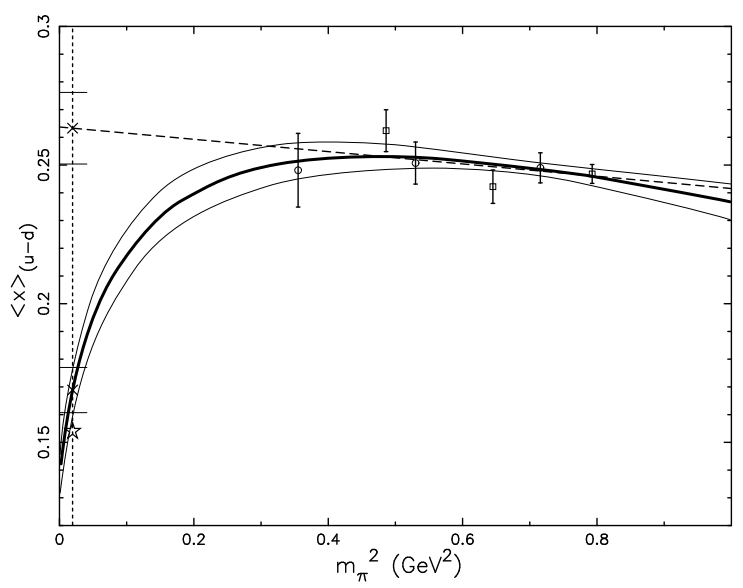

FIG. 25. Chiral extrapolation of the momentum fraction $\langle x\rangle_{u-d}$ using Eq. 21. Full QCD and quenched data calculated in this present work are denoted by squares and circles respectively, and the phenomenological result is indicated by the star. The least-squares fit and jackknife error bars are denoted by the heavy solid line and surrounding light lines. 


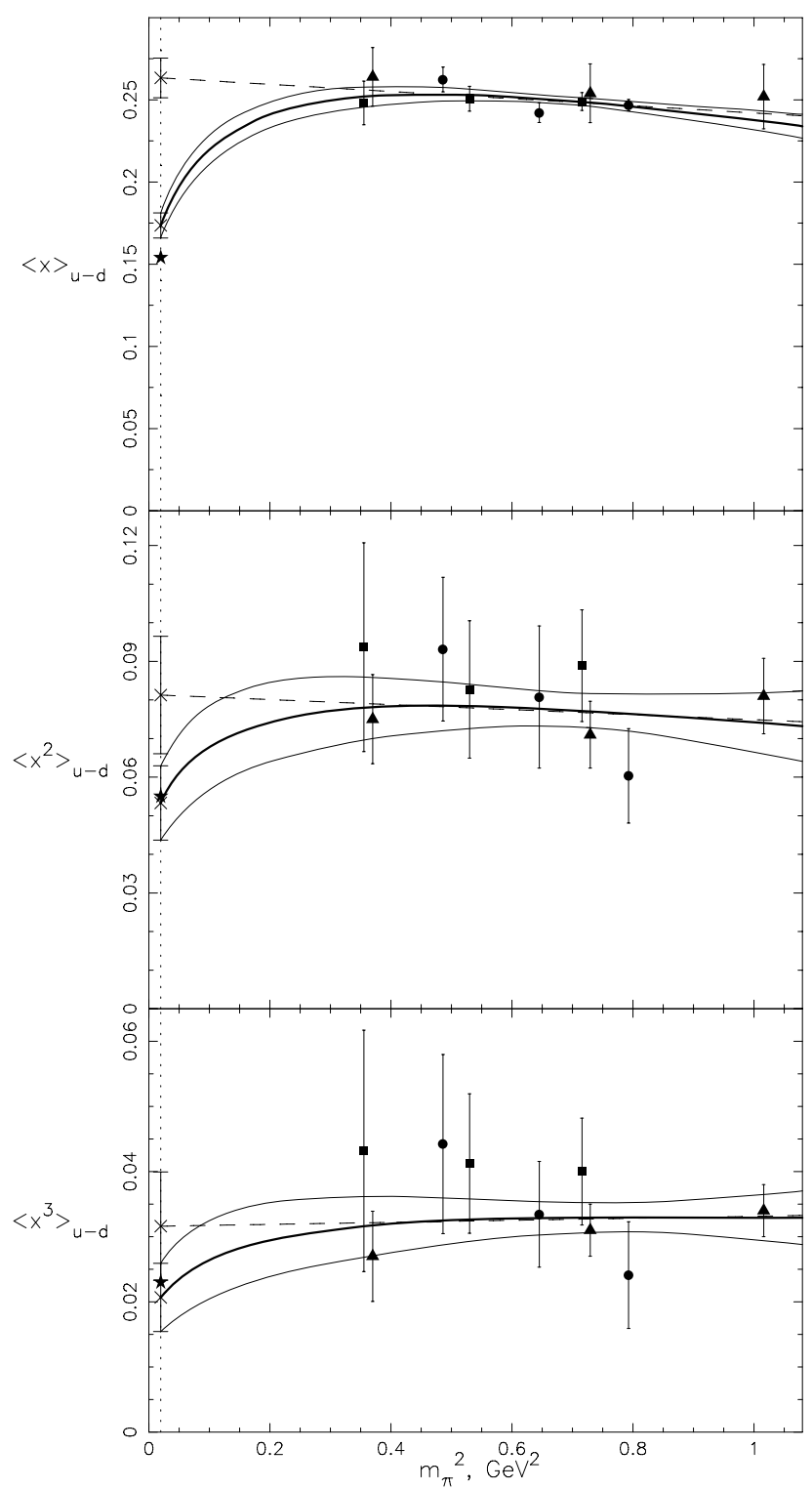

FIG. 26. Chiral extrapolation of the first three moments of the proton quark distribution, $\left\langle x^{n}\right\rangle_{u-d}$, using Eq. 21. Full QCD and quenched data calculated in this present work are denoted by diamonds and squares respectively, QCDSF quenched data [10] are denoted by triangles, and the phenomenological results are indicated by stars. The least-squares fit and jackknife error bars are denoted by the heavy solid line and surrounding light lines. 